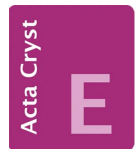

CRYSTALLOGRAPHIC COMMUNICATIONS

ISSN 2056-9890

\section{Crystal structures of two PCN pincer iridium complexes and one PCP pincer carbodiphosphorane iridium intermediate: substitution of one phosphine moiety of a carbodiphosphorane by an organic azide}

\author{
Gabriel Julian Partl,* Felix Nussbaumer, Walter Schuh, Holger Kopacka, Klaus \\ Wurst and Paul Peringer
}

Received 21 November 2018 Accepted 13 December 2018

Edited by $\mathrm{H}$. Stoeckli-Evans, University of Neuchâtel, Switzerland

Keywords: crystal structure; carbodiphosphorane; iridium; organic azide; reductive elimination; PCP pincer; non-innocent behaviour; PCN pincer.

CCDC references: 1880135; 1880134; 1880133

Supporting information: this article has supporting information at journals.iucr.org/e

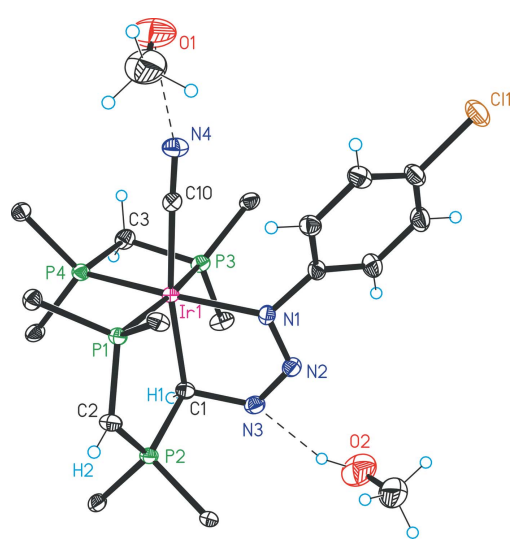

OPEN $\odot$ ACCESS
Institute of General, Inorganic and Theoretical Chemistry, University of Innsbruck, A-6020 Innsbruck, Austria. *Correspondence e-mail: gabriel.partl@uibk.ac.at

The structure of $\left[\operatorname{Ir}\left\{\left(4-\mathrm{Cl}-\mathrm{C}_{6} \mathrm{H}_{4} \mathrm{~N}_{3}\right) \mathrm{C}(\mathrm{dppm})-\kappa^{3} P, C, N\right\}\left(\mathrm{dppm}-\kappa^{2} P, P^{\prime}\right)\right] \mathrm{Cl} \cdot-$ $1.5 \mathrm{CH}_{2} \mathrm{Cl}_{2} \cdot 0.5 \mathrm{C}_{7} \mathrm{H}_{8}\left(\mathrm{C}_{57} \mathrm{H}_{48} \mathrm{Cl}_{2} \mathrm{IrN}_{3} \mathrm{P}_{4} \cdot 1.5 \mathrm{CH}_{2} \mathrm{Cl}_{2} \cdot 0.5 \mathrm{C}_{7} \mathrm{H}_{8}\right)$ (2), dppm = bis (diphenylphosphino)methane \{systematic name: [7-(4-chlorophenyl)-1,1,3,3-tetraphenyl-5,6,7-triaza- $\kappa N^{7}-1,3 \lambda^{4}$-diphospha- $\kappa P^{1}$-hepta-4,6-dien-4-yl][methylenebis(diphenylphosphine) $\left.-\kappa^{2} P, P^{\prime}\right] \operatorname{iridium}(\mathrm{I}) \quad$ chloride-dichloromethane-toluene $(2 / 3 / 1)\}$, resulting from the reaction of $\left[\mathrm{IrClH}\left\{\mathrm{C}(\mathrm{dppm})_{2}-\kappa^{3} P, C, P\right)(\mathrm{MeCN})\right] \mathrm{Cl}$ (1a) with 1-azido-4-chlorobenzene, shows a monocationic five-coordinate $\operatorname{Ir}^{\mathrm{I}}$ complex with a distorted trigonal-bipyramidal geometry. In $\mathbf{2}$, the iridium centre is coordinated by the neutral triazeneylidenephosphorane (4-Cl- $\mathrm{C}_{6} \mathrm{H}_{4} \mathrm{~N}_{3}$ )$\mathrm{C}(\mathrm{dppm})$ acting as a PCN pincer ligand, and a chelating dppm unit. The structure of the coordination compound $\left[\mathrm{IrCl}(\mathrm{CN}) \mathrm{H}\left(\mathrm{C}(\mathrm{dppm})_{2}-\kappa^{3} P, C, P\right)\right] \cdot-$ $\mathrm{CH}_{3} \mathrm{CN}, \quad\left(\mathrm{C}_{52} \mathrm{H}_{45} \mathrm{ClIrNP}_{4} \cdot \mathrm{CH}_{3} \mathrm{CN}\right)$ (1b) [systematic name: chloridocyanidohydrido $\left(1,1,3,3,5,5,7,7\right.$-octaphenyl-1,3 $\lambda^{5}, 5 \lambda^{4}, 7$-tetraphospha- $\kappa^{2} P^{1}, P^{7}$ hept-3-en-4-yl)iridium(III) acetonitrile monosolvate], prepared from $\mathbf{1 a}$ and $\mathrm{KCN}$, reveals an octahedral $\mathrm{Ir}^{\mathrm{III}}$ central atom with a meridional PCP pincer carbodiphosphorane (CDP) ligand; the chloride ligand is located trans to the central carbon of the CDP functionality while the hydrido and cyanido ligands are situated trans to each other. The chiral coordination compound $[\operatorname{Ir}(\mathrm{CN})((4-$ $\left.\left.\left.\mathrm{Cl}-\mathrm{C}_{6} \mathrm{H}_{4} \mathrm{~N}_{3}\right) \mathrm{CH}\left(\mathrm{CH}\left(\mathrm{P}(\mathrm{Ph})_{2}\right)_{2}\right)-\kappa^{3} P, C, N\right)\left(\mathrm{dppm}-\kappa^{2} P, P^{\prime}\right)\right] \cdot 2 \mathrm{CH}_{3} \mathrm{OH}, \quad\left(\mathrm{C}_{58} \mathrm{H}_{48} \mathrm{ClIr}-\right.$ $\mathrm{N}_{4} \mathrm{P}_{4} \cdot 2 \mathrm{CH}_{3} \mathrm{OH}$ ) (3) (systematic name: \{4-[3-(4-chlorophenyl)triazenido- $\kappa N^{3}$ ]1,1,3,3-tetraphenyl-1,3$\lambda^{5}$-diphospha- $\kappa P^{1}$-but-2-en-4-yl\}cyanido[methylenebis(diphenylphosphine) $\left.-\kappa^{2} P, P^{\prime}\right]$ iridium(III) methanol disolvate), formed via prolonged reaction of 1-azido-4-chlorobenzene with $\mathbf{1 b}$, features a sixcoordinate $\mathrm{Ir}^{\mathrm{III}}$ central atom. The iridium centre is coordinated by the dianionic facial $\mathrm{PCN}$ pincer ligand $\left[\left(4-\mathrm{Cl}-\mathrm{C}_{6} \mathrm{H}_{4} \mathrm{~N}_{3}\right) \mathrm{CH}\left(\mathrm{CH}\left(\mathrm{P}\left(\mathrm{Ph}_{2}\right)_{2}\right)_{2}\right)\right]$, a cyanido ligand trans to the central carbon of the $\mathrm{PCN}$ pincer ligand and a chelating dppm unit. Complex 2 exhibits a 2:1 positional disorder of the $\mathrm{Cl}^{-}$anion. The $\mathrm{CH}_{2} \mathrm{Cl}_{2}$ and $\mathrm{C}_{7} \mathrm{H}_{8}$ solvent molecules show occupational disorder, with the toluene molecule exhibiting additional 1:1 positional disorder with some nearly overlying carbon atoms.

\section{Chemical context}

Carbodiphosphoranes (CDPs), also termed double ylides, consist of two tertiary phosphines connected to a central divalent carbon(0) atom. The $\mathrm{P}-\mathrm{C}$ bonds are best described as donor-acceptor interactions (Petz \& Frenking, 2010). Most of the chemistry associated with CDPs concerns compounds with Lewis acids. Since the central CDP carbon possesses two 
lone electron pairs, it is therefore able to interact with either one or two Lewis acids (Chauvin \& Canac, 2010; Petz \& Frenking, 2010). Reactions involving the cleavage of the $\mathrm{P}-\mathrm{C}$ bonds of the CDP functionality are less common in contrast to phosphorus ylides (Petz \& Frenking, 2010; Kolodiazhnyi, 1999). We hereby report the non-innocent reactivity (Poverenov \& Milstein, 2013) of a PCP pincer ligand, whose central carbon is part of a CDP functionality, with an organic azide in the coordination sphere of iridium.

Treatment of the $\mathrm{Ir}^{\mathrm{III}}$ PCP pincer CDP complex $\left[\mathrm{Ir}(\mathrm{Cl})(\mathrm{H})\left(\mathrm{C}(\mathrm{dppm})_{2}-\kappa^{3} P, C, P\right)(\mathrm{MeCN})\right] \mathrm{Cl} \quad$ (1a) (SchlappHackl et al., 2018) with 1-azido-4-chlorobenzene affords the $\operatorname{Ir}^{\mathrm{I}} \quad$ complex $\quad\left[\operatorname{Ir}\left(\left(4-\mathrm{Cl}_{-} \mathrm{C}_{6} \mathrm{H}_{4} \mathrm{~N}_{3}\right) \mathrm{C}(\mathrm{dppm})-\kappa^{3} P, C, N\right)(\mathrm{dppm}-\right.$ $\left.\left.\kappa^{2} P, P^{\prime}\right)\right] \mathrm{Cl}(2)$. The reaction implies the substitution of one phosphine moiety of the PCP pincer ligand $\mathrm{C}(\mathrm{dppm})_{2}$ for the organic azide, thus producing the triazeneylidenephosphorane (4- $\left.\mathrm{Cl}-\mathrm{C}_{6} \mathrm{H}_{4} \mathrm{~N}_{3}\right) \mathrm{C}(\mathrm{dppm})$, which acts as a $\mathrm{PCN}$ pincer ligand in 2. The phosphine displaced from the CDP functionality ends up in the coordination sphere of iridium and becomes part of a four-membered dppm chelate ring (see scheme).

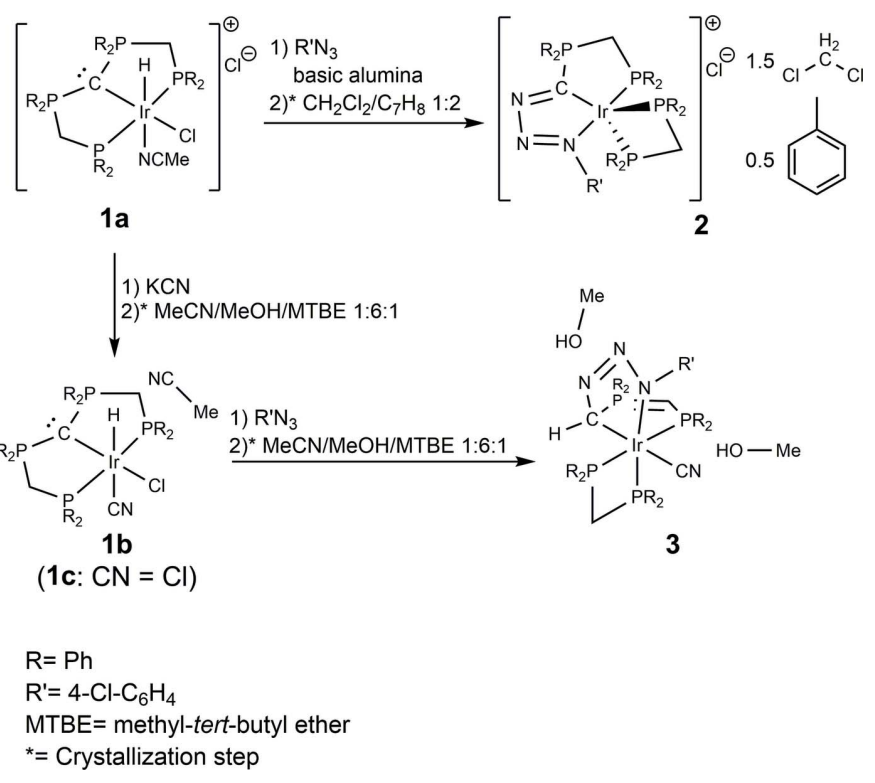

We believe that the reaction is initiated by an interaction of the electrophilic organic azide with the central CDP carbon of the PCP ligand, which disposes of one lone electron pair (Petz \& Frenking, 2010). In a related reaction, N-heterocyclic carbenes (NHCs) have been reported to form end-on adducts with organic azides to form triazenes (Khramov \& Bielawski, 2005). The interaction of the CDP with the organic azide results in the formation of a double bond between the central carbon and the terminal nitrogen of the organic azide and is associated with the cleavage of one $\mathrm{P}-\mathrm{C}$ bond of the CDP functionality, while the carbon-iridium bond remains intact. At this stage, a deeply coloured and presumably five-coordinate $\mathrm{Ir}^{\mathrm{I}}$ intermediate was detected by monitoring the reaction via ${ }^{31} \mathrm{P}$-NMR spectroscopy. This intermediate features the triazenylidenephosphorane ligand $\left(4-\mathrm{Cl}-\mathrm{C}_{6} \mathrm{H}_{4} \mathrm{~N}_{3}\right) \mathrm{C}(\mathrm{dppm})$ and a monodentate dppm unit. The absence of a hydrido ligand is attributed to an antecedent reductive elimination of hydrochloric acid, which, according to NMR spectroscopic results, is absorbed by the CDP carbon of the starting complex 1a. This carbon atom turns out to be the strongest base of the system, apparently more basic than the nitrogen atoms and the central carbon of the PCN pincer ligand of 2 . Consequently, only $50 \%$ of the educt is converted to $\mathbf{2}$. However, an almost quantitative and fast conversion into $\mathbf{2}$ was achieved upon addition of basic alumina. The formation of $\mathbf{2}$ is finalized via the dissociation of a chlorido ligand and the coordination of the displaced phosphine functionality to the Ir centre.

Treatment of 1a with $\mathrm{KCN}$ affords $[\operatorname{Ir}(\mathrm{Cl})(\mathrm{CN})(\mathrm{H})$ $\left.\left(\left(\mathrm{C}(\mathrm{dppm})_{2}\right)-\kappa^{3} P, C, P\right)\right](\mathbf{1 b})$, which reacts very slowly (over the course of weeks at room temperature) with 1-azido-4chlorobenzene to the six-coordinate complex $[\operatorname{Ir}(\mathrm{CN})((4-\mathrm{Cl}-$ $\left.\left.\left.\mathrm{C}_{6} \mathrm{H}_{4} \mathrm{~N}_{3}\right) \mathrm{CH}\left(\mathrm{CH}\left(\mathrm{PPh}_{2}\right)_{2}\right)-\kappa^{3} P, C, N\right)\left(\mathrm{dppm}-\kappa^{2} P, P^{\prime}\right)\right] \quad$ (3). Related to the formation of $\mathbf{2}$, the organic azide substitutes one phosphine of the CDP functionality.

At first sight, the resulting PCN pincer ligand of $\mathbf{3}$ looks like a tautomer of the PCN pincer ligand of 2: while in the PCN pincer of $\mathbf{3}$, one proton is attached to $\mathrm{C} 1$ and $\mathrm{C} 2$ respectively, the $\mathrm{PCN}$ pincer of $\mathbf{2}$ carries two protons at $\mathrm{C} 2$ and none at $\mathrm{C} 1$. In contrast to the neutral ligand of $\mathbf{2}$, the PCN ligand in $\mathbf{3}$ carries a double negative charge, deduced as follows: first, in view of coordination number $6, \mathbf{3}$ constitutes an $\mathrm{Ir}^{\mathrm{III}}$ complex. Second, the coordination compound $\mathbf{3}$ carries no charge. Third, the cyanido ligand contributes a -1 charge, and the iridium central atom a +3 charge. Since the dppm ligand is neutral, the charge of the PCN pincer ligand can be calculated to be -2 . We suspect that the pathway of the reaction is similar to the formation of $\mathbf{2}$, except that the cyanido ligand permanently stays in the coordination sphere of iridium. The coordination of the displaced phosphine functionality to the $\operatorname{Ir}^{\mathrm{I}}$ centre is thought to induce a two-electron transfer from iridium to the PCN ligand related to an oxidative addition reaction, and to be followed by the transfer of one proton from $\mathrm{C} 2$ to $\mathrm{C} 1$.

\section{Structural commentary}

The structures of compounds $\mathbf{2}, \mathbf{1 b}$ and $\mathbf{3}$ are given in Figs. 1, 2 and 3, respectively. Selected bond lengths and angles for all three compounds are given in Table 1.

The structure of 2 (Fig. 1) features a cationic five-coordinate iridium(I) complex with a chloride counter-ion. The asymmetric unit additionally contains 1.5 molecules of dichloromethane and half a molecule of toluene. The iridium centre is coordinated by a PCN pincer ligand and a chelating dppm; its coordination sphere displays a distorted trigonal-bipyramidal geometry, in which the PCN pincer occupies one axial (C1) and two equatorial (P1 and N1) positions. The donor atoms of the chelating dppm are found in the remaining axial (P3) and equatorial (P4) positions. Major distortions are apparent from the angles $\mathrm{P} 4-\mathrm{Ir} 1-\mathrm{P} 3 \quad\left[70.69(5)^{\circ}\right]$ and $\mathrm{N} 1-\mathrm{Ir} 1-\mathrm{P} 1$ $\left[140.4(1)^{\circ}\right]$, which reflect the ring strain of both the fourmembered dppm chelate ring and the PCN pincer ligand. The 


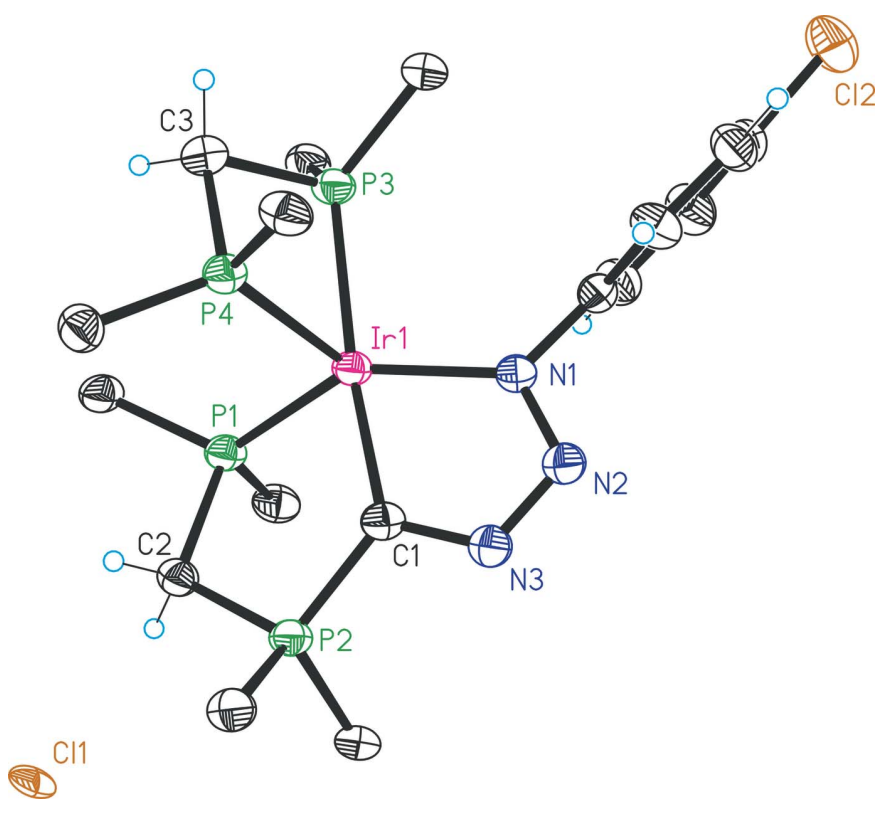

Figure 1

Structure of $\mathbf{2}$ with displacement ellipsoids drawn at the $30 \%$ probability level. For clarity, only the ipso carbon atoms of the phenyl groups are shown, and solvent molecules have been omitted.

angles around the ylidic carbon $\mathrm{C} 1$ cover a range of 115.9 (4) to $124.5(3)^{\circ}$, with a sum total of $359^{\circ}$. The $\mathrm{C} 1=\mathrm{N} 3$ bond exhibits an increased length [1.346 (7) A] relative to typical $\mathrm{C}=\mathrm{N}$ double bonds $(1.29 \AA)$, resulting in a formal bond order (BO) of 1.7. With a bond length of 1.308 (6) $\AA$, the N2-N3 bond's $\mathrm{BO}$ is 1.7 as well; the $\mathrm{N} 1-\mathrm{N} 2$ distance amounts to 1.354 (6) $\AA$ (BO 1.5). In reported adducts of NHCs with organic azides, the $\mathrm{C}-\mathrm{N} 3$ (numbering as in the free azides) bond lengths are similar to 2 , whereas $\mathrm{N} 1-\mathrm{N} 2$ separations are shorter ( $c a 1.27 \AA$ ) and the $\mathrm{N} 2$ - N3 distances are longer ( $c a$ $1.35 \AA$ ) compared to the corresponding bond lengths in 2

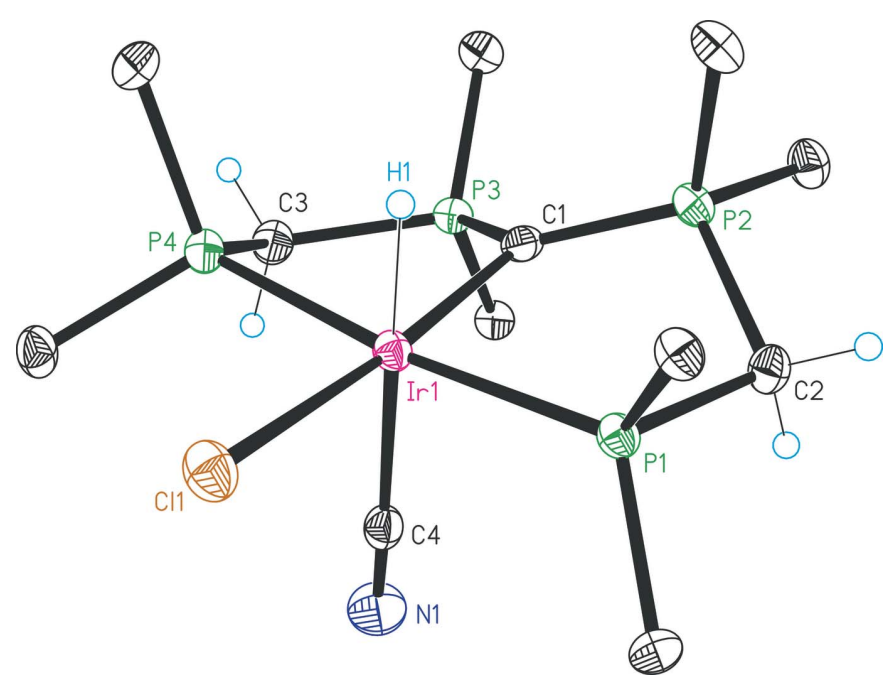

Figure 2

Structure of $\mathbf{1 b}$ with displacement ellipsoids drawn at the $30 \%$ probability level. Only the ipso carbon atoms of the phenyl groups are shown for clarity, and solvent molecules have been omitted.
(Khramov \& Bielawski, 2005). These differences are presumably due to the coordination of N3 to the Ir centre. Organic azides themselves exhibit a short N2 $-\mathrm{N} 3$ bond [e.g. 1.1322 (2) $\AA$, BO 2.5, for 2,4,6-trichlorphenylazide], whereas the $\mathrm{N} 1-\mathrm{N} 2$ bond is distinctly longer [1.252 (2) $\AA$, BO 1.9; Takayama et al., 2010].

The structure of $\mathbf{1 b}$ (Fig. 2) displays an octahedral iridium(III) coordination compound with a meridional $\mathrm{C}(\mathrm{dppm})_{2}$ PCP pincer ligand and one chlorido ligand situated trans to the central CDP carbon atom. The remaining sites are occupied by the hydrido and cyanido ligands positioned trans to each other. The structure is closely related to that of $\left.\left[\operatorname{Ir}(\mathrm{Cl})_{2}(\mathrm{H})\left(\mathrm{C}(\mathrm{dppm})_{2}\right)-\kappa^{3} P, C, P\right)\right]$ (1c) (Partl et al., 2018), which contains one chlorido ligand instead of the cyanido ligand trans to the hydrido ligand. The introduction of the cyanido ligand results in a markedly shorter Ir1-Cl1 bond trans to the CDP carbon $[2.445$ (1) $\AA$ compared to 2.5157 (14) $\AA$ for 1c], whereas the $\mathrm{Ir} 1-\mathrm{C} 1$ separation becomes longer [2.128 (4) $\AA$ compared to 2.101 (5) $\AA$ ] . Ir $-\mathrm{P}$ distances are marginally affected (Table 1 ).

The structure of $\mathbf{3}$ (Fig. 3) shows a six-coordinate iridiu$\mathrm{m}$ (III) coordination compound with the $\mathrm{PCN}$ pincer ligand $\left[\left(4-\mathrm{Cl}-\mathrm{C}_{6} \mathrm{H}_{4} \mathrm{~N}_{3}\right) \mathrm{CH}\left(\mathrm{CH}\left(\mathrm{PPh}_{2}\right)_{2}\right)\right]$ in a facial mode, a bidentate dppm and a cyanido ligand trans to the central PCN carbon. Two molecules of $\mathrm{MeOH}$ are connected to atoms N3 and N4 via hydrogen bonds. Distortions of the octahedral geometry are evident from the angles $\mathrm{P} 3-\mathrm{Ir} 1-\mathrm{P} 4$ and $\mathrm{N} 1-\mathrm{Ir} 1-\mathrm{C} 1$, amounting to $72.19(2)^{\circ}$ and $75.31(9)^{\circ}$, respectively. The environment of the chiral carbon $\mathrm{C} 1$ is distorted tetrahedral according to the angles N3-C1- Ir1 [109.8 (2) $\left.{ }^{\circ}\right], \mathrm{N} 3-\mathrm{C} 1-$

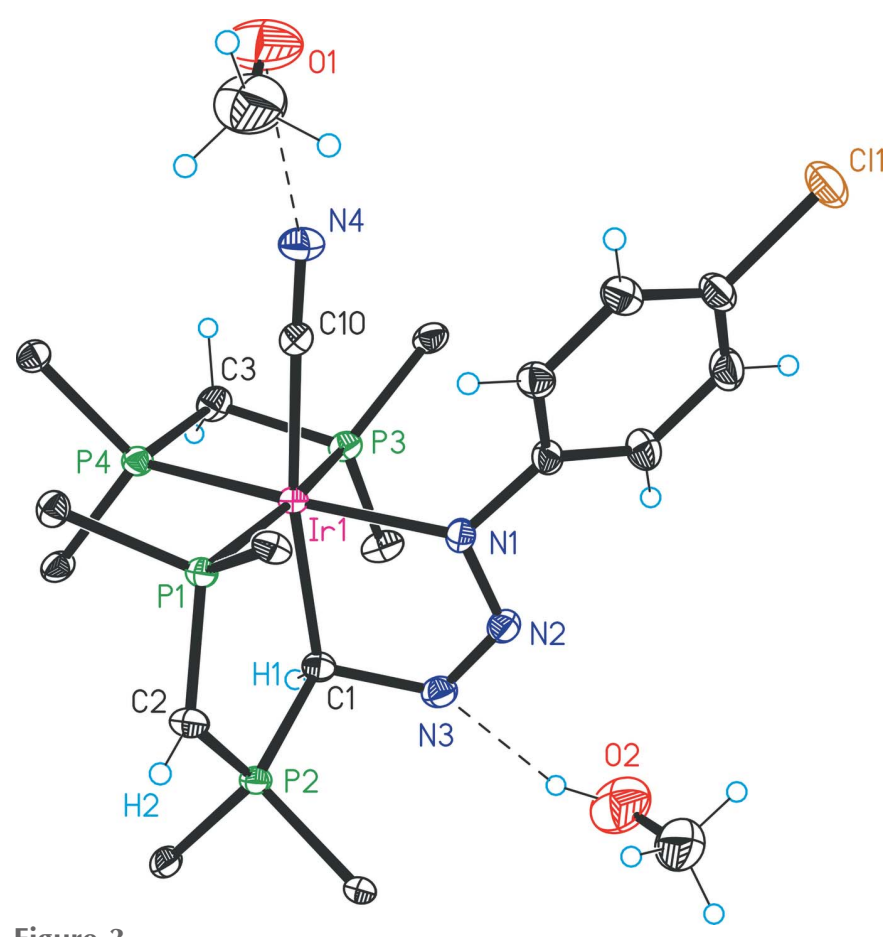

Figure 3

Structure of $\mathbf{3}$ with displacement ellipsoids drawn at the $30 \%$ probability level. Only the ipso carbon atoms of the phenyl groups are shown for clarity. 
Table 1

Selected bond lengths $(\AA)$ and angles $\left(^{\circ}\right)$ for $\mathbf{1 b}, \mathbf{2}$ and $\mathbf{3}$.

\begin{tabular}{|c|c|c|c|c|c|}
\hline $\mathbf{1 b}$ & & 2 & & 3 & \\
\hline \multirow[t]{2}{*}{$\mathrm{Ir} 1-\mathrm{C} 1$} & \multirow[t]{2}{*}{$2.128(4)$} & $\mathrm{Ir} 1-\mathrm{C} 1$ & $1.996(5)$ & $\mathrm{Ir} 1-\mathrm{C} 1$ & $2.127(3)$ \\
\hline & & Ir1-N1 & $1.999(5)$ & $\mathrm{Ir} 1-\mathrm{N} 1$ & $2.109(2)$ \\
\hline Ir1-P1 & $2.302(1)$ & Ir1-P1 & $2.2788(14)$ & $\mathrm{Ir} 1-\mathrm{P} 1$ & $2.3595(6)$ \\
\hline $\mathrm{Ir} 1-\mathrm{Cl} 1$ & $2.445(1)$ & Ir1-P3 & $2.3748(14)$ & Ir1-P3 & $2.3584(7)$ \\
\hline $\mathrm{Ir} 1-\mathrm{C} 4$ & $2.0815(5)$ & Ir1-P4 & $2.2662(14)$ & Ir1-P4 & $2.3304(6)$ \\
\hline $\mathrm{Ir} 1-\mathrm{H} 1$ & $1.51(4)$ & & & Ir1-C10 & $2.026(2)$ \\
\hline $\mathrm{P} 2-\mathrm{C} 1$ & $1.689(4)$ & $\mathrm{P} 2-\mathrm{C} 1$ & $1.776(5)$ & $\mathrm{P} 2-\mathrm{C} 1$ & $1.843(2)$ \\
\hline & & $\mathrm{N} 2-\mathrm{N} 3$ & $1.308(6)$ & $\mathrm{N} 2-\mathrm{N} 3$ & $1.259(3)$ \\
\hline $\mathrm{C} 4-\mathrm{Ir} 1-\mathrm{P} 1$ & $94.22(12)$ & $\mathrm{C} 1-\mathrm{Ir} 1-\mathrm{P} 1$ & $85.35(15)$ & $\mathrm{C} 1-\mathrm{Ir} 1-\mathrm{P} 1$ & $87.23(7)$ \\
\hline $\mathrm{C} 1-\mathrm{Ir} 1-\mathrm{P} 1$ & 89.10 (12) & $\mathrm{N} 1-\mathrm{Ir} 1-\mathrm{P} 1$ & $140.40(13)$ & $\mathrm{N} 1-\mathrm{Ir} 1-\mathrm{P} 1$ & $96.69(6)$ \\
\hline $\mathrm{P} 4-\mathrm{Ir} 1-\mathrm{P} 1$ & $173.16(4)$ & $\mathrm{P} 4-\mathrm{Ir} 1-\mathrm{P} 1$ & $95.59(5)$ & $\mathrm{P} 4-\mathrm{Ir} 1-\mathrm{P} 1$ & $98.11(2)$ \\
\hline $\mathrm{C} 4-\mathrm{Ir} 1-\mathrm{P} 4$ & 89.08 (12) & $\mathrm{C} 1-\mathrm{Ir} 1-\mathrm{P} 3$ & $173.04(16)$ & $\mathrm{P} 3-\mathrm{Ir} 1-\mathrm{P} 1$ & $169.15(2)$ \\
\hline $\mathrm{C} 4-\mathrm{Ir} 1-\mathrm{C} 1$ & 89.27 (17) & $\mathrm{C} 1-\mathrm{Ir} 1-\mathrm{N} 1$ & $73.7(2)$ & $\mathrm{C} 10-\mathrm{Ir} 1-\mathrm{P} 4$ & $89.88(7)$ \\
\hline $\mathrm{C} 4-\mathrm{Ir} 1-\mathrm{H} 1$ & $176.6(13)$ & & & $\mathrm{N} 1-\mathrm{Ir} 1-\mathrm{P} 4$ & $164.45(6)$ \\
\hline & & & & $\mathrm{C} 1-\mathrm{Ir} 1-\mathrm{P} 4$ & $100.63(7)$ \\
\hline & & & & $\mathrm{C} 10-\mathrm{Ir} 1-\mathrm{N} 1$ & $94.15(9)$ \\
\hline & & & & $\mathrm{C} 10-\mathrm{Ir} 1-\mathrm{C} 1$ & $169.38(9)$ \\
\hline & & & & $\mathrm{N} 1-\mathrm{Ir} 1-\mathrm{C} 1$ & $75.31(9)$ \\
\hline
\end{tabular}

P2 [104.9 (2) $)^{\circ}$ and P2-C1 - Ir1 [116.7 (1) $\left.{ }^{\circ}\right]$. The deprotonated dppm part of the PCN pincer ligand features delocalization over both $\mathrm{P}-\mathrm{C}$ bonds [P1 $-\mathrm{C} 21.727$ (3) and $\mathrm{P} 2-\mathrm{C} 2$ 1.688 (3) $\AA$, corresponding to a $\mathrm{BO}$ of $c a 1.5$ each). The $\mathrm{C} 1-$ $\mathrm{N} 3$ bond is rather long [1.504 (3) $\AA$, BO 0.8)] and appears to be in the range of protonated alkylamines, (Ishida, 2000) whereas the $\mathrm{N} 2=\mathrm{N} 3$ distance approximately corresponds to an $\mathrm{N}=\mathrm{N}$ double bond [1.259 (3) $\AA$, BO 1.9]. The $\mathrm{N} 1-\mathrm{N} 2$ separation is found to be in between a single and a double bond [1.347 (3) ̊̊, BO 1.5].

\section{Supramolecular features}

In the crystal of $\mathbf{2}$, supramolecular features appear to revolve around the chloride anion (Table 2): Cl1 interacts with the methylene group of one dppm unit $(\mathrm{C} 2-\mathrm{H} 2 B \cdots \mathrm{Cl} 1=2.62 \AA)$ and to a proton of one dichloromethane molecule (C11$\mathrm{H} 11 B \cdots \mathrm{Cl} 1^{\mathrm{i}}=2.49 \AA$ ). It must be mentioned, however, that due to the positional disorder of both the chloride anion and the dichloromethane solvate units, these 'bond' lengths are an estimation and may not necessarily reflect any actual intermolecular interactions.

In the crystal of $\mathbf{1 b}$, the nitrogen atom of the acetonitrile solvate interacts with the methylene group of one dppm unit in a hydrogen-bond like manner $(\mathrm{C} 3-\mathrm{H} 3 B \cdots \mathrm{N} 2=2.58 \AA$; Table 3). Intermolecular halogen-hydrogen interactions are observed in two instances between phenyl protons and the chlorido ligand $(\mathrm{C} 102-\mathrm{H} 102 \cdots \mathrm{Cl} 1=2.66 \AA$ and $\mathrm{C} 112-$ $\mathrm{H} 112 \cdots \mathrm{Cl} 1^{\mathrm{i}}=2.77 \AA$; Table 3$)$.

In the crystal of $\mathbf{3}$, intermolecular features are restricted to solvate coordination (Table 4): both methanol units are
Table 2

Hydrogen-bond geometry $\left(\AA,^{\circ}\right)$ for 2 .

\begin{tabular}{lllll}
\hline$D-\mathrm{H} \cdots A$ & $D-\mathrm{H}$ & $\mathrm{H} \cdots A$ & $D \cdots A$ & $D-\mathrm{H} \cdots A$ \\
\hline $\mathrm{C} 2-\mathrm{H} 2 B \cdots \mathrm{Cl} 1$ & 0.98 & 2.62 & $3.5215(1)$ & 153 \\
$\mathrm{C} 11-\mathrm{H} 11 B \cdots \mathrm{Cl} 1^{\mathrm{i}}$ & 0.98 & 2.49 & $3.4594(1)$ & 170 \\
$\mathrm{C} 209-\mathrm{H} 209 \cdots \mathrm{N}^{\mathrm{ii}}$ & 0.94 & 2.60 & $3.3722(1)$ & 140 \\
$\mathrm{C} 306-\mathrm{H} 306 \cdots \mathrm{Cl} 1 A^{\mathrm{iii}}$ & 0.94 & 2.81 & $3.7037(1)$ & 159 \\
\hline
\end{tabular}

Symmetry codes: (i) $-x+\frac{1}{2}, y+\frac{1}{2}, z$; (ii) $-x+1, y,-z-\frac{1}{2}$; (iii) $-x+\frac{1}{2},-y+\frac{3}{2}, z+\frac{1}{2}$.

Table 3

Hydrogen-bond geometry $\left(\AA,^{\circ}\right)$ for $\mathbf{1 b}$.

\begin{tabular}{lllll}
\hline$D-\mathrm{H} \cdots A$ & $D-\mathrm{H}$ & $\mathrm{H} \cdots A$ & $D \cdots A$ & $D-\mathrm{H} \cdots A$ \\
\hline $\mathrm{C} 3-\mathrm{H} 3 B \cdots \mathrm{N} 2$ & 0.98 & 2.58 & $3.4925(1)$ & 156 \\
$\mathrm{C} 102-\mathrm{H} 102 \cdots \mathrm{Cl} 1$ & 0.94 & 2.66 & $3.5216(1)$ & 153 \\
$\mathrm{C} 112-\mathrm{H} 112 \cdots \mathrm{Cl} 1^{\mathrm{i}}$ & 0.94 & 2.77 & $3.6470(1)$ & 155 \\
$\mathrm{C} 210-\mathrm{H} 210 \cdots \mathrm{N} 2^{\mathrm{ii}}$ & 0.94 & 2.61 & $3.4554(1)$ & 150 \\
$\mathrm{C} 303-\mathrm{H} 303 \cdots \mathrm{N} 1^{\mathrm{iii}}$ & 0.94 & 2.48 & $3.2064(1)$ & 134 \\
\hline
\end{tabular}

Symmetry codes: (i) $\quad-x+\frac{5}{2}, y+\frac{1}{2},-z+\frac{3}{2} ; \quad$ (ii) $\quad x+\frac{1}{2},-y+\frac{1}{2}, z+\frac{1}{2}$; $-x+2,-y+1,-z+1$.

Table 4

Hydrogen-bond geometry $\left(\AA{ }^{\circ}\right)$ for 3 .

\begin{tabular}{lllll}
\hline$D-\mathrm{H} \cdots A$ & $D-\mathrm{H}$ & $\mathrm{H} \cdots A$ & $D \cdots A$ & $D-\mathrm{H} \cdots A$ \\
\hline $\mathrm{O} 1-\mathrm{H} 1 A \cdots \mathrm{N} 4$ & 0.83 & 2.02 & $2.8181(1)$ & 162 \\
$\mathrm{O} 2-\mathrm{H} 2 A \cdots \mathrm{N} 3$ & 0.83 & 2.16 & $2.9486(1)$ & 158 \\
\hline
\end{tabular}

connected to the complex via hydrogen bonds, one to the triazenido group $(\mathrm{O} 2-\mathrm{H} 2 A \cdots \mathrm{N} 3=2.16 \AA)$ and the other to the cyanido ligand $(\mathrm{O} 1-\mathrm{H} 1 A \cdots \mathrm{N} 4=2.02 \AA)$. 
Table 5

Experimental details.

1b

Crystal data

Chemical formula

$M_{\mathrm{r}}$

Crystal system, space group

Temperature (K)

$a, b, c(\AA)$

$\alpha, \beta, \gamma\left({ }^{\circ}\right)$

$V\left(\AA^{3}\right)$

$Z$

Radiation type

$\mu\left(\mathrm{mm}^{-1}\right)$

Crystal size (mm)

Data collection

Diffractometer

No. of measured, independent and

observed $[I>2 \sigma(I)]$ reflections

$R_{\text {int }}$

$(\sin \theta / \lambda)_{\max }\left(\AA^{-1}\right)$

Refinemen

$R\left[F^{2}>2 \sigma\left(F^{2}\right)\right], w R\left(F^{2}\right), S$

No. of reflections

No. of parameters

No. of restraints

$\mathrm{H}$-atom treatment

$\Delta \rho_{\max }, \Delta \rho_{\min }\left(\mathrm{e} \AA^{-3}\right)$

\section{$\left[\operatorname{Ir}(\mathrm{CN}) \mathrm{ClH}\left(\mathrm{C}_{51} \mathrm{H}_{44} \mathrm{P}_{4}\right)\right] \cdot \mathrm{C}_{2} \mathrm{H}_{3} \mathrm{~N}$}

1076.47

Monoclinic, $P 2_{1} / n$

233

18.3264 (3), 13.6459 (2),

19.3010 (4)

90, 101.803 (1), 90

4724.74 (14)

4

Mo $K \alpha$

3.06

$0.18 \times 0.07 \times 0.02$

Nonius KappaCCD

$25915,8317,6345$

0.086

0.595

$0.040,0.069,1.03$

8317

564

0

$\mathrm{H}$ atoms treated by a mixture of

independent and constrained

refinement

$0.56,-1.04$
2

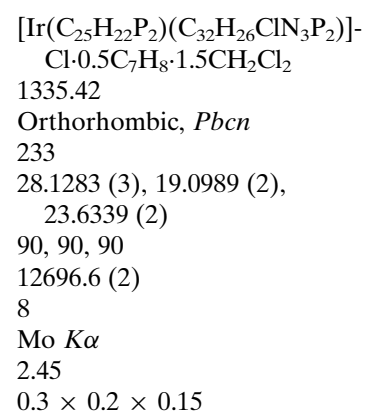

3

Nonius KappaCCD

73594, 11155, 9002

0.039

0.595

$\left[\operatorname{Ir}(\mathrm{CN})\left(\mathrm{C}_{23} \mathrm{H}_{22} \mathrm{P}_{2}\right)\left(\mathrm{C}_{34} \mathrm{H}_{26} \mathrm{Cl}-\right.\right.$ $\left.\left.\mathrm{N}_{3} \mathrm{P}_{2}\right)\right] \cdot 2 \mathrm{CH}_{4} \mathrm{O}$

1216.61

Triclinic, $P \overline{1}$

223

11.1683 (1), 12.7805 (2),

$20.0591(3)$

98.475 (1), 93.122 (1), 109.336 (1)

$2655.75(6)$

2

Mo $K \alpha$

2.73

$0.31 \times 0.30 \times 0.12$

Nonius KappaCCD

20072, 10396, 9895

0.034

0.617

$0.042,0.126,1.05$

11155

724

30

$\mathrm{H}$-atom parameters constrained

$1.66,-0.73$

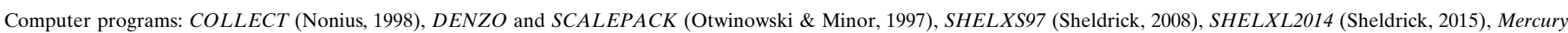
(Macrae et al., 2008) and publCIF (Westrip, 2010).

\section{Synthesis and crystallization}

The syntheses of the title compounds are summarized in the general reaction scheme for the synthesis and crystallization of $\mathbf{2}, \mathbf{1 b}$ and $\mathbf{3}$, starting from $\mathbf{1 a}(\mathbf{1 c}$ is only mentioned for comparative purposes - see Structural commentary). All preparations were carried out under an inert atmosphere $\left(\mathrm{N}_{2}\right)$ using standard Schlenk techniques. ${ }^{1} \mathrm{H},{ }^{13} \mathrm{C}$ and ${ }^{31} \mathrm{P}$ NMR spectra were recorded on a Bruker DPX 300 NMR spectrometer $(300 \mathrm{MHz})$ and were referenced against ${ }^{13} \mathrm{C} /{ }^{1} \mathrm{H}$ solvent peaks or an external $85 \% \mathrm{H}_{3} \mathrm{PO}_{4}$ standard, respectively. The phosphorus atoms in the NMR data are labelled in the same way as in the figures.

Synthesis of complex 2: 1a is formed upon stirring a mixture of $[\mathrm{IrCl}(\mathrm{cod})]_{2} \quad(8.5 \mathrm{mg}, \quad 0.0125 \mathrm{mmol}), \quad\left[\mathrm{CH}(\mathrm{dppm})_{2}\right] \mathrm{Cl}$ (20.5 mg, $0.025 \mathrm{mmol}$ ), (Reitsamer et al., 2012), $\mathrm{MeCN}$ $(0.1 \mathrm{ml})$ and $\mathrm{MeOH}(0.5 \mathrm{ml})$ for $25 \mathrm{~min}$. After this, a solution of 1-azido-4-chlorobenzene $\left(0.5 \mathrm{~mol} \mathrm{~L}^{-1}\right.$ in methyl-tert-butyl ether, $0.1 \mathrm{ml}, 0.050 \mathrm{mmol}$ ) and basic $\mathrm{Al}_{2} \mathrm{O}_{3}(30 \mathrm{mg})$ were subsequently added and the suspension was stirred for $5 \mathrm{~min}$. The liquid part was separated, and the volatiles evaporated in vacuo. The residue was then dissolved in $\mathrm{CH}_{2} \mathrm{Cl}_{2}$ /toluene 1:2. Slow evaporation gave crystalline 2.

${ }^{31} \mathrm{P}\left\{{ }^{1} \mathrm{H}\right\}$-NMR $(\mathrm{MeCN} / \mathrm{MeOH} 1: 5): \delta=16.1\left(\mathrm{P} 1, d d d ; J_{\mathrm{P} 1 \mathrm{P} 2}=\right.$ $\left.30.6, J_{\mathrm{P} 1 \mathrm{P} 3}=6.1, J_{\mathrm{P} 1 \mathrm{P} 4}=33.7 \mathrm{~Hz}\right) ; 15.6\left(\mathrm{P} 2, d d d ; J_{\mathrm{P} 2 \mathrm{P} 3}=44.4\right.$, $\left.J_{\mathrm{P} 2 \mathrm{P} 4}=13.8 \mathrm{~Hz}\right) ;-37.4\left(\mathrm{P} 3, d d d ; J_{\mathrm{P} 3 \mathrm{P} 4}=52.0 \mathrm{~Hz}\right) ;-20.9(\mathrm{P} 4$, $d d d$ ) ppm. ${ }^{13} \mathrm{C}\left\{{ }^{1} \mathrm{H}\right\}$-NMR (MeCN/MeOH 1:5): $\delta=169.5$ (C1, $\left.d d d d ; J_{\mathrm{C} 1 \mathrm{P} 1}=2.5, J_{\mathrm{C} 1 \mathrm{P} 2}=60.0, J_{\mathrm{C} 1 \mathrm{P} 3}=85.0, J_{\mathrm{C} 1 \mathrm{P} 4}=8.3 \mathrm{~Hz}\right)$ ppm.

Synthesis of coordination compounds 1b and 3: A mixture of $\quad[\mathrm{IrCl}(\mathrm{cod})]_{2} \quad(8.5 \mathrm{mg}, \quad 0.0125 \mathrm{mmol}), \quad\left[\mathrm{CH}(\mathrm{dppm})_{2}\right] \mathrm{Cl}$ $(20.5 \mathrm{mg}, 0.025 \mathrm{mmol}), \mathrm{MeCN}(0.1 \mathrm{ml})$ and $\mathrm{MeOH}(0.4 \mathrm{ml})$ was stirred for $25 \mathrm{~min}$. Then, a solution of $\mathrm{KCN}(2 \mathrm{mg}$, $0.03 \mathrm{mmol})$ in $\mathrm{MeOH}(0.2 \mathrm{ml})$ was added and the mixture stirred for $1 \mathrm{~min}$. Thereafter, a solution of 1-azido-4-chlorobenzene in MTBE $\left(0.1 \mathrm{ml}\right.$, $\mathrm{mol} \mathrm{L}^{-1}$ in MTBE, $\left.0.05 \mathrm{mmol}\right)$ was added. Yellow crystals of $\mathbf{1 b}$ formed within a few hours. Within $14 \mathrm{~d}$, the orange crystals of $\mathbf{1 b}$ disappeared, and colourless crystals of $\mathbf{3}$ had developed.

1b ${ }^{31} \mathrm{P}\left\{{ }^{1} \mathrm{H}\right\}$-NMR (MeCN/MeOH 1:6): $\delta=-0.3(\mathrm{P} 1 / \mathrm{P} 4, v t$, $\left.N_{\mathrm{P} 1 / \mathrm{P} 4 \mathrm{P} 2 / \mathrm{P} 3}=67.3 \mathrm{~Hz}\right) ; 28.2(\mathrm{P} 2 / \mathrm{P} 3, v t) \mathrm{ppm} .{ }^{13} \mathrm{C}\left\{{ }^{1} \mathrm{H}\right\}-\mathrm{NMR}$ $(\mathrm{MeCN} / \mathrm{MeOH} 1: 6): \delta=-36.5\left(\mathrm{C} 1, t, J_{\mathrm{C} 1 \mathrm{P} 2 / \mathrm{P} 3}=100.1 \mathrm{~Hz}\right) \mathrm{ppm}$. ${ }^{1} \mathrm{H}-\mathrm{NMR}(\mathrm{MeCN} / \mathrm{MeOH} 1: 6): \delta=-12.2(\mathrm{H} 1, s, 1 \mathrm{H}) \mathrm{ppm}$.

$3^{31} \mathrm{P}\left\{{ }^{1} \mathrm{H}\right\}-\mathrm{NMR}\left(\mathrm{CHCl}_{3} / \mathrm{MeOH} 1: 1\right): \delta=0.3\left(\mathrm{P} 1, d d d, J_{\mathrm{P} 1 \mathrm{P} 2}=\right.$ $\left.153.0, J_{\mathrm{P} 1 \mathrm{P} 3}=376.3, J_{\mathrm{P} 1 \mathrm{P} 4}=18.4 \mathrm{~Hz}\right) ; 55.2\left(\mathrm{P} 2, d d, J_{\mathrm{P} 2 \mathrm{P} 3}=\right.$ $49.0 \mathrm{~Hz}) ;-57.0\left(\mathrm{P} 3, d d d, J_{\mathrm{P} 3 \mathrm{P} 4}=27.5 \mathrm{~Hz}\right) ;-67.1(\mathrm{P} 4, d d) \mathrm{ppm}$. ${ }^{13} \mathrm{C}-\mathrm{NMR}\left(\mathrm{CHCl}_{3} / \mathrm{MeOH} 1: 1\right): \delta=72.2\left(\mathrm{C} 1, d d, J_{\mathrm{C} 1 \mathrm{P} 2}=38.7\right.$, $\left.J_{\mathrm{C} 1 \mathrm{H} 1}=142.1 \mathrm{~Hz}\right) \mathrm{ppm}$.

\section{Refinement}

Crystal data, data collection and structure refinement details are summarized in Table 5. Complex $\mathbf{2}$ involves a 2:1 positional disorder of the anion $\mathrm{Cl} 1 \mathrm{Cl} 1 \mathrm{~A}$. The solvent molecules $\mathrm{CH}_{2} \mathrm{Cl}_{2}$ 
and $\mathrm{C}_{7} \mathrm{H}_{8}$ show occupational disorder, with the toluene molecule exhibiting additional 1:1 positional disorder with some nearly overlying carbon atoms. We propose a correlation between $\mathrm{CH}_{2} \mathrm{Cl}_{2}$ and $\mathrm{C}_{7} \mathrm{H}_{8}$, because of short intermolecular $\mathrm{Cl}$..C contacts. Therefore, the two solvent molecules $\mathrm{Cl} 3$ / $\mathrm{C} 10-\mathrm{Cl} 4$ and $\mathrm{Cl} 5 / \mathrm{C} 11-\mathrm{Cl} 6$ have an occupancy of 0.75 and the 'two' toluene molecules, C12-C18 and C19-C25, an occupancy of 0.25 . Several bond restraints were used to refine the toluene carbon atoms reasonably isotropically. The hydride hydrogen of $\mathbf{1 b}$ was localized and refined isotropically without restraints. The hydrogen atoms at $\mathrm{C} 1$ and $\mathrm{C} 2$ of $\mathbf{3}$ were localized and refined with isotropic displacement parameters. All other $\mathrm{H}$ atoms were positioned geometrically $(\mathrm{C}-\mathrm{H}=0.94-0.98 \AA$ ) and refined as riding with $U_{\text {iso }}(\mathrm{H})=1.2-1.5 U_{\text {eq }}(\mathrm{C})$.

\section{References}

Chauvin, R. \& Canac, Y. (2010). Editors. Transition Metal Complexes of Neutral eta1-Carbon Ligands (Topics in Organometallic Chemistry, Vol. 30). Berlin, Heidelberg: Springer.

Ishida, H. (2000). Z. Naturforsch. Teil A, 55, 769-771.

Khramov, D. M. \& Bielawski, C. W. (2005). Chem. Commun. pp. 4958-4960.

Kolodiazhnyi, O. I. (1999). Phosphorus Ylides. Weinheim: Wiley$\mathrm{VCH}$.
Macrae, C. F., Bruno, I. J., Chisholm, J. A., Edgington, P. R., McCabe, P., Pidcock, E., Rodriguez-Monge, L., Taylor, R., van de Streek, J. \& Wood, P. A. (2008). J. Appl. Cryst. 41, 466-470.

Nonius (1998). COLLECT. Nonius BV, Delft, The Netherlands.

Otwinowski, Z. \& Minor, W. (1997). Methods in Enzymology, Vol. 276, Macromolecular Crystallography, Part A, edited by C. W. Carter Jr \& R. M. Sweet, pp. 307-326. New York: Academic Press.

Partl, G. J., Nussbaumer, F., Schlapp-Hackl, I., Schuh, W., Kopacka, H., Wurst, K. \& Peringer, P. (2018). Acta Cryst. E74, 846-852.

Petz, W. \& Frenking, G. (2010). Topics in Organometallic Chemistry, Vol. 30, edited by R. Chauvin and Y. Canac, pp. 49-92. Berlin, Heidelberg: Springer.

Poverenov, E. \& Milstein, D. (2013). Topics in Organometallic Chemistry, Vol. 40, edited by G. van Koten and D. Milstein, pp. 2147. Berlin, Heidelberg: Springer.

Reitsamer, C., Stallinger, S., Schuh, W., Kopacka, H., Wurst, K., Obendorf, D. \& Peringer, P. (2012). Dalton Trans. 41, 35033514.

Schlapp-Hackl, I., Pauer, B., Falschlunger, C., Schuh, W., Kopacka, H., Wurst, K. \& Peringer, P. (2018). Acta Cryst. E74, 16431647.

Sheldrick, G. M. (2008). Acta Cryst. A64, 112-122.

Sheldrick, G. M. (2015). Acta Cryst. C71, 3-8.

Takayama, T., Mitsumori, T., Kawano, M., Sekine, A., Uekusa, H., Ohashi, Y. \& Sugawara, T. (2010). Acta Cryst. B66, 639-646.

Westrip, S. P. (2010). J. Appl. Cryst. 43, 920-925. 


\section{supporting information}

Acta Cryst. (2019). E75, 75-80 [https://doi.org/10.1107/S2056989018017644]

Crystal structures of two PCN pincer iridium complexes and one PCP pincer carbodiphosphorane iridium intermediate: substitution of one phosphine moiety of a carbodiphosphorane by an organic azide

Gabriel Julian Partl, Felix Nussbaumer, Walter Schuh, Holger Kopacka, Klaus Wurst and Paul Peringer

Computing details

For all structures, data collection: COLLECT (Nonius, 1998); data reduction: DENZO and SCALEPACK (Otwinowski \& Minor, 1997); program(s) used to solve structure: SHELXS97 (Sheldrick, 2008); program(s) used to refine structure: SHELXL2014 (Sheldrick, 2015); software used to prepare material for publication: Mercury (Macrae et al., 2008) and publCIF (Westrip, 2010).

Chloridocyanidohydrido(1,1,3,3,5,5,7,7-octaphenyl-1,3$\lambda^{5}, 5 \lambda^{4}, 7$-tetraphospha- $\kappa^{2} P^{1}, P^{7}$-hept-3-en-4-yl)iridium(III) acetonitrile monosolvate $(\mathbf{1 b})$

Crystal data

$\left[\operatorname{Ir}(\mathrm{CN}) \mathrm{ClH}\left(\mathrm{C}_{51} \mathrm{H}_{44} \mathrm{P}_{4}\right)\right] \cdot \mathrm{C}_{2} \mathrm{H}_{3} \mathrm{~N}$

$M_{r}=1076.47$

Monoclinic, $P 2_{1} / n$

$a=18.3264$ (3) $\AA$

$b=13.6459(2) \AA$

$c=19.3010$ (4) $\AA$

$\beta=101.803(1)^{\circ}$

$V=4724.74(14) \AA^{3}$

$Z=4$

$F(000)=2160$

$D_{\mathrm{x}}=1.513 \mathrm{Mg} \mathrm{m}^{-3}$

Mo $K \alpha$ radiation, $\lambda=0.71073 \AA$

Cell parameters from 52211 reflections

$\theta=1.0-25.0^{\circ}$

$\mu=3.06 \mathrm{~mm}^{-1}$

$T=233 \mathrm{~K}$

Prism, yellow

$0.18 \times 0.07 \times 0.02 \mathrm{~mm}$

Data collection

Nonius KappaCCD

diffractometer

phi- and $\omega$-scans

25915 measured reflections

8317 independent reflections

6345 reflections with $I>2 \sigma(I)$

$R_{\text {int }}=0.086$

$\theta_{\text {max }}=25.0^{\circ}, \theta_{\min }=1.4^{\circ}$

$h=-21 \rightarrow 20$

$k=-16 \rightarrow 16$

$l=-22 \rightarrow 22$

Refinement

Refinement on $F^{2}$

Least-squares matrix: full

$R\left[F^{2}>2 \sigma\left(F^{2}\right)\right]=0.040$

$w R\left(F^{2}\right)=0.069$

$S=1.03$

8317 reflections
564 parameters

0 restraints

Hydrogen site location: mixed

$\mathrm{H}$ atoms treated by a mixture of independent and constrained refinement 
$w=1 /\left[\sigma^{2}\left(F_{\mathrm{o}}^{2}\right)+(0.021 P)^{2}+3.0711 P\right]$

where $P=\left(F_{\mathrm{o}}^{2}+2 F_{\mathrm{c}}^{2}\right) / 3$

$(\Delta / \sigma)_{\max }=0.003$

$$
\Delta \rho_{\max }=0.56 \text { e } \AA^{-3}
$$

$\Delta \rho_{\min }=-1.04$ e $\AA^{-3}$

\section{Special details}

Experimental. All data sets were measured with several scans to increase the number of redundant reflections. In our experience this method of averaging redundant reflections replaces in a good approximation semi-empirical absorptions methods (absorption correction programs like SORTAV lead to no better data sets).

Geometry. All esds (except the esd in the dihedral angle between two 1.s. planes) are estimated using the full covariance matrix. The cell esds are taken into account individually in the estimation of esds in distances, angles and torsion angles; correlations between esds in cell parameters are only used when they are defined by crystal symmetry. An approximate (isotropic) treatment of cell esds is used for estimating esds involving l.s. planes.

Refinement. Small crystal with low diffraction at higher 2 theta angles. Hydrogen at Ir were localized and refined isotropically without restraints.

Fractional atomic coordinates and isotropic or equivalent isotropic displacement parameters $\left(\hat{A}^{2}\right)$

\begin{tabular}{|c|c|c|c|c|}
\hline & $x$ & $y$ & $z$ & $U_{\text {iso }} * / U_{\text {eq }}$ \\
\hline Ir1 & $1.08853(2)$ & $0.20725(2)$ & $0.71435(2)$ & $0.02191(7)$ \\
\hline H1 & 1.1054 (19) & $0.172(3)$ & $0.790(2)$ & $0.020(11)^{*}$ \\
\hline $\mathrm{P} 1$ & $1.20178(6)$ & $0.28650(9)$ & $0.73888(6)$ & 0.0239 \\
\hline $\mathrm{P} 2$ & $1.10074(7)$ & $0.40362(9)$ & $0.80500(6)$ & 0.0269 (3) \\
\hline P3 & $0.94640(6)$ & $0.35370(9)$ & $0.72144(6)$ & $0.0251(3)$ \\
\hline P4 & $0.97290(6)$ & $0.13964(9)$ & $0.70074(6)$ & 0.0260 \\
\hline $\mathrm{Cl1}$ & $1.14187(7)$ & $0.05475(9)$ & $0.68044(7)$ & 0.0383 \\
\hline $\mathrm{C} 4$ & 1.0599 (2) & $0.2604(3)$ & $0.6113(3)$ & $0.0278(11)$ \\
\hline N1 & $1.0392(2)$ & $0.2906(3)$ & $0.5559(2)$ & $0.0486(12)$ \\
\hline $\mathrm{C} 1$ & $1.0385(2)$ & $0.3332(3)$ & $0.7501(2)$ & $0.0250(11)$ \\
\hline $\mathrm{C} 2$ & $1.1831(2)$ & $0.4124(3)$ & $0.7662(3)$ & 0.0279 \\
\hline $\mathrm{H} 2 \mathrm{~A}$ & 1.2256 & 0.4375 & 0.8009 & $0.034 *$ \\
\hline $\mathrm{H} 2 \mathrm{~B}$ & 1.1738 & 0.4565 & 0.7252 & $0.034 *$ \\
\hline $\mathrm{C} 3$ & 0.9069 (2) & $0.2410(3)$ & $0.6795(3)$ & $0.0314(12)$ \\
\hline $\mathrm{H} 3 \mathrm{~A}$ & 0.8941 & 0.2502 & 0.6281 & $0.038 *$ \\
\hline Н3B & 0.8610 & 0.2252 & 0.6958 & $0.038 *$ \\
\hline C101 & $1.2526(2)$ & $0.3058(3)$ & $0.6676(2)$ & $0.0268(11)$ \\
\hline C102 & 1.2477 & $0.2350(4)$ & $0.6151(3)$ & $0.0390(13)$ \\
\hline H102 & 1.2189 & 0.1783 & 0.6167 & $0.047^{*}$ \\
\hline C103 & $1.2852(3)$ & $0.2482(4)$ & 0.5605 & $0.0518(16)$ \\
\hline H103 & 1.2825 & 0.1996 & 0.5256 & $0.062 *$ \\
\hline C104 & 1.3261 & $0.3312(4)$ & 0.5567 & $0.0473(15)$ \\
\hline H104 & 1.3509 & 0.3397 & 0.5191 & $0.057^{*}$ \\
\hline $\mathrm{C} 105$ & 1.3309 (3) & $0.4013(4)$ & $0.6076(3)$ & $0.0468(15)$ \\
\hline H105 & 1.3589 & 0.4583 & 0.6046 & $0.056^{*}$ \\
\hline C106 & $1.2951(3)$ & $0.3896(4)$ & $0.6638(3)$ & $0.0386(13)$ \\
\hline H106 & 1.2994 & 0.4380 & 0.6991 & $0.046^{*}$ \\
\hline $\mathrm{C} 107$ & $1.2722(2)$ & 0.2390 & $0.8118(2)$ & 0.0260 \\
\hline C108 & $1.2622(3)$ & $0.1486(4)$ & 0.8407 (3) & $0.0377(13)$ \\
\hline H108 & 1.2199 & 0.1111 & 0.8210 & $0.045^{*}$ \\
\hline C109 & $1.3127(3)$ & $0.1120(4)$ & 0.8979 & $0.0500(15)$ \\
\hline
\end{tabular}




\begin{tabular}{|c|c|c|c|c|}
\hline H109 & 1.3051 & 0.0499 & 0.9164 & $0.060^{*}$ \\
\hline $\mathrm{C} 110$ & $1.3737(3)$ & $0.1661(4)$ & $0.9276(3)$ & $0.0468(15)$ \\
\hline H110 & 1.4071 & 0.1427 & 0.9678 & $0.056^{*}$ \\
\hline C111 & $1.3864(3)$ & $0.2544(4)$ & $0.8988(3)$ & $0.0451(14)$ \\
\hline H111 & 1.4295 & 0.2902 & 0.9183 & $0.054 *$ \\
\hline $\mathrm{C} 112$ & $1.3361(2)$ & $0.2916(4)$ & $0.8411(3)$ & $0.0380(12)$ \\
\hline H112 & 1.3452 & 0.3525 & 0.8217 & $0.046^{*}$ \\
\hline C201 & $1.0711(3)$ & $0.5264(4)$ & $0.8215(3)$ & $0.0333(12)$ \\
\hline C202 & $1.0762(3)$ & $0.6032(4)$ & $0.7762(3)$ & $0.0463(14)$ \\
\hline $\mathrm{H} 202$ & 1.0988 & 0.5933 & 0.7372 & $0.056^{*}$ \\
\hline C203 & $1.0482(3)$ & $0.6950(4)$ & $0.7877(4)$ & $0.0652(18)$ \\
\hline $\mathrm{H} 203$ & 1.0515 & 0.7468 & 0.7564 & $0.078^{*}$ \\
\hline $\mathrm{C} 204$ & $1.0159(3)$ & $0.7098(5)$ & $0.8444(4)$ & 0.0659 (19) \\
\hline H204 & 0.9963 & 0.7718 & 0.8515 & $0.079^{*}$ \\
\hline C205 & $1.0116(4)$ & $0.6357(5)$ & $0.8910(4)$ & $0.071(2)$ \\
\hline $\mathrm{H} 205$ & 0.9900 & 0.6469 & 0.9305 & $0.085^{*}$ \\
\hline C206 & $1.0394(3)$ & $0.5437(4)$ & $0.8798(3)$ & $0.0479(15)$ \\
\hline H206 & 1.0367 & 0.4927 & 0.9119 & $0.058^{*}$ \\
\hline C207 & $1.1346(3)$ & $0.3515(4)$ & $0.8923(3)$ & $0.0338(12)$ \\
\hline C208 & $1.0916(3)$ & $0.2794(4)$ & $0.9159(3)$ & $0.0468(14)$ \\
\hline H208 & 1.0472 & 0.2581 & 0.8864 & $0.056^{*}$ \\
\hline C209 & $1.1141(4)$ & $0.2391(5)$ & 0.9828 (3) & 0.0690 (19) \\
\hline H209 & 1.0840 & 0.1918 & 0.9987 & $0.083^{*}$ \\
\hline $\mathrm{C} 210$ & $1.1788(4)$ & $0.2670(5)$ & $1.0253(3)$ & $0.073(2)$ \\
\hline $\mathrm{H} 210$ & 1.1942 & 0.2378 & 1.0700 & $0.088^{*}$ \\
\hline C211 & 1.2217 (4) & $0.3377(6)$ & $1.0034(3)$ & 0.0672 (19) \\
\hline $\mathrm{H} 211$ & 1.2661 & 0.3577 & 1.0336 & $0.081^{*}$ \\
\hline $\mathrm{C} 212$ & $1.2001(3)$ & $0.3807(4)$ & $0.9364(3)$ & $0.0499(15)$ \\
\hline $\mathrm{H} 212$ & 1.2300 & 0.4291 & 0.9215 & $0.060^{*}$ \\
\hline C301 & $0.9145(2)$ & $0.4436(3)$ & $0.6526(2)$ & $0.0277(11)$ \\
\hline C302 & $0.9642(3)$ & $0.4980(4)$ & $0.6233(3)$ & 0.0395 (13) \\
\hline H302 & 1.0157 & 0.4913 & 0.6408 & $0.047^{*}$ \\
\hline C303 & $0.9379(3)$ & $0.5629(4)$ & $0.5678(3)$ & $0.0450(14)$ \\
\hline H303 & 0.9720 & 0.6004 & 0.5486 & $0.054^{*}$ \\
\hline C304 & $0.8632(3)$ & $0.5723(4)$ & $0.5411(3)$ & $0.0451(14)$ \\
\hline H304 & 0.8458 & 0.6172 & 0.5045 & $0.054^{*}$ \\
\hline C305 & $0.8135(3)$ & $0.5153(4)$ & $0.5686(3)$ & $0.0518(16)$ \\
\hline H305 & 0.7621 & 0.5205 & 0.5498 & $0.062 *$ \\
\hline C306 & $0.8386(3)$ & $0.4512(4)$ & $0.6230(3)$ & $0.0406(14)$ \\
\hline H306 & 0.8044 & 0.4119 & 0.6406 & $0.049^{*}$ \\
\hline C307 & $0.8966(2)$ & $0.3862(4)$ & $0.7904(3)$ & $0.0309(12)$ \\
\hline C308 & $0.8912(3)$ & 0.3181 (4) & 0.8417 (3) & $0.0546(17)$ \\
\hline H308 & 0.9099 & 0.2545 & 0.8382 & $0.066^{*}$ \\
\hline C309 & $0.8585(4)$ & $0.3418(5)$ & $0.8983(4)$ & $0.081(2)$ \\
\hline H309 & 0.8551 & 0.2943 & 0.9328 & $0.097 *$ \\
\hline $\mathrm{C} 310$ & $0.8316(4)$ & $0.4334(5)$ & $0.9041(4)$ & $0.078(2)$ \\
\hline H310 & 0.8102 & 0.4495 & 0.9429 & $0.094^{*}$ \\
\hline C311 & 0.8354 (3) & $0.5022(5)$ & $0.8537(3)$ & $0.0642(18)$ \\
\hline
\end{tabular}




\begin{tabular}{|c|c|c|c|c|}
\hline H311 & 0.8160 & 0.5653 & 0.8574 & $0.077^{*}$ \\
\hline C312 & $0.8682(3)$ & $0.4788(4)$ & 0.7965 & $0.0463(14)$ \\
\hline H312 & 0.8710 & 0.5264 & 0.7620 & $0.056^{*}$ \\
\hline C401 & 0.9435 & $0.0522(4)$ & $0.6292(3)$ & $0.0346(13)$ \\
\hline C402 & $0.9211(3)$ & 0.0818 & $0.5597(3)$ & $0.0501(15)$ \\
\hline H402 & 0.9182 & 0.1492 & 0.5494 & $0.060 *$ \\
\hline C403 & 0.9029 & $0.0156(5)$ & $0.5046(4)$ & 0.0666 (19) \\
\hline H403 & 0.8876 & 0.0381 & 0.4579 & $0.080 *$ \\
\hline C404 & $0.9073(3)$ & $-0.0821(5)$ & $0.5184(4)$ & $0.0650(19)$ \\
\hline H404 & 0.8935 & -0.1273 & 0.4813 & $0.078^{*}$ \\
\hline C405 & $0.9314(4)$ & $-0.1145(5)$ & $0.5855(4)$ & $0.068(2)$ \\
\hline H405 & 0.9358 & -0.1821 & 0.5945 & $0.082 *$ \\
\hline C406 & $0.9502(3)$ & $-0.0471(4)$ & $0.6425(3)$ & $0.0548(16)$ \\
\hline H406 & 0.9671 & -0.0700 & 0.6889 & $0.066^{*}$ \\
\hline C407 & $0.9433(3)$ & 0.0784 & 0.7744 & $0.0332(12)$ \\
\hline C408 & $0.9928(3)$ & $0.0573(4)$ & 0.8363 & $0.0524(16)$ \\
\hline H408 & 1.0433 & 0.0738 & 0.8407 & $0.063^{*}$ \\
\hline C409 & $0.9695(4)$ & $0.0124(5)$ & $0.8918(3)$ & $0.077(2)$ \\
\hline H409 & 1.0043 & -0.0032 & 0.9333 & $0.092 *$ \\
\hline C410 & $0.8955(4)$ & $-0.0098(5)$ & $0.8868(4)$ & $0.083(2)$ \\
\hline H410 & 0.8793 & -0.0385 & 0.9253 & $0.099 *$ \\
\hline C411 & $0.8463(4)$ & 0.0098 & $0.8266(5)$ & $0.084(2)$ \\
\hline H411 & 0.7958 & -0.0060 & 0.8232 & $0.101 *$ \\
\hline $\mathrm{C} 412$ & 0.8689 & $0.0531(4)$ & $0.7694(3)$ & $0.0580(17)$ \\
\hline H412 & 0.8340 & 0.0652 & 0.7273 & $0.070 *$ \\
\hline N2 & 0.7247 & $0.2498(6)$ & $0.7077(4)$ & $0.103(2)$ \\
\hline $\mathrm{C} 5$ & $0.6995(4)$ & $0.2484(5)$ & $0.7548(5)$ & $0.077(2)$ \\
\hline C6 & $0.6679(6)$ & $0.2427(8)$ & $0.8163(5)$ & $0.158(5)$ \\
\hline H6A & 0.6629 & 0.1745 & 0.8287 & $0.237^{*}$ \\
\hline H6B & 0.6191 & 0.2735 & 0.8068 & $0.237^{*}$ \\
\hline $\mathrm{H} 6 \mathrm{C}$ & 0.7000 & 0.2761 & 0.8553 & $0.237^{*}$ \\
\hline
\end{tabular}

Atomic displacement parameters $\left(\AA^{2}\right)$

\begin{tabular}{lllllll}
\hline & $U^{11}$ & $U^{22}$ & $U^{33}$ & $U^{12}$ & $U^{13}$ & $U^{23}$ \\
\hline Ir1 & $0.02357(11)$ & $0.02054(11)$ & $0.02067(10)$ & $0.00045(9)$ & $0.00230(7)$ & $-0.00009(9)$ \\
P1 & $0.0248(6)$ & $0.0235(7)$ & $0.0224(6)$ & $0.0009(5)$ & $0.0023(5)$ & $-0.0005(6)$ \\
P2 & $0.0297(7)$ & $0.0253(7)$ & $0.0254(7)$ & $0.0017(5)$ & $0.0049(5)$ & $-0.0042(6)$ \\
P3 & $0.0262(7)$ & $0.0263(7)$ & $0.0235(7)$ & $0.0017(5)$ & $0.0062(5)$ & $0.0006(6)$ \\
P4 & $0.0261(7)$ & $0.0241(7)$ & $0.0268(7)$ & $-0.0007(5)$ & $0.0028(5)$ & $0.0003(6)$ \\
C11 & $0.0443(8)$ & $0.0313(7)$ & $0.0376(8)$ & $0.0043(6)$ & $0.0040(6)$ & $-0.0016(6)$ \\
C4 & $0.027(3)$ & $0.023(3)$ & $0.034(3)$ & $-0.001(2)$ & $0.008(2)$ & $-0.002(2)$ \\
N1 & $0.063(3)$ & $0.050(3)$ & $0.031(3)$ & $0.001(2)$ & $0.004(2)$ & $0.013(3)$ \\
C1 & $0.024(3)$ & $0.030(3)$ & $0.022(3)$ & $-0.002(2)$ & $0.007(2)$ & $0.002(2)$ \\
C2 & $0.027(3)$ & $0.023(3)$ & $0.034(3)$ & $0.000(2)$ & $0.006(2)$ & $0.000(2)$ \\
C3 & $0.032(3)$ & $0.030(3)$ & $0.032(3)$ & $-0.002(2)$ & $0.004(2)$ & $0.001(2)$ \\
C101 & $0.025(3)$ & $0.029(3)$ & $0.027(3)$ & $0.004(2)$ & $0.007(2)$ & $0.008(2)$ \\
C102 & $0.043(3)$ & $0.035(3)$ & $0.041(3)$ & $-0.008(2)$ & $0.014(3)$ & $-0.013(3)$
\end{tabular}




\begin{tabular}{|c|c|c|c|c|c|c|}
\hline $\mathrm{C} 103$ & $0.068(4)$ & $0.056(4)$ & $0.039(3)$ & $-0.008(3)$ & 0.028 & $-0.018(3)$ \\
\hline $\mathrm{C} 104$ & $0.046(4)$ & $0.061(4)$ & $0.041(4)$ & $0.002(3)$ & $0.023(3)$ & $0.005(3)$ \\
\hline $\mathrm{C} 105$ & $0.048(4)$ & $0.044(4)$ & $0.056(4)$ & $-0.007(3)$ & $0.027(3)$ & $0.007(3)$ \\
\hline C106 & $0.039(3)$ & $0.032(3)$ & $0.046(3)$ & $-0.002(2)$ & $0.011(3)$ & $-0.005(3)$ \\
\hline $\mathrm{C} 107$ & $0.029(3)$ & $0.022(3)$ & $0.026(3)$ & $0.004(2)$ & $0.003(2)$ & $-0.001(2)$ \\
\hline C108 & $0.031(3)$ & $0.038(3)$ & $0.039(3)$ & $-0.004(2)$ & $-0.005(2)$ & $0.006(3)$ \\
\hline C109 & 0.049 (4) & 0.048 (4) & $0.045(4)$ & $-0.002(3)$ & -0.010 & $0.017(3)$ \\
\hline $\mathrm{C} 110$ & $0.034(3)$ & $0.063(4)$ & $0.037(3)$ & $0.008(3)$ & -0.008 & $0.005(3)$ \\
\hline C111 & $0.035(3)$ & $0.050(4)$ & $0.044(3)$ & $-0.001(3)$ & -0.006 & $-0.007(3)$ \\
\hline $\mathrm{C} 112$ & $0.029(3)$ & $0.033(3)$ & $0.049(3)$ & $-0.004(2)$ & $0.001(2)$ & $-0.003(3)$ \\
\hline $\mathrm{C} 201$ & $0.031(3)$ & $0.030(3)$ & 0.038 & $0.001(2)$ & $0.005(2)$ & $-0.007(3)$ \\
\hline $\mathrm{C} 202$ & $0.045(3)$ & 0.037 (4) & $0.057(4)$ & $0.005(3)$ & $0.012(3)$ & -0.007 (3) \\
\hline $\mathrm{C} 203$ & $0.073(4)$ & $0.035(4)$ & $0.087(5)$ & $0.011(3)$ & $0.017(4)$ & $0.000(4)$ \\
\hline C204 & $0.056(4)$ & $0.040(4)$ & $0.101(6)$ & $0.011(3)$ & $0.012(4)$ & $-0.028(4)$ \\
\hline $\mathrm{C} 205$ & $0.077(5)$ & $0.060(5)$ & $0.083(5)$ & $0.012(4)$ & $0.033(4)$ & $-0.033(4)$ \\
\hline $\mathrm{C} 206$ & 0.055 (4) & 0.039 (3) & $0.052(4)$ & 0.005 & 0.018 (3) & -0.013 \\
\hline C207 & $0.035(3)$ & $0.037(3)$ & $0.028(3)$ & $0.007(2)$ & $0.001(2)$ & -0.007 (3) \\
\hline C208 & $0.058(4)$ & $0.048(4)$ & $0.032(3)$ & 0.003 & $0.005(3)$ & -0.002 \\
\hline C209 & $0.101(6)$ & $0.068(5)$ & 0.035 (4) & $0.012(4)$ & 0.009 (4) & $0.019(3)$ \\
\hline $\mathrm{C} 210$ & $0.099(6)$ & $0.088(6)$ & $0.029(4)$ & $0.032(5)$ & $0.003(4)$ & $0.008(4)$ \\
\hline C211 & $0.064(4)$ & $0.097(6)$ & $0.031(4)$ & 0.015 & -0.012 & -0.009 (4) \\
\hline $\mathrm{C} 212$ & 0.049 (4) & 0.068 (4) & $0.031(3)$ & 0.000 & $0.004(3)$ & -0.008 \\
\hline $\mathrm{C} 301$ & $0.027(3)$ & $0.027(3)$ & $0.028(3)$ & $0.003(2)$ & $0.005(2)$ & $0.002(2)$ \\
\hline $\mathrm{C} 302$ & $0.032(3)$ & 0.043 & $0.043(3)$ & $-0.002(2)$ & $0.005(2)$ & $0.012(3)$ \\
\hline C303 & 0.053 & $0.042(3)$ & $0.039(3)$ & $-0.006(3)$ & 0.009 (3) & 0.019 \\
\hline C304 & 0.055 & $0.042(3)$ & $0.036(3)$ & $0.009(3)$ & 0.005 & $0.017(3)$ \\
\hline $\mathrm{C} 305$ & $0.038(3)$ & $0.066(4)$ & $0.049(4)$ & $0.014(3)$ & $0.003(3)$ & $0.017(3)$ \\
\hline C306 & $0.035(3)$ & $0.045(3)$ & $0.043(3)$ & $0.008(2)$ & $0.008(3)$ & $0.015(3)$ \\
\hline C307 & 0.028 & $0.034(3)$ & $0.031(3)$ & $0.005(2)$ & $0.005(2)$ & -0.001 \\
\hline C308 & 0.070 & 0.050 & 0.055 & 0.018 & $0.037(3)$ & 0.011 \\
\hline C309 & $0.136(7)$ & $0.060(5)$ & $0.065(5)$ & $0.022(4)$ & $0.064(5)$ & $0.022(4)$ \\
\hline $\mathrm{C} 310$ & $0.118(6)$ & $0.076(5)$ & $0.056(5)$ & $0.015(5)$ & $0.057(4)$ & $0.004(4)$ \\
\hline C311 & 0.081 & $0.058(4)$ & $0.062(4)$ & $0.022(3)$ & $0.034(4)$ & -0.011 \\
\hline $\mathrm{C} 312$ & $0.056(4)$ & $0.045(4)$ & $0.040(3)$ & $0.013(3)$ & $0.016(3)$ & $0.007(3)$ \\
\hline $\mathrm{C} 401$ & $0.025(3)$ & $0.034(3)$ & $0.045(3)$ & $-0.007(2)$ & $0.008(2)$ & $-0.012(3)$ \\
\hline $\mathrm{C} 402$ & $0.056(4)$ & $0.055(4)$ & $0.036(3)$ & -0.013 & $0.002(3)$ & $-0.017(3)$ \\
\hline C403 & 0.059 & $0.085(6)$ & $0.054(4)$ & $-0.014(4)$ & 0.009 (3) & -0.032 \\
\hline $\mathrm{C} 404$ & $0.064(4)$ & $0.063(5)$ & $0.065(5)$ & $-0.012(4)$ & $0.007(4)$ & -0.035 \\
\hline $\mathrm{C} 405$ & $0.070(4)$ & $0.033(4)$ & $0.104(6)$ & -0.009 & $0.023(4)$ & $-0.026(4)$ \\
\hline $\mathrm{C} 406$ & $0.057(4)$ & $0.039(4)$ & $0.067(4)$ & $-0.001(3)$ & $0.009(3)$ & -0.002 \\
\hline C407 & 0.035 & 0.029 (3) & $0.037(3)$ & $-0.002(2)$ & $0.009(2)$ & $0.007(3)$ \\
\hline $\mathrm{C} 408$ & $0.044(4)$ & $0.063(4)$ & $0.052(4)$ & $0.001(3)$ & $0.015(3)$ & $0.024(3)$ \\
\hline $\mathrm{C} 409$ & $0.069(5)$ & $0.109(6)$ & $0.055(4)$ & $0.004(4)$ & $0.019(3)$ & $0.047(4)$ \\
\hline $\mathrm{C} 410$ & $0.077(5)$ & $0.094(6)$ & $0.090(6)$ & $0.002(4)$ & $0.048(5)$ & $0.049(5)$ \\
\hline C411 & $0.057(5)$ & $0.092(6)$ & $0.117(7)$ & $-0.007(4)$ & $0.048(5)$ & $0.042(5)$ \\
\hline $\mathrm{C} 412$ & $0.039(4)$ & $0.063(4)$ & $0.073(5)$ & $-0.007(3)$ & $0.013(3)$ & $0.024(4)$ \\
\hline $\mathrm{N} 2$ & $0.065(4)$ & $0.171(7)$ & $0.072(5)$ & $-0.012(4)$ & $0.011(4)$ & $-0.009(5)$ \\
\hline $\mathrm{C} 5$ & $0.088(6)$ & $0.066(5)$ & $0.082(6)$ & 0.003 & $0.029(5)$ & -0.008 \\
\hline
\end{tabular}




$\begin{array}{lllllll}\text { C6 } & 0.197(11) & 0.182(11) & 0.126(9) & -0.015(9) & 0.104(9) & -0.010(8)\end{array}$

Geometric parameters $(\AA, \stackrel{o}{)}$

\begin{tabular}{|c|c|c|c|}
\hline $\mathrm{Ir} 1-\mathrm{P} 1$ & $2.3020(12)$ & $\mathrm{C} 207-\mathrm{C} 208$ & $1.395(7)$ \\
\hline Ir1—P4 & $2.2766(12)$ & C208-C209 & $1.385(7)$ \\
\hline $\mathrm{Ir} 1-\mathrm{C} 1$ & $2.128(4)$ & $\mathrm{C} 208-\mathrm{H} 208$ & 0.9400 \\
\hline $\mathrm{Ir} 1-\mathrm{Cl1}$ & $2.4446(12)$ & $\mathrm{C} 209-\mathrm{C} 210$ & $1.352(9)$ \\
\hline $\mathrm{Ir} 1-\mathrm{C} 4$ & $2.081(5)$ & С209- & 0.9400 \\
\hline $\mathrm{Ir} 1-\mathrm{H} 1$ & $1.51(4)$ & $\mathrm{C} 210-\mathrm{C} 211$ & $1.365(9)$ \\
\hline $\mathrm{P} 1-\mathrm{C} 107$ & $1.823(5)$ & $\mathrm{C} 210-\mathrm{H} 210$ & 0.9400 \\
\hline $\mathrm{P} 1-\mathrm{C} 101$ & $1.831(4)$ & $\mathrm{C} 211-\mathrm{C} 212$ & $1.402(8)$ \\
\hline $\mathrm{P} 1-\mathrm{C} 2$ & $1.850(4)$ & $\mathrm{C} 211-\mathrm{H} 211$ & 0.9400 \\
\hline $\mathrm{P} 2-\mathrm{C} 1$ & $1.690(5)$ & $\mathrm{C} 212-\mathrm{H} 212$ & 0.9400 \\
\hline $\mathrm{P} 2-\mathrm{C} 201$ & $1.810(5)$ & $\mathrm{C} 301-\mathrm{C} 302$ & $1.382(6)$ \\
\hline $\mathrm{P} 2-\mathrm{C} 207$ & $1.817(5)$ & $\mathrm{C} 301-\mathrm{C} 306$ & $1.394(6)$ \\
\hline $\mathrm{P} 2-\mathrm{C} 2$ & $1.821(4)$ & $\mathrm{C} 302-\mathrm{C} 303$ & $1.398(7)$ \\
\hline $\mathrm{P} 3-\mathrm{C} 1$ & $1.688(4)$ & C302-H302 & 0.9400 \\
\hline $\mathrm{P} 3-\mathrm{C} 301$ & $1.816(5)$ & $\mathrm{C} 303-\mathrm{C} 304$ & $1.366(7)$ \\
\hline P3-C307 & $1.817(5)$ & C $303-\mathrm{H} 303$ & 0.9400 \\
\hline $\mathrm{P} 3-\mathrm{C} 3$ & $1.818(5)$ & $\mathrm{C} 304-\mathrm{C} 305$ & $1.385(7)$ \\
\hline $\mathrm{P} 4-\mathrm{C} 401$ & $1.822(5)$ & C304-H304 & 0.9400 \\
\hline $\mathrm{P} 4-\mathrm{C} 407$ & $1.826(5)$ & $\mathrm{C} 305-\mathrm{C} 306$ & $1.372(7)$ \\
\hline $\mathrm{P} 4-\mathrm{C} 3$ & $1.828(5)$ & $\mathrm{C} 305-\mathrm{H} 305$ & 0.9400 \\
\hline $\mathrm{C} 4-\mathrm{N} 1$ & $1.136(6)$ & C306-H306 & 0.9400 \\
\hline $\mathrm{C} 2-\mathrm{H} 2 \mathrm{~A}$ & 0.9800 & C307-C308 & $1.375(7)$ \\
\hline $\mathrm{C} 2-\mathrm{H} 2 \mathrm{~B}$ & 0.9800 & $\mathrm{C} 307-\mathrm{C} 312$ & $1.380(7)$ \\
\hline $\mathrm{C} 3-\mathrm{H} 3 \mathrm{~A}$ & 0.9800 & C308-C309 & $1.388(7)$ \\
\hline $\mathrm{C} 3-\mathrm{H} 3 \mathrm{~B}$ & 0.9800 & С $308-\mathrm{H} 308$ & 0.9400 \\
\hline C101-C102 & $1.391(6)$ & C309-C310 & $1.357(9)$ \\
\hline $\mathrm{C} 101-\mathrm{C} 106$ & $1.393(6)$ & С $309-\mathrm{H} 309$ & 0.9400 \\
\hline $\mathrm{C} 102-\mathrm{C} 103$ & $1.382(7)$ & $\mathrm{C} 310-\mathrm{C} 311$ & $1.365(8)$ \\
\hline $\mathrm{C} 102-\mathrm{H} 102$ & 0.9400 & $\mathrm{C} 310-\mathrm{H} 310$ & 0.9400 \\
\hline $\mathrm{C} 103-\mathrm{C} 104$ & $1.368(7)$ & C311-C312 & $1.397(7)$ \\
\hline $\mathrm{C} 103-\mathrm{H} 103$ & 0.9400 & С $311-\mathrm{H} 311$ & 0.9400 \\
\hline $\mathrm{C} 104-\mathrm{C} 105$ & $1.360(7)$ & C312-H312 & 0.9400 \\
\hline C104-H104 & 0.9400 & $\mathrm{C} 401-\mathrm{C} 406$ & $1.379(7)$ \\
\hline $\mathrm{C} 105-\mathrm{C} 106$ & $1.389(7)$ & $\mathrm{C} 401-\mathrm{C} 402$ & $1.381(7)$ \\
\hline $\mathrm{C} 105-\mathrm{H} 105$ & 0.9400 & $\mathrm{C} 402-\mathrm{C} 403$ & $1.383(7)$ \\
\hline C106-H106 & 0.9400 & $\mathrm{C} 402-\mathrm{H} 402$ & 0.9400 \\
\hline $\mathrm{C} 107-\mathrm{C} 108$ & $1.382(6)$ & $\mathrm{C} 403-\mathrm{C} 404$ & $1.358(9)$ \\
\hline $\mathrm{C} 107-\mathrm{C} 112$ & $1.391(6)$ & $\mathrm{C} 403-\mathrm{H} 403$ & 0.9400 \\
\hline $\mathrm{C} 108-\mathrm{C} 109$ & $1.381(7)$ & $\mathrm{C} 404-\mathrm{C} 405$ & $1.355(9)$ \\
\hline C108- $\mathrm{H} 108$ & 0.9400 & $\mathrm{C} 404-\mathrm{H} 404$ & 0.9400 \\
\hline $\mathrm{C} 109-\mathrm{C} 110$ & $1.365(7)$ & $\mathrm{C} 405-\mathrm{C} 406$ & $1.420(8)$ \\
\hline C109- & 0.9400 & $\mathrm{C} 405-\mathrm{H} 405$ & 0.9400 \\
\hline $\mathrm{C} 110-\mathrm{C} 111$ & $1.366(7)$ & $\mathrm{C} 406-\mathrm{H} 406$ & 0.9400 \\
\hline $\mathrm{C} 110-\mathrm{H} 110$ & 0.9400 & $\mathrm{C} 407-\mathrm{C} 408$ & $1.374(7)$ \\
\hline
\end{tabular}




\begin{tabular}{|c|c|c|c|}
\hline $\mathrm{C} 111-\mathrm{C} 112$ & $1.390(7)$ & $\mathrm{C} 407-\mathrm{C} 412$ & $1.389(7)$ \\
\hline C111-H111 & 0.9400 & $\mathrm{C} 408-\mathrm{C} 409$ & $1.376(7)$ \\
\hline $\mathrm{C} 112-\mathrm{H} 112$ & 0.9400 & $\mathrm{C} 408-\mathrm{H} 408$ & 0.9400 \\
\hline $\mathrm{C} 201-\mathrm{C} 202$ & $1.381(7)$ & $\mathrm{C} 409-\mathrm{C} 410$ & $1.374(8)$ \\
\hline $\mathrm{C} 201-\mathrm{C} 206$ & $1.388(7)$ & C409-H409 & 0.9400 \\
\hline $\mathrm{C} 202-\mathrm{C} 203$ & $1.388(7)$ & $\mathrm{C} 410-\mathrm{C} 411$ & $1.344(9)$ \\
\hline C202-H202 & 0.9400 & $\mathrm{C} 410-\mathrm{H} 410$ & 0.9400 \\
\hline $\mathrm{C} 203-\mathrm{C} 204$ & $1.361(9)$ & C411-C412 & $1.388(8)$ \\
\hline С203-H 203 & 0.9400 & C411-H411 & 0.9400 \\
\hline $\mathrm{C} 204-\mathrm{C} 205$ & $1.367(9)$ & $\mathrm{C} 412-\mathrm{H} 412$ & 0.9400 \\
\hline C204-H204 & 0.9400 & $\mathrm{~N} 2-\mathrm{C} 5$ & $1.101(8)$ \\
\hline $\mathrm{C} 205-\mathrm{C} 206$ & $1.388(8)$ & $\mathrm{C} 5-\mathrm{C} 6$ & $1.427(10)$ \\
\hline $\mathrm{C} 205-\mathrm{H} 205$ & 0.9400 & C6-H6A & 0.9700 \\
\hline $\mathrm{C} 206-\mathrm{H} 206$ & 0.9400 & C6-H6B & 0.9700 \\
\hline $\mathrm{C} 207-\mathrm{C} 212$ & $1.380(7)$ & $\mathrm{C} 6-\mathrm{H} 6 \mathrm{C}$ & 0.9700 \\
\hline $\mathrm{C} 4-\mathrm{Ir} 1-\mathrm{C} 1$ & $89.27(17)$ & $\mathrm{C} 204-\mathrm{C} 205-\mathrm{C} 206$ & $119.6(6)$ \\
\hline $\mathrm{C} 4-\mathrm{Ir} 1-\mathrm{P} 4$ & $89.08(12)$ & $\mathrm{C} 204-\mathrm{C} 205-\mathrm{H} 205$ & 120.2 \\
\hline $\mathrm{C} 1-\mathrm{Ir} 1-\mathrm{P} 4$ & $84.94(12)$ & $\mathrm{C} 206-\mathrm{C} 205-\mathrm{H} 205$ & 120.2 \\
\hline $\mathrm{C} 4-\mathrm{Ir} 1-\mathrm{P} 1$ & $94.22(12)$ & $\mathrm{C} 201-\mathrm{C} 206-\mathrm{C} 205$ & $120.5(6)$ \\
\hline $\mathrm{C} 1-\mathrm{Ir} 1-\mathrm{P} 1$ & $89.10(12)$ & $\mathrm{C} 201-\mathrm{C} 206-\mathrm{H} 206$ & 119.7 \\
\hline $\mathrm{P} 4-\mathrm{Ir} 1-\mathrm{P} 1$ & $173.16(4)$ & $\mathrm{C} 205-\mathrm{C} 206-\mathrm{H} 206$ & 119.7 \\
\hline $\mathrm{C} 4-\mathrm{Ir} 1-\mathrm{Cl1}$ & $94.42(13)$ & $\mathrm{C} 212-\mathrm{C} 207-\mathrm{C} 208$ & $118.7(5)$ \\
\hline $\mathrm{C} 1-\mathrm{Ir} 1-\mathrm{Cl} 1$ & $175.28(12)$ & $\mathrm{C} 212-\mathrm{C} 207-\mathrm{P} 2$ & $123.2(4)$ \\
\hline $\mathrm{P} 4-\mathrm{Ir} 1-\mathrm{Cl} 1$ & $92.17(4)$ & $\mathrm{C} 208-\mathrm{C} 207-\mathrm{P} 2$ & $118.1(4)$ \\
\hline $\mathrm{P} 1-\mathrm{Ir} 1-\mathrm{Cl} 1$ & $93.55(4)$ & $\mathrm{C} 209-\mathrm{C} 208-\mathrm{C} 207$ & $120.3(6)$ \\
\hline $\mathrm{C} 4-\mathrm{Ir} 1-\mathrm{H} 1$ & $176.6(13)$ & $\mathrm{C} 209-\mathrm{C} 208-\mathrm{H} 208$ & 119.8 \\
\hline $\mathrm{C} 1-\mathrm{Ir} 1-\mathrm{H} 1$ & $87.8(14)$ & C207-C208-H208 & 119.8 \\
\hline $\mathrm{P} 4-\mathrm{Ir} 1-\mathrm{H} 1$ & $89.0(13)$ & $\mathrm{C} 210-\mathrm{C} 209-\mathrm{C} 208$ & $120.7(7)$ \\
\hline $\mathrm{P} 1-\mathrm{Ir} 1-\mathrm{H} 1$ & $87.4(13)$ & C210-C209-H209 & 119.7 \\
\hline $\mathrm{C} 11-\mathrm{Ir} 1-\mathrm{H} 1$ & $88.4(14)$ & C208-C209-H209 & 119.7 \\
\hline $\mathrm{C} 107-\mathrm{P} 1-\mathrm{C} 101$ & $104.0(2)$ & $\mathrm{C} 209-\mathrm{C} 210-\mathrm{C} 211$ & $120.0(6)$ \\
\hline $\mathrm{C} 107-\mathrm{P} 1-\mathrm{C} 2$ & $104.7(2)$ & $\mathrm{C} 209-\mathrm{C} 210-\mathrm{H} 210$ & 120.0 \\
\hline $\mathrm{C} 101-\mathrm{P} 1-\mathrm{C} 2$ & $103.4(2)$ & $\mathrm{C} 211-\mathrm{C} 210-\mathrm{H} 210$ & 120.0 \\
\hline C107-P1-Ir1 & $117.25(15)$ & $\mathrm{C} 210-\mathrm{C} 211-\mathrm{C} 212$ & $120.7(6)$ \\
\hline $\mathrm{C} 101-\mathrm{P} 1-\mathrm{Ir} 1$ & $119.28(16)$ & $\mathrm{C} 210-\mathrm{C} 211-\mathrm{H} 211$ & 119.7 \\
\hline $\mathrm{C} 2-\mathrm{P} 1-\mathrm{Ir} 1$ & $106.46(14)$ & $\mathrm{C} 212-\mathrm{C} 211-\mathrm{H} 211$ & 119.7 \\
\hline $\mathrm{C} 1-\mathrm{P} 2-\mathrm{C} 201$ & $116.7(2)$ & $\mathrm{C} 207-\mathrm{C} 212-\mathrm{C} 211$ & $119.6(6)$ \\
\hline $\mathrm{C} 1-\mathrm{P} 2-\mathrm{C} 207$ & $114.7(2)$ & $\mathrm{C} 207-\mathrm{C} 212-\mathrm{H} 212$ & 120.2 \\
\hline $\mathrm{C} 201-\mathrm{P} 2-\mathrm{C} 207$ & $104.9(2)$ & $\mathrm{C} 211-\mathrm{C} 212-\mathrm{H} 212$ & 120.2 \\
\hline $\mathrm{C} 1-\mathrm{P} 2-\mathrm{C} 2$ & $106.8(2)$ & $\mathrm{C} 302-\mathrm{C} 301-\mathrm{C} 306$ & $118.6(4)$ \\
\hline $\mathrm{C} 201-\mathrm{P} 2-\mathrm{C} 2$ & $108.4(2)$ & $\mathrm{C} 302-\mathrm{C} 301-\mathrm{P} 3$ & $121.4(4)$ \\
\hline $\mathrm{C} 207-\mathrm{P} 2-\mathrm{C} 2$ & $104.6(2)$ & $\mathrm{C} 306-\mathrm{C} 301-\mathrm{P} 3$ & $119.7(4)$ \\
\hline $\mathrm{C} 1-\mathrm{P} 3-\mathrm{C} 301$ & $120.2(2)$ & $\mathrm{C} 301-\mathrm{C} 302-\mathrm{C} 303$ & $120.0(5)$ \\
\hline $\mathrm{C} 1-\mathrm{P} 3-\mathrm{C} 307$ & $114.8(2)$ & $\mathrm{C} 301-\mathrm{C} 302-\mathrm{H} 302$ & 120.0 \\
\hline $\mathrm{C} 301-\mathrm{P} 3-\mathrm{C} 307$ & $103.7(2)$ & $\mathrm{C} 303-\mathrm{C} 302-\mathrm{H} 302$ & 120.0 \\
\hline $\mathrm{C} 1-\mathrm{P} 3-\mathrm{C} 3$ & $106.6(2)$ & $\mathrm{C} 304-\mathrm{C} 303-\mathrm{C} 302$ & $120.7(5)$ \\
\hline $\mathrm{C} 301-\mathrm{P} 3-\mathrm{C} 3$ & $101.9(2)$ & $\mathrm{C} 304-\mathrm{C} 303-\mathrm{H} 303$ & 119.7 \\
\hline
\end{tabular}




\begin{tabular}{|c|c|}
\hline $\mathrm{C} 307-\mathrm{P} 3-\mathrm{C} 3$ & $108.6(2)$ \\
\hline $\mathrm{C} 401-\mathrm{P} 4-\mathrm{C} 407$ & $101.6(2)$ \\
\hline $\mathrm{C} 401-\mathrm{P} 4-\mathrm{C} 3$ & $104.2(2)$ \\
\hline $\mathrm{C} 407-\mathrm{P} 4-\mathrm{C} 3$ & $103.4(2)$ \\
\hline $\mathrm{C} 401-\mathrm{P} 4-\mathrm{Ir} 1$ & $118.52(15)$ \\
\hline $\mathrm{C} 407-\mathrm{P} 4-\mathrm{Ir} 1$ & $120.92(17)$ \\
\hline $\mathrm{C} 3-\mathrm{P} 4-\mathrm{Ir} 1$ & $106.10(15)$ \\
\hline $\mathrm{N} 1-\mathrm{C} 4-\mathrm{Ir} 1$ & $175.0(4)$ \\
\hline $\mathrm{P} 3-\mathrm{C} 1-\mathrm{P} 2$ & $127.8(3)$ \\
\hline $\mathrm{P} 3-\mathrm{C} 1-\mathrm{Ir} 1$ & $119.7(2)$ \\
\hline $\mathrm{P} 2-\mathrm{C} 1-\mathrm{Ir} 1$ & $112.5(2)$ \\
\hline $\mathrm{P} 2-\mathrm{C} 2-\mathrm{P} 1$ & $105.9(2)$ \\
\hline $\mathrm{P} 2-\mathrm{C} 2-\mathrm{H} 2 \mathrm{~A}$ & 110.6 \\
\hline $\mathrm{P} 1-\mathrm{C} 2-\mathrm{H} 2 \mathrm{~A}$ & 110.6 \\
\hline $\mathrm{P} 2-\mathrm{C} 2-\mathrm{H} 2 \mathrm{~B}$ & 110.6 \\
\hline $\mathrm{P} 1-\mathrm{C} 2-\mathrm{H} 2 \mathrm{~B}$ & 110.6 \\
\hline $\mathrm{H} 2 \mathrm{~A}-\mathrm{C} 2-\mathrm{H} 2 \mathrm{~B}$ & 108.7 \\
\hline $\mathrm{P} 3-\mathrm{C} 3-\mathrm{P} 4$ & $110.8(2)$ \\
\hline $\mathrm{P} 3-\mathrm{C} 3-\mathrm{H} 3 \mathrm{~A}$ & 109.5 \\
\hline $\mathrm{P} 4-\mathrm{C} 3-\mathrm{H} 3 \mathrm{~A}$ & 109.5 \\
\hline $\mathrm{P} 3-\mathrm{C} 3-\mathrm{H} 3 \mathrm{~B}$ & 109.5 \\
\hline $\mathrm{P} 4-\mathrm{C} 3-\mathrm{H} 3 \mathrm{~B}$ & 109.5 \\
\hline $\mathrm{H} 3 \mathrm{~A}-\mathrm{C} 3-\mathrm{H} 3 \mathrm{~B}$ & 108.1 \\
\hline $\mathrm{C} 102-\mathrm{C} 101-\mathrm{C} 106$ & $119.0(4)$ \\
\hline $\mathrm{C} 102-\mathrm{C} 101-\mathrm{P} 1$ & $118.7(4)$ \\
\hline $\mathrm{C} 106-\mathrm{C} 101-\mathrm{P} 1$ & $122.2(4)$ \\
\hline $\mathrm{C} 103-\mathrm{C} 102-\mathrm{C} 101$ & $119.8(5)$ \\
\hline $\mathrm{C} 103-\mathrm{C} 102-\mathrm{H} 102$ & 120.1 \\
\hline $\mathrm{C} 101-\mathrm{C} 102-\mathrm{H} 102$ & 120.1 \\
\hline $\mathrm{C} 104-\mathrm{C} 103-\mathrm{C} 102$ & $120.8(5)$ \\
\hline $\mathrm{C} 104-\mathrm{C} 103-\mathrm{H} 103$ & 119.6 \\
\hline $\mathrm{C} 102-\mathrm{C} 103-\mathrm{H} 103$ & 119.6 \\
\hline $\mathrm{C} 105-\mathrm{C} 104-\mathrm{C} 103$ & $119.9(5)$ \\
\hline $\mathrm{C} 105-\mathrm{C} 104-\mathrm{H} 104$ & 120.1 \\
\hline $\mathrm{C} 103-\mathrm{C} 104-\mathrm{H} 104$ & 120.1 \\
\hline $\mathrm{C} 104-\mathrm{C} 105-\mathrm{C} 106$ & $120.8(5)$ \\
\hline $\mathrm{C} 104-\mathrm{C} 105-\mathrm{H} 105$ & 119.6 \\
\hline $\mathrm{C} 106-\mathrm{C} 105-\mathrm{H} 105$ & 119.6 \\
\hline $\mathrm{C} 105-\mathrm{C} 106-\mathrm{C} 101$ & $119.6(5)$ \\
\hline $\mathrm{C} 105-\mathrm{C} 106-\mathrm{H} 106$ & 120.2 \\
\hline $\mathrm{C} 101-\mathrm{C} 106-\mathrm{H} 106$ & 120.2 \\
\hline $\mathrm{C} 108-\mathrm{C} 107-\mathrm{C} 112$ & $117.8(4)$ \\
\hline $\mathrm{C} 108-\mathrm{C} 107-\mathrm{P} 1$ & $119.9(4)$ \\
\hline $\mathrm{C} 112-\mathrm{C} 107-\mathrm{P} 1$ & $122.3(4)$ \\
\hline $\mathrm{C} 109-\mathrm{C} 108-\mathrm{C} 107$ & $121.5(5)$ \\
\hline $\mathrm{C} 109-\mathrm{C} 108-\mathrm{H} 108$ & 119.2 \\
\hline $\mathrm{C} 107-\mathrm{C} 108-\mathrm{H} 108$ & 119.2 \\
\hline $\mathrm{C} 110-\mathrm{C} 109-\mathrm{C} 108$ & $119.8(5)$ \\
\hline
\end{tabular}

$\begin{array}{ll}\mathrm{C} 302-\mathrm{C} 303-\mathrm{H} 303 & 119.7 \\ \mathrm{C} 303-\mathrm{C} 304-\mathrm{C} 305 & 119.4(5) \\ \mathrm{C} 303-\mathrm{C} 304-\mathrm{H} 304 & 120.3 \\ \mathrm{C} 305-\mathrm{C} 304-\mathrm{H} 304 & 120.3 \\ \mathrm{C} 306-\mathrm{C} 305-\mathrm{C} 304 & 120.4(5) \\ \mathrm{C} 306-\mathrm{C} 305-\mathrm{H} 305 & 119.8 \\ \mathrm{C} 304-\mathrm{C} 305-\mathrm{H} 305 & 119.8 \\ \mathrm{C} 305-\mathrm{C} 306-\mathrm{C} 301 & 120.8(5) \\ \mathrm{C} 305-\mathrm{C} 306-\mathrm{H} 306 & 119.6 \\ \mathrm{C} 301-\mathrm{C} 306-\mathrm{H} 306 & 119.6 \\ \mathrm{C} 308-\mathrm{C} 307-\mathrm{C} 312 & 118.3(5) \\ \mathrm{C} 308-\mathrm{C} 307-\mathrm{P} 3 & 118.9(4) \\ \mathrm{C} 312-\mathrm{C} 307-\mathrm{P} 3 & 122.7(4) \\ \mathrm{C} 307-\mathrm{C} 308-\mathrm{C} 309 & 121.0(5) \\ \mathrm{C} 307-\mathrm{C} 308-\mathrm{H} 308 & 119.5\end{array}$

$\mathrm{C} 307-\mathrm{C} 308-\mathrm{H} 308 \quad 119.5$

$\mathrm{C} 309-\mathrm{C} 308-\mathrm{H} 308 \quad 119.5$

$\mathrm{C} 310-\mathrm{C} 309-\mathrm{C} 308 \quad 120.1(6)$

C310-C309-H309 119.9

C308-C309-H309 119.9

$\mathrm{C} 309-\mathrm{C} 310-\mathrm{C} 311 \quad 120.2(6)$

$\mathrm{C} 309-\mathrm{C} 310-\mathrm{H} 310 \quad 119.9$

$\mathrm{C} 311-\mathrm{C} 310-\mathrm{H} 310 \quad 119.9$

$\mathrm{C} 310-\mathrm{C} 311-\mathrm{C} 312 \quad 119.9(6)$

C310-C311-H311 120.1

$\mathrm{C} 312-\mathrm{C} 311-\mathrm{H} 311 \quad 120.1$

C307-C312-C311 120.5 (5)

$\mathrm{C} 307-\mathrm{C} 312-\mathrm{H} 312 \quad 119.7$

$\mathrm{C} 311-\mathrm{C} 312-\mathrm{H} 312$

$\mathrm{C} 406-\mathrm{C} 401-\mathrm{C} 402 \quad 117.7$ (5)

$\mathrm{C} 406-\mathrm{C} 401-\mathrm{P} 4 \quad 120.1$ (4)

$\mathrm{C} 402-\mathrm{C} 401-\mathrm{P} 4 \quad 121.9$ (4)

$\mathrm{C} 401-\mathrm{C} 402-\mathrm{C} 403 \quad 122.2(6)$

$\mathrm{C} 401-\mathrm{C} 402-\mathrm{H} 402$

$\mathrm{C} 403-\mathrm{C} 402-\mathrm{H} 402 \quad 118.9$

$\mathrm{C} 404-\mathrm{C} 403-\mathrm{C} 402 \quad 119.7$ (7)

$\mathrm{C} 404-\mathrm{C} 403-\mathrm{H} 403 \quad 120.1$

$\mathrm{C} 402-\mathrm{C} 403-\mathrm{H} 403 \quad 120.1$

$\mathrm{C} 405-\mathrm{C} 404-\mathrm{C} 403 \quad 120.1(6)$

$\mathrm{C} 405-\mathrm{C} 404-\mathrm{H} 404 \quad 119.9$

$\mathrm{C} 403-\mathrm{C} 404-\mathrm{H} 404$

$\mathrm{C} 404-\mathrm{C} 405-\mathrm{C} 406 \quad 120.6$ (6)

$\mathrm{C} 404-\mathrm{C} 405-\mathrm{H} 405 \quad 119.7$

$\mathrm{C} 406-\mathrm{C} 405-\mathrm{H} 405 \quad 119.7$

$\mathrm{C} 401-\mathrm{C} 406-\mathrm{C} 405 \quad 119.6$ (6)

$\mathrm{C} 401-\mathrm{C} 406-\mathrm{H} 406 \quad 120.2$

$\mathrm{C} 405-\mathrm{C} 406-\mathrm{H} 406 \quad 120.2$

$\mathrm{C} 408-\mathrm{C} 407-\mathrm{C} 412 \quad 118.2(5)$

C408-C407-P4 121.6 (4) 


\begin{tabular}{|c|c|c|c|}
\hline $\mathrm{C} 110-\mathrm{C} 109-\mathrm{H} 109$ & 120.1 & $\mathrm{C} 412-\mathrm{C} 407-\mathrm{P} 4$ & $120.2(4)$ \\
\hline $\mathrm{C} 108-\mathrm{C} 109-\mathrm{H} 109$ & 120.1 & $\mathrm{C} 407-\mathrm{C} 408-\mathrm{C} 409$ & $121.0(5)$ \\
\hline $\mathrm{C} 109-\mathrm{C} 110-\mathrm{C} 111$ & $120.0(5)$ & $\mathrm{C} 407-\mathrm{C} 408-\mathrm{H} 408$ & 119.5 \\
\hline $\mathrm{C} 109-\mathrm{C} 110-\mathrm{H} 110$ & 120.0 & $\mathrm{C} 409-\mathrm{C} 408-\mathrm{H} 408$ & 119.5 \\
\hline $\mathrm{C} 111-\mathrm{C} 110-\mathrm{H} 110$ & 120.0 & $\mathrm{C} 410-\mathrm{C} 409-\mathrm{C} 408$ & $120.2(6)$ \\
\hline $\mathrm{C} 110-\mathrm{C} 111-\mathrm{C} 112$ & $120.5(5)$ & $\mathrm{C} 410-\mathrm{C} 409-\mathrm{H} 409$ & 119.9 \\
\hline $\mathrm{C} 110-\mathrm{C} 111-\mathrm{H} 111$ & 119.7 & $\mathrm{C} 408-\mathrm{C} 409-\mathrm{H} 409$ & 119.9 \\
\hline $\mathrm{C} 112-\mathrm{C} 111-\mathrm{H} 111$ & 119.7 & $\mathrm{C} 411-\mathrm{C} 410-\mathrm{C} 409$ & $119.6(6)$ \\
\hline $\mathrm{C} 111-\mathrm{C} 112-\mathrm{C} 107$ & $120.2(5)$ & $\mathrm{C} 411-\mathrm{C} 410-\mathrm{H} 410$ & 120.2 \\
\hline $\mathrm{C} 111-\mathrm{C} 112-\mathrm{H} 112$ & 119.9 & $\mathrm{C} 409-\mathrm{C} 410-\mathrm{H} 410$ & 120.2 \\
\hline $\mathrm{C} 107-\mathrm{C} 112-\mathrm{H} 112$ & 119.9 & $\mathrm{C} 410-\mathrm{C} 411-\mathrm{C} 412$ & $121.1(6)$ \\
\hline $\mathrm{C} 202-\mathrm{C} 201-\mathrm{C} 206$ & $118.6(5)$ & $\mathrm{C} 410-\mathrm{C} 411-\mathrm{H} 411$ & 119.4 \\
\hline $\mathrm{C} 202-\mathrm{C} 201-\mathrm{P} 2$ & $122.0(4)$ & $\mathrm{C} 412-\mathrm{C} 411-\mathrm{H} 411$ & 119.4 \\
\hline $\mathrm{C} 206-\mathrm{C} 201-\mathrm{P} 2$ & $119.4(4)$ & $\mathrm{C} 411-\mathrm{C} 412-\mathrm{C} 407$ & $119.8(6)$ \\
\hline $\mathrm{C} 201-\mathrm{C} 202-\mathrm{C} 203$ & $120.5(6)$ & $\mathrm{C} 411-\mathrm{C} 412-\mathrm{H} 412$ & 120.1 \\
\hline $\mathrm{C} 201-\mathrm{C} 202-\mathrm{H} 202$ & 119.7 & $\mathrm{C} 407-\mathrm{C} 412-\mathrm{H} 412$ & 120.1 \\
\hline $\mathrm{C} 203-\mathrm{C} 202-\mathrm{H} 202$ & 119.7 & $\mathrm{~N} 2-\mathrm{C} 5-\mathrm{C} 6$ & $177.6(10)$ \\
\hline $\mathrm{C} 204-\mathrm{C} 203-\mathrm{C} 202$ & $119.9(6)$ & $\mathrm{C} 5-\mathrm{C} 6-\mathrm{H} 6 \mathrm{~A}$ & 109.5 \\
\hline $\mathrm{C} 204-\mathrm{C} 203-\mathrm{H} 203$ & 120.1 & $\mathrm{C} 5-\mathrm{C} 6-\mathrm{H} 6 \mathrm{~B}$ & 109.5 \\
\hline $\mathrm{C} 202-\mathrm{C} 203-\mathrm{H} 203$ & 120.1 & $\mathrm{H} 6 \mathrm{~A}-\mathrm{C} 6-\mathrm{H} 6 \mathrm{~B}$ & 109.5 \\
\hline $\mathrm{C} 203-\mathrm{C} 204-\mathrm{C} 205$ & $120.8(6)$ & $\mathrm{C} 5-\mathrm{C} 6-\mathrm{H} 6 \mathrm{C}$ & 109.5 \\
\hline $\mathrm{C} 203-\mathrm{C} 204-\mathrm{H} 204$ & 119.6 & $\mathrm{H} 6 \mathrm{~A}-\mathrm{C} 6-\mathrm{H} 6 \mathrm{C}$ & 109.5 \\
\hline $\mathrm{C} 205-\mathrm{C} 204-\mathrm{H} 204$ & 119.6 & $\mathrm{H} 6 \mathrm{~B}-\mathrm{C} 6-\mathrm{H} 6 \mathrm{C}$ & 109.5 \\
\hline $\mathrm{C} 301-\mathrm{P} 3-\mathrm{C} 1-\mathrm{P} 2$ & $76.6(4)$ & $\mathrm{C} 2-\mathrm{P} 2-\mathrm{C} 207-\mathrm{C} 212$ & $42.9(5)$ \\
\hline $\mathrm{C} 307-\mathrm{P} 3-\mathrm{C} 1-\mathrm{P} 2$ & $-48.1(4)$ & $\mathrm{C} 1-\mathrm{P} 2-\mathrm{C} 207-\mathrm{C} 208$ & $-21.3(5)$ \\
\hline $\mathrm{C} 3-\mathrm{P} 3-\mathrm{C} 1-\mathrm{P} 2$ & $-168.3(3)$ & $\mathrm{C} 201-\mathrm{P} 2-\mathrm{C} 207-\mathrm{C} 208$ & $108.0(4)$ \\
\hline $\mathrm{C} 301-\mathrm{P} 3-\mathrm{C} 1-\mathrm{Ir} 1$ & $-101.9(3)$ & $\mathrm{C} 2-\mathrm{P} 2-\mathrm{C} 207-\mathrm{C} 208$ & $-138.0(4)$ \\
\hline C307-P3-C1-Ir1 & $133.4(2)$ & $\mathrm{C} 212-\mathrm{C} 207-\mathrm{C} 208-\mathrm{C} 209$ & $0.7(8)$ \\
\hline $\mathrm{C} 3-\mathrm{P} 3-\mathrm{C} 1-\mathrm{Ir} 1$ & $13.1(3)$ & $\mathrm{P} 2-\mathrm{C} 207-\mathrm{C} 208-\mathrm{C} 209$ & $-178.4(4)$ \\
\hline $\mathrm{C} 201-\mathrm{P} 2-\mathrm{C} 1-\mathrm{P} 3$ & $-13.5(4)$ & $\mathrm{C} 207-\mathrm{C} 208-\mathrm{C} 209-\mathrm{C} 210$ & $-1.7(9)$ \\
\hline $\mathrm{C} 207-\mathrm{P} 2-\mathrm{C} 1-\mathrm{P} 3$ & $109.7(3)$ & $\mathrm{C} 208-\mathrm{C} 209-\mathrm{C} 210-\mathrm{C} 211$ & $1.9(10)$ \\
\hline $\mathrm{C} 2-\mathrm{P} 2-\mathrm{C} 1-\mathrm{P} 3$ & $-134.9(3)$ & $\mathrm{C} 209-\mathrm{C} 210-\mathrm{C} 211-\mathrm{C} 212$ & $-1.2(10)$ \\
\hline $\mathrm{C} 201-\mathrm{P} 2-\mathrm{C} 1-\mathrm{Ir} 1$ & $165.1(2)$ & $\mathrm{C} 208-\mathrm{C} 207-\mathrm{C} 212-\mathrm{C} 211$ & $-0.1(8)$ \\
\hline $\mathrm{C} 207-\mathrm{P} 2-\mathrm{C} 1-\mathrm{Ir} 1$ & $-71.7(3)$ & $\mathrm{P} 2-\mathrm{C} 207-\mathrm{C} 212-\mathrm{C} 211$ & $179.0(4)$ \\
\hline $\mathrm{C} 2-\mathrm{P} 2-\mathrm{C} 1-\mathrm{Ir} 1$ & $43.7(3)$ & $\mathrm{C} 210-\mathrm{C} 211-\mathrm{C} 212-\mathrm{C} 207$ & $0.3(9)$ \\
\hline $\mathrm{C} 1-\mathrm{P} 2-\mathrm{C} 2-\mathrm{P} 1$ & $-45.5(3)$ & $\mathrm{C} 1-\mathrm{P} 3-\mathrm{C} 301-\mathrm{C} 302$ & $-1.3(5)$ \\
\hline $\mathrm{C} 201-\mathrm{P} 2-\mathrm{C} 2-\mathrm{P} 1$ & $-172.0(2)$ & $\mathrm{C} 307-\mathrm{P} 3-\mathrm{C} 301-\mathrm{C} 302$ & $128.6(4)$ \\
\hline $\mathrm{C} 207-\mathrm{P} 2-\mathrm{C} 2-\mathrm{P} 1$ & $76.5(3)$ & $\mathrm{C} 3-\mathrm{P} 3-\mathrm{C} 301-\mathrm{C} 302$ & $-118.7(4)$ \\
\hline $\mathrm{C} 107-\mathrm{P} 1-\mathrm{C} 2-\mathrm{P} 2$ & $-96.5(2)$ & $\mathrm{C} 1-\mathrm{P} 3-\mathrm{C} 301-\mathrm{C} 306$ & $171.8(4)$ \\
\hline $\mathrm{C} 101-\mathrm{P} 1-\mathrm{C} 2-\mathrm{P} 2$ & $154.8(2)$ & C307-P3-C301-C306 & $-58.3(4)$ \\
\hline $\mathrm{Ir} 1-\mathrm{P} 1-\mathrm{C} 2-\mathrm{P} 2$ & $28.3(2)$ & $\mathrm{C} 3-\mathrm{P} 3-\mathrm{C} 301-\mathrm{C} 306$ & $54.4(4)$ \\
\hline $\mathrm{C} 1-\mathrm{P} 3-\mathrm{C} 3-\mathrm{P} 4$ & $13.2(3)$ & $\mathrm{C} 306-\mathrm{C} 301-\mathrm{C} 302-\mathrm{C} 303$ & $3.4(8)$ \\
\hline $\mathrm{C} 301-\mathrm{P} 3-\mathrm{C} 3-\mathrm{P} 4$ & $140.1(2)$ & $\mathrm{P} 3-\mathrm{C} 301-\mathrm{C} 302-\mathrm{C} 303$ & $176.6(4)$ \\
\hline $\mathrm{C} 307-\mathrm{P} 3-\mathrm{C} 3-\mathrm{P} 4$ & $-110.9(3)$ & $\mathrm{C} 301-\mathrm{C} 302-\mathrm{C} 303-\mathrm{C} 304$ & $-1.0(8)$ \\
\hline $\mathrm{C} 401-\mathrm{P} 4-\mathrm{C} 3-\mathrm{P} 3$ & $-156.9(2)$ & $\mathrm{C} 302-\mathrm{C} 303-\mathrm{C} 304-\mathrm{C} 305$ & $-1.4(8)$ \\
\hline $\mathrm{C} 407-\mathrm{P} 4-\mathrm{C} 3-\mathrm{P} 3$ & $97.2(3)$ & $\mathrm{C} 303-\mathrm{C} 304-\mathrm{C} 305-\mathrm{C} 306$ & $1.3(9)$ \\
\hline $\mathrm{Ir} 1-\mathrm{P} 4-\mathrm{C} 3-\mathrm{P} 3$ & $-31.1(3)$ & $\mathrm{C} 304-\mathrm{C} 305-\mathrm{C} 306-\mathrm{C} 301$ & $1.2(8)$ \\
\hline
\end{tabular}




$\begin{array}{ll}\text { C107-P1-C101-C102 } & 98.8(4) \\ \text { C2-P1-C101-C102 } & -152.1(4) \\ \text { Ir1-P1-C101-C102 } & -34.2(4) \\ \text { C107-P1-C101-C106 } & -82.2(4) \\ \text { C2-P1-C101-C106 } & 27.0(4) \\ \text { Ir1-P1-C101-C106 } & 144.9(3) \\ \text { C106-C101-C102-C103 } & 0.5(7) \\ \text { P1-C101-C102-C103 } & 179.6(4) \\ \text { C101-C102-C103-C104 } & -1.2(9) \\ \text { C102-C103-C104-C105 } & 0.8(9) \\ \text { C103-C104-C105-C106 } & 0.4(9) \\ \text { C104-C105-C106-C101 } & -1.1(8) \\ \text { C102-C101-C106-C105 } & 0.6(7) \\ \text { P1-C101-C106-C105 } & -178.5(4) \\ \text { C101-P1-C107-C108 } & -121.7(4) \\ \text { C2-P1-C107-C108 } & 130.1(4) \\ \text { Ir1-P1-C107-C108 } & 12.4(4) \\ \text { C101-P1-C107-C112 } & 59.7(4) \\ \text { C2-P1-C107-C112 } & -48.5(4) \\ \text { Ir1-P1-C107-C112 } & -166.2(3) \\ \text { C112-C107-C108-C109 } & 1.4(7) \\ \text { P1-C107-C108-C109 } & -177.2(4) \\ \text { C107-C108-C109-C110 } & 0.8(8) \\ \text { C108-C109-C110-C111 } & -2.8(9) \\ \text { C109-C110-C111-C112 } & 2.5(8) \\ \text { C110-C111-C112-C107 } & -0.2(8) \\ \text { C108-C107-C112-C111 } & -1.7(7) \\ \text { P1-C107-C112-C111 } & 176.9(4) \\ \text { C1-P2-C201-C202 } & -84.0(5) \\ \text { C207-P2-C201-C202 } & 147.9(4) \\ \text { C2-P2-C201-C202 } & 36.6(5) \\ \text { C1-P2-C201-C206 } & 93.2(5) \\ \text { C207-P2-C201-C206 } & -34.9(5) \\ \text { C2-P2-C201-C206 } & -146.2(4) \\ \text { C206-C201-C202-C203 } & -1.8(8) \\ \text { P2-C201-C202-C203 } & 175.4(4) \\ \text { C201-C202-C203-C204 } & 0.4(9) \\ \text { C202-C203-C204-C205 } & 1.1(10) \\ \text { C203-C204-C205-C206 } & -1.1(10) \\ \text { C202-C201-C206-C205 } & 1.7(8) \\ \text { P2-C201-C206-C205 } & -175.6(5) \\ \text { C204-C205-C206-C201 } & -0.2(9) \\ \text { C1-P2-C207-C212 } & 159.6(4) \\ \text { C201-P2-C207-C212 } & -71.0(5) \\ & \end{array}$

\begin{tabular}{|c|c|}
\hline $\mathrm{C} 302-\mathrm{C} 301-\mathrm{C} 306-\mathrm{C} 305$ & $-3.6(8)$ \\
\hline P3-C301-C306-C305 & $-176.9(4)$ \\
\hline $\mathrm{C} 1-\mathrm{P} 3-\mathrm{C} 307-\mathrm{C} 308$ & $-65.0(5)$ \\
\hline $\mathrm{C} 301-\mathrm{P} 3-\mathrm{C} 307-\mathrm{C} 308$ & $161.9(4)$ \\
\hline $\mathrm{C} 3-\mathrm{P} 3-\mathrm{C} 307-\mathrm{C} 308$ & $54.1(5)$ \\
\hline $\mathrm{C} 1-\mathrm{P} 3-\mathrm{C} 307-\mathrm{C} 312$ & $110.4(4)$ \\
\hline $\mathrm{C} 301-\mathrm{P} 3-\mathrm{C} 307-\mathrm{C} 312$ & $-22.6(5)$ \\
\hline $\mathrm{C} 3-\mathrm{P} 3-\mathrm{C} 307-\mathrm{C} 312$ & $-130.4(4)$ \\
\hline C312-C307-C308-C309 & $-0.4(9)$ \\
\hline P3-C307-C308-C309 & $175.2(5)$ \\
\hline $\mathrm{C} 307-\mathrm{C} 308-\mathrm{C} 309-\mathrm{C} 310$ & $-0.3(11)$ \\
\hline $\mathrm{C} 308-\mathrm{C} 309-\mathrm{C} 310-\mathrm{C} 311$ & $1.0(12)$ \\
\hline $\mathrm{C} 309-\mathrm{C} 310-\mathrm{C} 311-\mathrm{C} 312$ & $-1.0(11)$ \\
\hline $\mathrm{C} 308-\mathrm{C} 307-\mathrm{C} 312-\mathrm{C} 311$ & $0.4(8)$ \\
\hline $\mathrm{P} 3-\mathrm{C} 307-\mathrm{C} 312-\mathrm{C} 311$ & $-175.1(5)$ \\
\hline $\mathrm{C} 310-\mathrm{C} 311-\mathrm{C} 312-\mathrm{C} 307$ & $0.3(10)$ \\
\hline $\mathrm{C} 407-\mathrm{P} 4-\mathrm{C} 401-\mathrm{C} 406$ & $-38.3(5)$ \\
\hline $\mathrm{C} 3-\mathrm{P} 4-\mathrm{C} 401-\mathrm{C} 406$ & $-145.5(4)$ \\
\hline $\mathrm{Ir} 1-\mathrm{P} 4-\mathrm{C} 401-\mathrm{C} 406$ & $96.9(4)$ \\
\hline $\mathrm{C} 407-\mathrm{P} 4-\mathrm{C} 401-\mathrm{C} 402$ & $148.4(4)$ \\
\hline $\mathrm{C} 3-\mathrm{P} 4-\mathrm{C} 401-\mathrm{C} 402$ & $41.2(5)$ \\
\hline $\mathrm{Ir} 1-\mathrm{P} 4-\mathrm{C} 401-\mathrm{C} 402$ & $-76.4(4)$ \\
\hline $\mathrm{C} 406-\mathrm{C} 401-\mathrm{C} 402-\mathrm{C} 403$ & $2.6(8)$ \\
\hline P4-C401-C402-C403 & $176.1(4)$ \\
\hline $\mathrm{C} 401-\mathrm{C} 402-\mathrm{C} 403-\mathrm{C} 404$ & $-0.4(9)$ \\
\hline $\mathrm{C} 402-\mathrm{C} 403-\mathrm{C} 404-\mathrm{C} 405$ & $-2.0(10)$ \\
\hline $\mathrm{C} 403-\mathrm{C} 404-\mathrm{C} 405-\mathrm{C} 406$ & $2.0(10)$ \\
\hline $\mathrm{C} 402-\mathrm{C} 401-\mathrm{C} 406-\mathrm{C} 405$ & $-2.5(8)$ \\
\hline $\mathrm{P} 4-\mathrm{C} 401-\mathrm{C} 406-\mathrm{C} 405$ & $-176.1(4)$ \\
\hline $\mathrm{C} 404-\mathrm{C} 405-\mathrm{C} 406-\mathrm{C} 401$ & $0.3(9)$ \\
\hline $\mathrm{C} 401-\mathrm{P} 4-\mathrm{C} 407-\mathrm{C} 408$ & $123.2(5)$ \\
\hline $\mathrm{C} 3-\mathrm{P} 4-\mathrm{C} 407-\mathrm{C} 408$ & $-129.0(5)$ \\
\hline Ir1-P4-C407-C408 & $-10.6(5)$ \\
\hline $\mathrm{C} 401-\mathrm{P} 4-\mathrm{C} 407-\mathrm{C} 412$ & $-57.8(5)$ \\
\hline $\mathrm{C} 3-\mathrm{P} 4-\mathrm{C} 407-\mathrm{C} 412$ & $50.1(5)$ \\
\hline Ir1-P4-C407-C412 & $168.5(4)$ \\
\hline $\mathrm{C} 412-\mathrm{C} 407-\mathrm{C} 408-\mathrm{C} 409$ & $-0.1(9)$ \\
\hline $\mathrm{P} 4-\mathrm{C} 407-\mathrm{C} 408-\mathrm{C} 409$ & $179.0(5)$ \\
\hline $\mathrm{C} 407-\mathrm{C} 408-\mathrm{C} 409-\mathrm{C} 410$ & $-1.7(11)$ \\
\hline $\mathrm{C} 408-\mathrm{C} 409-\mathrm{C} 410-\mathrm{C} 411$ & $2.1(12)$ \\
\hline $\mathrm{C} 409-\mathrm{C} 410-\mathrm{C} 411-\mathrm{C} 412$ & $-0.6(13)$ \\
\hline $\mathrm{C} 410-\mathrm{C} 411-\mathrm{C} 412-\mathrm{C} 407$ & $-1.2(11)$ \\
\hline $\mathrm{C} 408-\mathrm{C} 407-\mathrm{C} 412-\mathrm{C} 411$ & $1.5(9)$ \\
\hline $\mathrm{P} 4-\mathrm{C} 407-\mathrm{C} 412-\mathrm{C} 411$ & $-177.6(5)$ \\
\hline
\end{tabular}


Refinement. 2:1 positional disorder of the counter anion Cl1:Cl1A. The solvent molecules $\mathrm{CH}_{2} \mathrm{Cl}_{2}$ and $\mathrm{C}_{7} \mathrm{H}_{8}$ show occupational disorder, whereas the toluene shows additional positional disorder of ratio 1:1 with some nearly overlying carbon atoms. We think, because of short intermolecular $\mathrm{Cl} \cdots \mathrm{C}$ contacts between $\mathrm{CH}_{2} \mathrm{Cl}_{2}$ and $\mathrm{C}_{7} \mathrm{H}_{8}$, that there is a correlation between these molecules. Therefore, the two solvate molecules $\mathrm{Cl} 3-\mathrm{C} 10-14$ and $\mathrm{Cl} 5-\mathrm{C} 11-\mathrm{Cl} 6$ have an occupancy of 0.75 and the 'two' toluene molecules, C12-C18 and C19-C25, an occupancy of 0.25 . Several bond restraints must be used to refine the toluene carbon atoms reasonably isotropically.

Fractional atomic coordinates and isotropic or equivalent isotropic displacement parameters $\left(\hat{A}^{2}\right)$

\begin{tabular}{|c|c|c|c|c|c|}
\hline & $x$ & $y$ & $z$ & $U_{\text {iso }} * / U_{\text {eq }}$ & Occ. $(<1)$ \\
\hline Ir1 & $0.37466(2)$ & $0.64909(2)$ & $-0.05631(2)$ & $0.03621(9)$ & \\
\hline $\mathrm{P} 1$ & $0.32978(5)$ & $0.71589(7)$ & $-0.11572(6)$ & $0.0422(3)$ & \\
\hline $\mathrm{P} 2$ & $0.35994(5)$ & $0.59865(8)$ & $-0.19050(6)$ & 0.0410 & \\
\hline P3 & $0.36065(6)$ & $0.70441(8)$ & $0.03213(6)$ & $0.0451(3)$ & \\
\hline P4 & $0.31226(5)$ & $0.59668(8)$ & $-0.01288(6)$ & $0.0437(3)$ & \\
\hline $\mathrm{Cl1}$ & $0.26332(16)$ & 0.71646 (19) & $-0.30485(14)$ & $0.0456(8)$ & 0.65 \\
\hline C11A & $0.2678(3)$ & $0.7368(3)$ & $-0.3219(2)$ & $0.0476(18)$ & 0.35 \\
\hline $\mathrm{Cl} 2$ & $0.57143(7)$ & $0.68950(13)$ & $0.14255(9)$ & $0.0891(6)$ & \\
\hline N1 & $0.44176(16)$ & $0.6172(2)$ & $-0.04574(17)$ & $0.0402(10)$ & \\
\hline $\mathrm{N} 2$ & $0.46127(17)$ & $0.5721(3)$ & $-0.08336(19)$ & $0.0470(11)$ & \\
\hline N3 & $0.43390(16)$ & $0.5618(2)$ & $-0.12734(19)$ & $0.0454(11)$ & \\
\hline $\mathrm{C} 1$ & $0.3915(2)$ & 0.5945 & $-0.1255(2)$ & $0.0405(12)$ & \\
\hline $\mathrm{C} 2$ & $0.3129(2)$ & $0.6596(3)$ & $-0.1760(2)$ & $0.0431(12)$ & \\
\hline $\mathrm{H} 2 \mathrm{~A}$ & 0.2837 & 0.6341 & -0.1670 & $0.052 *$ & \\
\hline $\mathrm{H} 2 \mathrm{~B}$ & 0.3070 & 0.6886 & -0.2095 & $0.052 *$ & \\
\hline $\mathrm{C} 3$ & $0.3010(2)$ & $0.6647(3)$ & 0.0405 & $0.0524(14)$ & \\
\hline $\mathrm{H} 3 \mathrm{~A}$ & 0.2956 & 0.6456 & 0.0784 & $0.063 *$ & \\
\hline H3B & 0.2752 & 0.6965 & 0.0299 & $0.063 *$ & \\
\hline $\mathrm{C} 4$ & 0.47418 (19) & $0.6336(3)$ & $-0.0010(2)$ & $0.0421(12)$ & \\
\hline $\mathrm{C} 5$ & $0.4929(2)$ & $0.6995(3)$ & $0.0022(3)$ & $0.0575(15)$ & \\
\hline H5 & 0.4846 & 0.7332 & -0.0250 & $0.069 *$ & \\
\hline C6 & $0.5240(2)$ & $0.7170(4)$ & 0.0453 & $0.0654(18)$ & \\
\hline H6 & 0.5373 & 0.7622 & 0.0474 & $0.078 *$ & \\
\hline $\mathrm{C} 7$ & $0.5352(2)$ & $0.6665(4)$ & 0.0852 & 0.0607 (17) & \\
\hline $\mathrm{C} 8$ & $0.5178(2)$ & $0.6007(4)$ & $0.0822(3)$ & $0.0576(16)$ & \\
\hline $\mathrm{H} 8$ & 0.5266 & 0.5669 & 0.1091 & $0.069 *$ & \\
\hline C9 & $0.4868(2)$ & 0.5838 & 0.0388 & $0.0539(15)$ & \\
\hline H9 & 0.4743 & 0.5383 & 0.0364 & $0.065^{*}$ & \\
\hline $\mathrm{C} 101$ & $0.2731(2)$ & 0.7563 & -0.0966 (2) & $0.0506(14)$ & \\
\hline $\mathrm{C} 102$ & $0.2743(3)$ & $0.8171(4)$ & $-0.0636(3)$ & 0.0679 (19) & \\
\hline H102 & 0.3034 & 0.8384 & -0.0545 & $0.081 *$ & \\
\hline $\mathrm{C} 103$ & $0.2310(4)$ & $0.8460(5)$ & -0.0439 & $0.084(3)$ & \\
\hline H103 & 0.2312 & 0.8864 & -0.0211 & $0.101^{*}$ & \\
\hline $\mathrm{C} 104$ & $0.1885(4)$ & $0.8149(6)$ & $-0.0582(4)$ & $0.094(3)$ & \\
\hline H104 & 0.1599 & 0.8339 & -0.0445 & $0.113^{*}$ & \\
\hline $\mathrm{C} 105$ & $0.1871(3)$ & $0.7572(5)$ & $-0.0918(4)$ & $0.090(3)$ & \\
\hline H105 & 0.1578 & 0.7374 & -0.1024 & $0.108 *$ & \\
\hline C106 & $0.2295(2)$ & $0.7277(4)$ & $-0.1104(3)$ & $0.0679(18)$ & \\
\hline
\end{tabular}




\begin{tabular}{|c|c|c|c|c|}
\hline H106 & 0.2284 & 0.6872 & -0.1330 & $0.081 *$ \\
\hline C107 & $0.3610(2)$ & $0.7881(3)$ & $-0.1498(2)$ & $0.0525(15)$ \\
\hline C108 & $0.4091(3)$ & $0.7953(4)$ & $-0.1443(3)$ & $0.073(2)$ \\
\hline H108 & 0.4261 & 0.7643 & -0.1209 & $0.088^{*}$ \\
\hline C109 & 0.4331 & $0.8475(5)$ & $-0.1729(5)$ & $0.107(3)$ \\
\hline H109 & 0.4661 & 0.8523 & -0.1685 & $0.129 *$ \\
\hline C110 & $0.4091(5)$ & $0.8922(5)$ & $-0.2075(5)$ & $0.113(4)$ \\
\hline H110 & 0.4255 & 0.9278 & -0.2267 & $0.136^{*}$ \\
\hline C111 & $0.3607(4)$ & $0.8854(4)$ & $-0.2143(4)$ & $0.098(3)$ \\
\hline H111 & 0.3442 & 0.9157 & -0.2388 & $0.117^{*}$ \\
\hline $\mathrm{C} 112$ & $0.3369(3)$ & $0.8348(4)$ & -0.1857 & $0.070(2)$ \\
\hline H112 & 0.3038 & 0.8309 & -0.1900 & $0.084 *$ \\
\hline C201 & $0.3333(2)$ & $0.5177(3)$ & $-0.2137(2)$ & $0.0485(13)$ \\
\hline C202 & $0.3455(2)$ & $0.4540(3)$ & $-0.1901(3)$ & $0.0620(17)$ \\
\hline H202 & 0.3684 & 0.4521 & -0.1611 & $0.074 *$ \\
\hline C203 & 0.3240 & $0.3927(4)$ & $-0.2092(4)$ & 0.079 (2) \\
\hline H203 & 0.3326 & 0.3494 & -0.1932 & $0.095 *$ \\
\hline C204 & 0.2907 & $0.3951(4)$ & -0.2508 & $0.076(2)$ \\
\hline H204 & 0.2764 & 0.3535 & -0.2637 & $0.091 *$ \\
\hline $\mathrm{C} 205$ & $0.2776(3)$ & $0.4592(5)$ & $-0.2743(3)$ & $0.081(2)$ \\
\hline H205 & 0.2543 & 0.4607 & -0.3027 & $0.097^{*}$ \\
\hline C206 & $0.2988(2)$ & $0.5206(4)$ & -0.2560 & $0.0653(17)$ \\
\hline H206 & 0.2900 & 0.5638 & -0.2720 & $0.078 *$ \\
\hline C207 & $0.3977(2)$ & $0.6277(3)$ & $-0.2470(2)$ & 0.0485 (13) \\
\hline C208 & $0.4304(3)$ & $0.5804(4)$ & $-0.2676(3)$ & $0.0675(18)$ \\
\hline H208 & 0.4314 & 0.5345 & -0.2531 & $0.081^{*}$ \\
\hline C209 & 0.4618 & $0.6005(5)$ & $-0.3096(3)$ & $0.084(2)$ \\
\hline H209 & 0.4831 & 0.5674 & -0.3245 & $0.101^{*}$ \\
\hline $\mathrm{C} 210$ & $0.4627(3)$ & $0.6665(5)$ & -0.3295 & $0.080(2)$ \\
\hline $\mathrm{H} 210$ & 0.4855 & 0.6798 & -0.3565 & $0.096^{*}$ \\
\hline $\mathrm{C} 211$ & $0.4296(4)$ & $0.7149(5)$ & $-0.3102(3)$ & $0.090(3)$ \\
\hline H211 & 0.4295 & 0.7608 & -0.3246 & $0.108 *$ \\
\hline C212 & $0.3961(3)$ & $0.6950(4)$ & -0.2688 & $0.071(2)$ \\
\hline H212 & 0.3730 & 0.7269 & -0.2561 & $0.085^{*}$ \\
\hline C301 & $0.3552(2)$ & $0.7980(3)$ & $0.0390(3)$ & $0.0555(15)$ \\
\hline C302 & $0.3843(3)$ & 0.8405 (4) & 0.0069 (4) & $0.073(2)$ \\
\hline H302 & 0.4062 & 0.8204 & -0.0185 & $0.087^{*}$ \\
\hline C303 & $0.3814(4)$ & $0.9136(5)$ & $0.0121(5)$ & $0.108(3)$ \\
\hline H303 & 0.4009 & 0.9430 & -0.0098 & $0.130^{*}$ \\
\hline C304 & $0.3495(5)$ & $0.9411(5)$ & $0.0500(6)$ & $0.119(4)$ \\
\hline H304 & 0.3472 & 0.9900 & 0.0536 & $0.143 *$ \\
\hline C305 & $0.3215(4)$ & $0.9003(5)$ & $0.0819(4)$ & $0.099(3)$ \\
\hline H305 & 0.3004 & 0.9211 & 0.1078 & $0.119 *$ \\
\hline C306 & $0.3230(3)$ & $0.8279(4)$ & $0.0774(3)$ & $0.072(2)$ \\
\hline H306 & 0.3030 & 0.7995 & 0.0995 & $0.087^{*}$ \\
\hline C307 & $0.3913(2)$ & $0.6819(4)$ & $0.0979(2)$ & $0.0531(15)$ \\
\hline C308 & 0.4215 & $0.7297(5)$ & 0.1240 & $0.076(2)$ \\
\hline Н308 & 0.4272 & 0.7732 & 0.1068 & $0.092 *$ \\
\hline
\end{tabular}




\begin{tabular}{|c|c|c|c|c|c|}
\hline C309 & $0.4432(3)$ & $0.7140(6)$ & 0.1749 (4) & $0.090(3)$ & \\
\hline H309 & 0.4627 & 0.7472 & 0.1928 & $0.108^{*}$ & \\
\hline $\mathrm{C} 310$ & $0.4358(3)$ & $0.6490(6)$ & $0.1991(3)$ & 0.097 (3) & \\
\hline H310 & 0.4511 & 0.6376 & 0.2333 & $0.116^{*}$ & \\
\hline C311 & $0.4066(4)$ & $0.6015(6)$ & $0.1741(4)$ & 0.107 (3) & \\
\hline H311 & 0.4013 & 0.5577 & 0.1912 & $0.129^{*}$ & \\
\hline $\mathrm{C} 312$ & $0.3845(3)$ & $0.6181(5)$ & 0.1232 & $0.086(2)$ & \\
\hline H312 & 0.3646 & 0.5849 & 0.1058 & $0.103^{*}$ & \\
\hline $\mathrm{C} 401$ & $0.2564(2)$ & $0.5722(3)$ & $-0.0459(2)$ & 0.0507 (14) & \\
\hline $\mathrm{C} 402$ & $0.2583(2)$ & $0.5251(4)$ & -0.0908 & $0.0604(16)$ & \\
\hline $\mathrm{H} 402$ & 0.2879 & 0.5079 & -0.1032 & $0.072 *$ & \\
\hline $\mathrm{C} 403$ & $0.2169(3)$ & $0.5034(4)$ & $-0.1173(3)$ & $0.077(2)$ & \\
\hline $\mathrm{H} 403$ & 0.2183 & 0.4712 & -0.1474 & $0.093 *$ & \\
\hline $\mathrm{C} 404$ & $0.1747(3)$ & $0.5286(5)$ & -0.1000 & $0.091(3)$ & \\
\hline H404 & 0.1468 & 0.5146 & -0.1189 & $0.109^{*}$ & \\
\hline $\mathrm{C} 405$ & $0.1716(3)$ & $0.5739(5)$ & -0.0558 & $0.091(3)$ & \\
\hline $\mathrm{H} 405$ & 0.1417 & 0.5903 & -0.0440 & $0.109^{*}$ & \\
\hline $\mathrm{C} 406$ & $0.2126(2)$ & $0.5962(4)$ & $-0.0277(3)$ & $0.074(2)$ & \\
\hline $\mathrm{H} 406$ & 0.2105 & 0.6271 & 0.0032 & $0.089^{*}$ & \\
\hline $\mathrm{C} 407$ & $0.3282(2)$ & $0.5166(3)$ & $0.0241(2)$ & 0.0494 (14) & \\
\hline $\mathrm{C} 408$ & $0.3714(2)$ & $0.4846(3)$ & $0.0137(3)$ & $0.0570(16)$ & \\
\hline $\mathrm{H} 408$ & 0.3917 & 0.5024 & -0.0145 & $0.068^{*}$ & \\
\hline C409 & $0.3848(3)$ & 0.4258 & 0.0449 & $0.071(2)$ & \\
\hline H409 & 0.4143 & 0.4040 & 0.0383 & $0.085^{*}$ & \\
\hline $\mathrm{C} 410$ & $0.3547(3)$ & $0.4002(4)$ & 0.0855 & $0.076(2)$ & \\
\hline $\mathrm{H} 410$ & 0.3642 & 0.3616 & 0.1073 & $0.091^{*}$ & \\
\hline C411 & $0.3110(3)$ & $0.4297(4)$ & 0.0948 & $0.075(2)$ & \\
\hline H411 & 0.2904 & 0.4104 & 0.1219 & $0.089^{*}$ & \\
\hline C412 & $0.2976(3)$ & 0.4877 (4) & $0.0645(3)$ & $0.0638(18)$ & \\
\hline H412 & 0.2677 & 0.5080 & 0.0708 & $0.077^{*}$ & \\
\hline $\mathrm{C} 12$ & $0.6027(14)$ & $0.889(2)$ & -0.113 & $0.150(19)^{*}$ & 0.25 \\
\hline $\mathrm{C} 13$ & $0.5711(18)$ & $0.867(2)$ & $-0.069(2)$ & $0.18(3)^{*}$ & 0.25 \\
\hline $\mathrm{H} 13$ & 0.5786 & 0.8275 & -0.0469 & $0.216^{*}$ & 0.25 \\
\hline $\mathrm{C} 14$ & $0.5296(18)$ & $0.903(3)$ & $-0.059(2)$ & $0.19(3)^{*}$ & 0.25 \\
\hline H14 & 0.5080 & 0.8870 & -0.0314 & $0.227 *$ & 0.25 \\
\hline $\mathrm{C} 15$ & $0.5195(13)$ & $0.964(2)$ & $-0.0888(16)$ & $0.112(13)^{*}$ & 0.25 \\
\hline H15 & 0.4965 & 0.9958 & -0.0758 & $0.134^{*}$ & 0.25 \\
\hline $\mathrm{C} 16$ & $0.5445(13)$ & $0.9764(19)$ & $-0.1389(15)$ & $0.098(11)^{*}$ & 0.25 \\
\hline H16 & 0.5334 & 1.0100 & -0.1648 & $0.118^{*}$ & 0.25 \\
\hline $\mathrm{C} 17$ & $0.585(2)$ & $0.940(4)$ & $-0.150(2)$ & $0.27(5)^{*}$ & 0.25 \\
\hline H17 & 0.6018 & 0.9488 & -0.1839 & $0.330 *$ & 0.25 \\
\hline $\mathrm{C} 18$ & $0.648(2)$ & $0.844(4)$ & $-0.127(4)$ & $0.22(5)^{*}$ & 0.25 \\
\hline H18A & 0.6544 & 0.8116 & -0.0963 & $0.325 *$ & 0.25 \\
\hline H18B & 0.6424 & 0.8172 & -0.1616 & $0.325^{*}$ & 0.25 \\
\hline $\mathrm{H} 18 \mathrm{C}$ & 0.6751 & 0.8743 & -0.1325 & $0.325^{*}$ & 0.25 \\
\hline C19 & $0.5276(16)$ & $0.991(2)$ & $-0.1188(19)$ & $0.141(18)^{*}$ & 0.25 \\
\hline $\mathrm{C} 20$ & $0.491(2)$ & 1.019 (3) & -0.0841 (19) & $0.22(3)^{*}$ & 0.25 \\
\hline $\mathrm{H} 20$ & 0.4846 & 0.9993 & -0.0487 & $0.262 *$ & 0.25 \\
\hline
\end{tabular}




$\begin{array}{llllll}\text { C21 } & 0.4658(11) & 1.0766(17) & -0.1020(14) & 0.092(10)^{*} & 0.25 \\ \text { H21 } & 0.4423 & 1.0959 & -0.0784 & 0.111^{*} & 0.25 \\ \text { C22 } & 0.4743(15) & 1.106(2) & -0.1540(17) & 0.149(18)^{*} & 0.25 \\ \text { H22 } & 0.4534 & 1.1396 & -0.1687 & 0.178^{*} & 0.25 \\ \text { C23 } & 0.5139(16) & 1.085(2) & -0.1849(15) & 0.152(19)^{*} & 0.25 \\ \text { H23 } & 0.5211 & 1.1076 & -0.2194 & 0.183^{*} & 0.25 \\ \text { C24 } & 0.5423(15) & 1.033(3) & -0.165(2) & 0.18(2)^{*} & 0.25 \\ \text { H24 } & 0.5720 & 1.0251 & -0.1820 & 0.215^{*} & 0.25 \\ \text { C25 } & 0.555(2) & 0.922(2) & -0.103(3) & 0.18(2)^{*} & 0.25 \\ \text { H25A } & 0.5322 & 0.8853 & -0.0952 & 0.269^{*} & 0.25 \\ \text { H25B } & 0.5740 & 0.9307 & -0.0694 & 0.269^{*} & 0.25 \\ \text { H25C } & 0.5753 & 0.9087 & -0.1340 & 0.269^{*} & 0.25 \\ \text { C10 } & 0.6644(7) & 0.7998(10) & -0.1009(12) & 0.182(11) & 0.75 \\ \text { H10A } & 0.6573 & 0.7705 & -0.1339 & 0.218^{*} & 0.75 \\ \text { H10B } & 0.6925 & 0.7800 & -0.0824 & 0.218^{*} & 0.75 \\ \text { C13 } & 0.6794(3) & 0.8884(3) & -0.1262(2) & 0.194(3) & 0.75 \\ \text { C14 } & 0.6209(3) & 0.7945(4) & -0.0581(2) & 0.180(2) & 0.75 \\ \text { C11 } & 0.3470(6) & 1.1480(6) & -0.2714(6) & 0.105(4) & 0.75 \\ \text { H11A } & 0.3661 & 1.1584 & -0.3050 & 0.126^{*} & 0.75 \\ \text { H11B } & 0.3161 & 1.1707 & -0.2764 & 0.126^{*} & 0.75 \\ \text { C15 } & 0.3737(2) & 1.1831(3) & -0.2153(2) & 0.178(2) & 0.75 \\ \text { C16 } & 0.3386(3) & 1.0614(2) & -0.2681(2) & 0.180(3) & 0.75\end{array}$

Atomic displacement parameters $\left(\AA^{2}\right)$

\begin{tabular}{lllllll}
\hline & $U^{11}$ & $U^{22}$ & $U^{33}$ & $U^{12}$ & $U^{13}$ & $U^{23}$ \\
\hline Ir1 & $0.04080(14)$ & $0.03873(13)$ & $0.02908(13)$ & $0.00223(8)$ & $0.00040(8)$ & $0.00056(8)$ \\
P1 & $0.0501(8)$ & $0.0421(7)$ & $0.0342(7)$ & $0.0083(6)$ & $-0.0006(6)$ & $0.0002(6)$ \\
P2 & $0.0471(8)$ & $0.0430(7)$ & $0.0328(7)$ & $0.0031(6)$ & $0.0002(6)$ & $-0.0040(6)$ \\
P3 & $0.0513(8)$ & $0.0505(8)$ & $0.0336(7)$ & $0.0017(7)$ & $0.0012(6)$ & $-0.0051(6)$ \\
P4 & $0.0449(8)$ & $0.0498(8)$ & $0.0365(7)$ & $-0.0025(6)$ & $0.0018(6)$ & $0.0023(6)$ \\
C11 & $0.0656(15)$ & $0.0489(19)$ & $0.0225(18)$ & $0.0087(14)$ & $-0.0159(15)$ & $0.0030(12)$ \\
C11A & $0.080(4)$ & $0.053(4)$ & $0.010(3)$ & $0.041(3)$ & $-0.025(3)$ & $-0.008(2)$ \\
C12 & $0.0687(12)$ & $0.1216(18)$ & $0.0771(12)$ & $-0.0142(11)$ & $-0.0299(10)$ & $-0.0132(12)$ \\
N1 & $0.041(2)$ & $0.045(3)$ & $0.034(2)$ & $0.000(2)$ & $0.0021(19)$ & $0.0033(19)$ \\
N2 & $0.047(3)$ & $0.054(3)$ & $0.040(3)$ & $0.008(2)$ & $0.002(2)$ & $0.001(2)$ \\
N3 & $0.047(3)$ & $0.049(3)$ & $0.041(3)$ & $0.004(2)$ & $-0.001(2)$ & $-0.001(2)$ \\
C1 & $0.048(3)$ & $0.038(3)$ & $0.035(3)$ & $0.002(2)$ & $0.002(2)$ & $0.000(2)$ \\
C2 & $0.046(3)$ & $0.049(3)$ & $0.034(3)$ & $0.005(2)$ & $-0.003(2)$ & $0.000(2)$ \\
C3 & $0.055(4)$ & $0.062(4)$ & $0.040(3)$ & $0.001(3)$ & $0.005(3)$ & $-0.003(3)$ \\
C4 & $0.038(3)$ & $0.049(3)$ & $0.039(3)$ & $-0.002(2)$ & $0.002(2)$ & $0.002(2)$ \\
C5 & $0.060(4)$ & $0.057(4)$ & $0.056(4)$ & $-0.004(3)$ & $-0.004(3)$ & $0.007(3)$ \\
C6 & $0.064(4)$ & $0.062(4)$ & $0.070(4)$ & $-0.022(3)$ & $-0.011(3)$ & $0.000(3)$ \\
C7 & $0.042(3)$ & $0.087(5)$ & $0.053(4)$ & $-0.002(3)$ & $-0.008(3)$ & $-0.013(4)$ \\
C8 & $0.059(4)$ & $0.064(4)$ & $0.050(3)$ & $0.006(3)$ & $-0.013(3)$ & $0.002(3)$ \\
C9 & $0.065(4)$ & $0.050(3)$ & $0.046(3)$ & $-0.003(3)$ & $-0.009(3)$ & $0.003(3)$ \\
C101 & $0.058(4)$ & $0.054(3)$ & $0.040(3)$ & $0.019(3)$ & $0.001(3)$ & $0.001(3)$ \\
C102 & $0.080(5)$ & $0.070(5)$ & $0.054(4)$ & $0.027(4)$ & $-0.010(3)$ & $-0.013(3)$ \\
& & & & &
\end{tabular}




\begin{tabular}{|c|c|c|c|c|c|c|}
\hline $\mathrm{C} 103$ & $0.104(7)$ & $0.088(6)$ & $0.060(4)$ & $0.049(5)$ & -0.001 & $-0.020(4)$ \\
\hline C104 & $0.084(6)$ & $0.110(7)$ & $0.089(6)$ & $0.049(6)$ & $0.015(5)$ & $0.007(5)$ \\
\hline $\mathrm{C} 105$ & $0.064(5)$ & $0.091(6)$ & $0.115(7)$ & $0.021(4)$ & $0.006(5)$ & $-0.011(5)$ \\
\hline C106 & $0.057(4)$ & $0.075(5)$ & $0.072(4)$ & $0.016(3)$ & $-0.001(3)$ & -0.009 (4) \\
\hline C107 & $0.070(4)$ & $0.045(3)$ & $0.043(3)$ & $0.003(3)$ & $-0.002(3)$ & $0.003(3)$ \\
\hline C108 & $0.081(5)$ & $0.070(5)$ & $0.069(5)$ & $-0.010(4)$ & $0.000(4)$ & $0.023(4)$ \\
\hline C109 & $0.100(7)$ & $0.107(7)$ & $0.114(8)$ & $-0.035(6)$ & $0.003(6)$ & $0.034(6)$ \\
\hline $\mathrm{C} 110$ & $0.146(10)$ & $0.079(6)$ & $0.115(8)$ & $-0.025(6)$ & $0.024(7)$ & $0.044(6)$ \\
\hline C111 & $0.160(10)$ & $0.060(5)$ & $0.073(5)$ & $0.008(6)$ & $0.021(6)$ & $0.028(4)$ \\
\hline C112 & $0.098(6)$ & $0.053(4)$ & $0.058(4)$ & $0.013(4)$ & $0.002(4)$ & 0.005 \\
\hline C201 & $0.052(3)$ & $0.051(3)$ & $0.042(3)$ & $-0.001(3)$ & $0.003(3)$ & $-0.003(3)$ \\
\hline $\mathrm{C} 202$ & $0.066(4)$ & $0.053(4)$ & 0.067 (4) & $0.003(3)$ & $-0.009(3)$ & $-0.007(3)$ \\
\hline $\mathrm{C} 203$ & $0.090(6)$ & $0.054(4)$ & $0.093(6)$ & $-0.005(4)$ & $-0.008(5)$ & $-0.012(4)$ \\
\hline C204 & $0.078(5)$ & $0.067(5)$ & $0.082(5)$ & -0.017 (4) & -0.004 & -0.015 (4) \\
\hline $\mathrm{C} 205$ & $0.076(5)$ & $0.094(6)$ & $0.072(5)$ & $-0.017(4)$ & -0.021 & $-0.013(4)$ \\
\hline $\mathrm{C} 206$ & $0.070(4)$ & $0.068(4)$ & $0.057(4)$ & $-0.009(3)$ & -0.013 & $-0.004(3)$ \\
\hline C207 & $0.055(3)$ & $0.059(3)$ & $0.032(3)$ & $0.000(3)$ & -0.001 & $-0.009(3)$ \\
\hline C208 & $0.076(5)$ & $0.070(4)$ & $0.056(4)$ & $0.013(4)$ & $0.016(3)$ & 0.001 \\
\hline C209 & $0.072(5)$ & $0.122(7)$ & $0.058(4)$ & $0.022(5)$ & $0.026(4)$ & $0.009(5)$ \\
\hline $\mathrm{C} 210$ & $0.069(5)$ & $0.113(7)$ & $0.058(4)$ & $-0.013(5)$ & 0.019 (4) & $-0.004(5)$ \\
\hline $\mathrm{C} 211$ & $0.127(8)$ & $0.082(5)$ & $0.061(5)$ & $-0.026(5)$ & $0.021(5)$ & $0.012(4)$ \\
\hline $\mathrm{C} 212$ & $0.094(5)$ & $0.062(4)$ & $0.057(4)$ & $-0.003(4)$ & $0.026(4)$ & $-0.004(3)$ \\
\hline C301 & $0.061(4)$ & $0.057(4)$ & $0.048(3)$ & $0.005(3)$ & $-0.004(3)$ & -0.010 \\
\hline $\mathrm{C} 302$ & $0.086(5)$ & $0.057(4)$ & $0.075(5)$ & $0.001(4)$ & $-0.003(4)$ & $-0.003(4)$ \\
\hline C303 & $0.136(9)$ & $0.051(5)$ & $0.138(9)$ & $-0.010(5)$ & $-0.013(7)$ & $-0.002(6)$ \\
\hline C304 & $0.158(11)$ & $0.053(5)$ & $0.146(10)$ & $0.027(7)$ & $-0.038(9)$ & $-0.025(6)$ \\
\hline C305 & $0.120(8)$ & $0.083(6)$ & $0.096(7)$ & $0.039(6)$ & $-0.016(6)$ & $-0.039(6)$ \\
\hline C306 & $0.079(5)$ & $0.074(5)$ & $0.064(4)$ & $0.021(4)$ & -0.009 (4) & $-0.022(4)$ \\
\hline C307 & $0.056(3)$ & $0.070(4)$ & $0.034(3)$ & $0.003(3)$ & -0.001 & -0.003 \\
\hline C308 & $0.066(4)$ & $0.096(6)$ & $0.067(5)$ & $0.000(4)$ & $-0.012(4)$ & $-0.003(4)$ \\
\hline C309 & $0.067(5)$ & $0.133(8)$ & $0.070(5)$ & $-0.004(5)$ & -0.023 & $-0.021(5)$ \\
\hline C310 & $0.084(6)$ & $0.164(10)$ & $0.042(4)$ & $0.009(6)$ & $-0.016(4)$ & $0.007(5)$ \\
\hline C311 & $0.130(8)$ & $0.131(8)$ & $0.060(5)$ & $-0.017(7)$ & $-0.026(5)$ & $0.038(5)$ \\
\hline C312 & $0.111(7)$ & $0.095(6)$ & $0.051(4)$ & $-0.020(5)$ & -0.022 & $0.019(4)$ \\
\hline $\mathrm{C} 401$ & $0.047(3)$ & $0.056(4)$ & $0.049(3)$ & $-0.007(3)$ & -0.003 & $0.007(3)$ \\
\hline $\mathrm{C} 402$ & $0.061(4)$ & $0.064(4)$ & $0.056(4)$ & $-0.012(3)$ & $0.000(3)$ & $-0.006(3)$ \\
\hline $\mathrm{C} 403$ & $0.073(5)$ & 0.089 (5) & $0.070(5)$ & $-0.020(4)$ & $-0.011(4)$ & $-0.011(4)$ \\
\hline $\mathrm{C} 404$ & $0.074(6)$ & $0.122(7)$ & $0.076(5)$ & $-0.024(5)$ & $-0.021(4)$ & $0.002(5)$ \\
\hline $\mathrm{C} 405$ & $0.054(5)$ & $0.121(8)$ & $0.098(7)$ & $-0.005(5)$ & -0.003 & $-0.003(6)$ \\
\hline C406 & $0.052(4)$ & $0.097(6)$ & $0.074(5)$ & $0.003(4)$ & $-0.004(3)$ & -0.007 (4) \\
\hline $\mathrm{C} 407$ & $0.059(4)$ & $0.050(3)$ & $0.039(3)$ & $-0.012(3)$ & $-0.005(3)$ & $0.003(3)$ \\
\hline $\mathrm{C} 408$ & $0.057(4)$ & $0.055(4)$ & $0.059(4)$ & $-0.007(3)$ & $-0.001(3)$ & $0.011(3)$ \\
\hline C409 & $0.079(5)$ & $0.048(4)$ & $0.085(5)$ & $0.002(3)$ & -0.008 & $0.016(4)$ \\
\hline $\mathrm{C} 410$ & $0.109(6)$ & $0.048(4)$ & $0.070(5)$ & $-0.011(4)$ & $-0.021(5)$ & $0.017(4)$ \\
\hline C411 & $0.099(6)$ & $0.075(5)$ & $0.049(4)$ & $-0.025(4)$ & $0.000(4)$ & $0.021(4)$ \\
\hline $\mathrm{C} 412$ & $0.068(4)$ & $0.071(4)$ & $0.052(4)$ & $-0.012(4)$ & $0.004(3)$ & $0.006(3)$ \\
\hline $\mathrm{C} 10$ & $0.143(15)$ & $0.123(15)$ & $0.28(3)$ & $-0.022(13)$ & 0.029 (19) & $-0.125(18)$ \\
\hline $\mathrm{Cl} 3$ & $0.282(8)$ & $0.178(5)$ & $0.121(4)$ & $-0.029(6)$ & $0.024(4)$ & $-0.015(4)$ \\
\hline
\end{tabular}




\begin{tabular}{lllllll} 
C14 & $0.224(7)$ & $0.181(6)$ & $0.135(4)$ & $-0.024(5)$ & $0.009(4)$ & $-0.033(4)$ \\
C11 & $0.120(11)$ & $0.088(9)$ & $0.106(10)$ & $0.001(7)$ & $-0.036(9)$ & $-0.005(7)$ \\
C15 & $0.249(7)$ & $0.173(5)$ & $0.112(3)$ & $-0.071(4)$ & $-0.039(3)$ & $-0.028(3)$ \\
C16 & $0.315(8)$ & $0.076(2)$ & $0.150(4)$ & $-0.040(3)$ & $-0.071(4)$ & $0.026(2)$ \\
\hline
\end{tabular}

Geometric parameters $\left(\AA,{ }^{\circ}\right)$

\begin{tabular}{|c|c|c|c|}
\hline $\mathrm{Ir} 1-\mathrm{P} 1$ & $2.2788(14)$ & C301-C302 & $1.380(10)$ \\
\hline $\mathrm{Ir} 1-\mathrm{C} 1$ & $1.998(5)$ & $\mathrm{C} 301-\mathrm{C} 306$ & $1.402(10)$ \\
\hline $\operatorname{Ir} 1-\mathrm{N} 1$ & $1.999(4)$ & C302-C303 & $1.405(11)$ \\
\hline Ir1-P3 & $2.3748(14)$ & C302-H302 & 0.9400 \\
\hline Ir1-P4 & $2.2662(14)$ & C303-C304 & $1.372(16)$ \\
\hline $\mathrm{P} 1-\mathrm{C} 107$ & $1.821(6)$ & C $303-\mathrm{H} 303$ & 0.9400 \\
\hline $\mathrm{P} 1-\mathrm{C} 101$ & $1.827(6)$ & C304-C305 & $1.341(15)$ \\
\hline $\mathrm{P} 1-\mathrm{C} 2$ & $1.846(5)$ & C304-H304 & 0.9400 \\
\hline $\mathrm{P} 2-\mathrm{C} 1$ & $1.775(5)$ & $\mathrm{C} 305-\mathrm{C} 306$ & $1.387(12)$ \\
\hline $\mathrm{P} 2-\mathrm{C} 207$ & $1.794(6)$ & C305-H305 & 0.9400 \\
\hline $\mathrm{P} 2-\mathrm{C} 2$ & $1.795(5)$ & С $306-\mathrm{H} 306$ & 0.9400 \\
\hline $\mathrm{P} 2-\mathrm{C} 201$ & $1.803(6)$ & C307-C312 & $1.372(11)$ \\
\hline P3-C301 & $1.802(7)$ & C307-C308 & $1.391(10)$ \\
\hline $\mathrm{P} 3-\mathrm{C} 307$ & $1.828(6)$ & C308-C309 & $1.381(11)$ \\
\hline $\mathrm{P} 3-\mathrm{C} 3$ & $1.851(6)$ & С $308-\mathrm{H} 308$ & 0.9400 \\
\hline P3-P4 & $2.686(2)$ & $\mathrm{C} 309-\mathrm{C} 310$ & $1.384(13)$ \\
\hline $\mathrm{P} 4-\mathrm{C} 401$ & $1.817(6)$ & С $309-\mathrm{H} 309$ & 0.9400 \\
\hline $\mathrm{P} 4-\mathrm{C} 407$ & $1.818(6)$ & $\mathrm{C} 310-\mathrm{C} 311$ & $1.359(13)$ \\
\hline $\mathrm{P} 4-\mathrm{C} 3$ & $1.838(6)$ & C $310-\mathrm{H} 310$ & 0.9400 \\
\hline $\mathrm{C} 11-\mathrm{C} 11 \mathrm{~A}$ & $0.574(6)$ & $\mathrm{C} 311-\mathrm{C} 312$ & $1.392(11)$ \\
\hline $\mathrm{Cl} 2-\mathrm{C} 7$ & $1.751(6)$ & C311-H311 & 0.9400 \\
\hline $\mathrm{N} 1-\mathrm{N} 2$ & $1.354(6)$ & $\mathrm{C} 312-\mathrm{H} 312$ & 0.9400 \\
\hline $\mathrm{N} 1-\mathrm{C} 4$ & $1.431(7)$ & $\mathrm{C} 401-\mathrm{C} 406$ & $1.382(9)$ \\
\hline $\mathrm{N} 2-\mathrm{N} 3$ & $1.308(6)$ & $\mathrm{C} 401-\mathrm{C} 402$ & $1.392(9)$ \\
\hline $\mathrm{N} 3-\mathrm{C} 1$ & $1.346(7)$ & $\mathrm{C} 402-\mathrm{C} 403$ & $1.387(9)$ \\
\hline $\mathrm{C} 2-\mathrm{H} 2 \mathrm{~A}$ & 0.9800 & $\mathrm{C} 402-\mathrm{H} 402$ & 0.9400 \\
\hline $\mathrm{C} 2-\mathrm{H} 2 \mathrm{~B}$ & 0.9800 & $\mathrm{C} 403-\mathrm{C} 404$ & $1.345(12)$ \\
\hline $\mathrm{C} 3-\mathrm{H} 3 \mathrm{~A}$ & 0.9800 & $\mathrm{C} 403-\mathrm{H} 403$ & 0.9400 \\
\hline $\mathrm{C} 3-\mathrm{H} 3 \mathrm{~B}$ & 0.9800 & $\mathrm{C} 404-\mathrm{C} 405$ & $1.361(12)$ \\
\hline $\mathrm{C} 4-\mathrm{C} 5$ & $1.367(8)$ & $\mathrm{C} 404-\mathrm{H} 404$ & 0.9400 \\
\hline $\mathrm{C} 4-\mathrm{C} 9$ & $1.384(8)$ & $\mathrm{C} 405-\mathrm{C} 406$ & $1.398(11)$ \\
\hline $\mathrm{C} 5-\mathrm{C} 6$ & $1.383(9)$ & $\mathrm{C} 405-\mathrm{H} 405$ & 0.9400 \\
\hline $\mathrm{C} 5-\mathrm{H} 5$ & 0.9400 & $\mathrm{C} 406-\mathrm{H} 406$ & 0.9400 \\
\hline $\mathrm{C} 6-\mathrm{C} 7$ & $1.387(10)$ & $\mathrm{C} 407-\mathrm{C} 408$ & $1.383(9)$ \\
\hline $\mathrm{C} 6-\mathrm{H} 6$ & 0.9400 & $\mathrm{C} 407-\mathrm{C} 412$ & $1.398(9)$ \\
\hline $\mathrm{C} 7-\mathrm{C} 8$ & $1.350(9)$ & $\mathrm{C} 408-\mathrm{C} 409$ & $1.396(9)$ \\
\hline $\mathrm{C} 8-\mathrm{C} 9$ & $1.384(8)$ & $\mathrm{C} 408-\mathrm{H} 408$ & 0.9400 \\
\hline $\mathrm{C} 8-\mathrm{H} 8$ & 0.9400 & $\mathrm{C} 409-\mathrm{C} 410$ & $1.369(11)$ \\
\hline C9- $\mathrm{H} 9$ & 0.9400 & $\mathrm{C} 409-\mathrm{H} 409$ & 0.9400 \\
\hline $\mathrm{C} 101-\mathrm{C} 106$ & $1.384(9)$ & $\mathrm{C} 410-\mathrm{C} 411$ & $1.369(11)$ \\
\hline $\mathrm{C} 101-\mathrm{C} 102$ & $1.400(9)$ & $\mathrm{C} 410-\mathrm{H} 410$ & 0.9400 \\
\hline
\end{tabular}




\begin{tabular}{|c|c|c|c|}
\hline $\mathrm{C} 102-\mathrm{C} 103$ & $1.417(11)$ & $\mathrm{C} 411-\mathrm{C} 412$ & $1.371(10)$ \\
\hline C102-H102 & 0.9400 & C411-H411 & 0.9400 \\
\hline $\mathrm{C} 103-\mathrm{C} 104$ & $1.377(13)$ & $\mathrm{C} 412-\mathrm{H} 412$ & 0.9400 \\
\hline C103-H103 & 0.9400 & $\mathrm{C} 12-\mathrm{C} 17$ & $1.401(17)$ \\
\hline $\mathrm{C} 104-\mathrm{C} 105$ & $1.359(13)$ & $\mathrm{C} 12-\mathrm{C} 13$ & $1.430(17)$ \\
\hline C104-H104 & 0.9400 & $\mathrm{C} 12-\mathrm{C} 18$ & $1.575(17)$ \\
\hline $\mathrm{C} 105-\mathrm{C} 106$ & $1.389(10)$ & $\mathrm{C} 13-\mathrm{C} 14$ & $1.381(17)$ \\
\hline $\mathrm{C} 105-\mathrm{H} 105$ & 0.9400 & $\mathrm{C} 13-\mathrm{H} 13$ & 0.9400 \\
\hline C106-H106 & 0.9400 & $\mathrm{C} 14-\mathrm{C} 15$ & $1.388(17)$ \\
\hline $\mathrm{C} 107-\mathrm{C} 108$ & $1.368(10)$ & $\mathrm{C} 14-\mathrm{H} 14$ & 0.9400 \\
\hline $\mathrm{C} 107-\mathrm{C} 112$ & $1.406(9)$ & $\mathrm{C} 15-\mathrm{C} 16$ & $1.397(17)$ \\
\hline $\mathrm{C} 108-\mathrm{C} 109$ & $1.381(11)$ & C15-H15 & 0.9400 \\
\hline $\mathrm{C} 108-\mathrm{H} 108$ & 0.9400 & $\mathrm{C} 16-\mathrm{C} 17$ & $1.369(17)$ \\
\hline $\mathrm{C} 109-\mathrm{C} 110$ & $1.360(14)$ & $\mathrm{C} 16-\mathrm{H} 16$ & 0.9400 \\
\hline C109-H109 & 0.9400 & C17-H17 & 0.9400 \\
\hline $\mathrm{C} 110-\mathrm{C} 111$ & $1.375(14)$ & $\mathrm{C} 18-\mathrm{H} 18 \mathrm{~A}$ & 0.9700 \\
\hline $\mathrm{C} 110-\mathrm{H} 110$ & 0.9400 & $\mathrm{C} 18-\mathrm{H} 18 \mathrm{~B}$ & 0.9700 \\
\hline $\mathrm{C} 111-\mathrm{C} 112$ & $1.356(11)$ & $\mathrm{C} 18-\mathrm{H} 18 \mathrm{C}$ & 0.9700 \\
\hline C111-H111 & 0.9400 & $\mathrm{C} 19-\mathrm{C} 24$ & $1.421(17)$ \\
\hline C112-H112 & 0.9400 & $\mathrm{C} 19-\mathrm{C} 20$ & $1.422(17)$ \\
\hline $\mathrm{C} 201-\mathrm{C} 202$ & $1.382(9)$ & $\mathrm{C} 19-\mathrm{C} 25$ & $1.559(16)$ \\
\hline C201-C206 & $1.395(9)$ & $\mathrm{C} 20-\mathrm{C} 21$ & $1.373(16)$ \\
\hline $\mathrm{C} 202-\mathrm{C} 203$ & $1.394(9)$ & $\mathrm{C} 20-\mathrm{H} 20$ & 0.9400 \\
\hline $\mathrm{C} 202-\mathrm{H} 202$ & 0.9400 & $\mathrm{C} 21-\mathrm{C} 22$ & $1.370(17)$ \\
\hline C203-C204 & $1.361(11)$ & $\mathrm{C} 21-\mathrm{H} 21$ & 0.9400 \\
\hline $\mathrm{C} 203-\mathrm{H} 203$ & 0.9400 & $\mathrm{C} 22-\mathrm{C} 23$ & $1.387(17)$ \\
\hline C204-C205 & $1.392(11)$ & $\mathrm{C} 22-\mathrm{H} 22$ & 0.9400 \\
\hline C204-H204 & 0.9400 & $\mathrm{C} 23-\mathrm{C} 24$ & $1.362(17)$ \\
\hline $\mathrm{C} 205-\mathrm{C} 206$ & $1.385(10)$ & $\mathrm{C} 23-\mathrm{H} 23$ & 0.9400 \\
\hline $\mathrm{C} 205-\mathrm{H} 205$ & 0.9400 & $\mathrm{C} 24-\mathrm{H} 24$ & 0.9400 \\
\hline $\mathrm{C} 206-\mathrm{H} 206$ & 0.9400 & $\mathrm{C} 25-\mathrm{H} 25 \mathrm{~A}$ & 0.9700 \\
\hline C207-C208 & $1.380(9)$ & $\mathrm{C} 25-\mathrm{H} 25 \mathrm{~B}$ & 0.9700 \\
\hline $\mathrm{C} 207-\mathrm{C} 212$ & $1.384(9)$ & $\mathrm{C} 25-\mathrm{H} 25 \mathrm{C}$ & 0.9700 \\
\hline C208-C209 & $1.382(10)$ & $\mathrm{C} 10-\mathrm{Cl} 4$ & $1.59(2)$ \\
\hline $\mathrm{C} 208-\mathrm{H} 208$ & 0.9400 & $\mathrm{C} 10-\mathrm{Cl} 3$ & $1.84(2)$ \\
\hline C209-C210 & $1.347(12)$ & $\mathrm{C} 10-\mathrm{H} 10 \mathrm{~A}$ & 0.9800 \\
\hline С209-H209 & 0.9400 & $\mathrm{C} 10-\mathrm{H} 10 \mathrm{~B}$ & 0.9800 \\
\hline $\mathrm{C} 210-\mathrm{C} 211$ & $1.389(12)$ & $\mathrm{C} 11-\mathrm{Cl} 5$ & $1.665(13)$ \\
\hline $\mathrm{C} 210-\mathrm{H} 210$ & 0.9400 & $\mathrm{C} 11-\mathrm{Cl} 6$ & $1.672(12)$ \\
\hline C211-C212 & $1.411(10)$ & $\mathrm{C} 11-\mathrm{H} 11 \mathrm{~A}$ & 0.9800 \\
\hline $\mathrm{C} 211-\mathrm{H} 211$ & 0.9400 & C11-H11B & 0.9800 \\
\hline $\mathrm{C} 212-\mathrm{H} 212$ & 0.9400 & & \\
\hline $\mathrm{C} 1-\mathrm{Ir} 1-\mathrm{N} 1$ & $73.7(2)$ & $\mathrm{C} 208-\mathrm{C} 209-\mathrm{H} 209$ & 119.2 \\
\hline $\mathrm{C} 1-\mathrm{Ir} 1-\mathrm{P} 4$ & $108.92(16)$ & $\mathrm{C} 209-\mathrm{C} 210-\mathrm{C} 211$ & $119.7(7)$ \\
\hline $\mathrm{N} 1-\mathrm{Ir} 1-\mathrm{P} 4$ & $122.71(13)$ & $\mathrm{C} 209-\mathrm{C} 210-\mathrm{H} 210$ & 120.1 \\
\hline $\mathrm{C} 1-\mathrm{Ir} 1-\mathrm{P} 1$ & $85.35(15)$ & $\mathrm{C} 211-\mathrm{C} 210-\mathrm{H} 210$ & 120.1 \\
\hline $\mathrm{N} 1-\mathrm{Ir} 1-\mathrm{P} 1$ & $140.40(13)$ & $\mathrm{C} 210-\mathrm{C} 211-\mathrm{C} 212$ & $119.7(8)$ \\
\hline
\end{tabular}




\begin{tabular}{|c|c|c|c|}
\hline $\mathrm{P} 4-\mathrm{Ir} 1-\mathrm{P} 1$ & $95.59(5)$ & $\mathrm{C} 210-\mathrm{C} 211-\mathrm{H} 211$ & 120.1 \\
\hline $\mathrm{C} 1-\mathrm{Ir} 1-\mathrm{P} 3$ & $173.04(16)$ & $\mathrm{C} 212-\mathrm{C} 211-\mathrm{H} 211$ & 120.1 \\
\hline $\mathrm{N} 1-\mathrm{Ir} 1-\mathrm{P} 3$ & $100.49(13)$ & $\mathrm{C} 207-\mathrm{C} 212-\mathrm{C} 211$ & $119.1(7)$ \\
\hline $\mathrm{P} 4-\mathrm{Ir} 1-\mathrm{P} 3$ & $70.69(5)$ & $\mathrm{C} 207-\mathrm{C} 212-\mathrm{H} 212$ & 120.4 \\
\hline $\mathrm{P} 1-\mathrm{Ir} 1-\mathrm{P} 3$ & $101.62(5)$ & $\mathrm{C} 211-\mathrm{C} 212-\mathrm{H} 212$ & 120.4 \\
\hline $\mathrm{C} 107-\mathrm{P} 1-\mathrm{C} 101$ & $102.1(3)$ & $\mathrm{C} 302-\mathrm{C} 301-\mathrm{C} 306$ & $120.0(7)$ \\
\hline $\mathrm{C} 107-\mathrm{P} 1-\mathrm{C} 2$ & $102.9(3)$ & $\mathrm{C} 302-\mathrm{C} 301-\mathrm{P} 3$ & $118.8(5)$ \\
\hline $\mathrm{C} 101-\mathrm{P} 1-\mathrm{C} 2$ & $102.3(3)$ & $\mathrm{C} 306-\mathrm{C} 301-\mathrm{P} 3$ & $121.1(6)$ \\
\hline $\mathrm{C} 107-\mathrm{P} 1-\mathrm{Ir} 1$ & $115.4(2)$ & $\mathrm{C} 301-\mathrm{C} 302-\mathrm{C} 303$ & $120.0(9)$ \\
\hline $\mathrm{C} 101-\mathrm{P} 1-\mathrm{Ir} 1$ & $124.54(19)$ & $\mathrm{C} 301-\mathrm{C} 302-\mathrm{H} 302$ & 120.0 \\
\hline $\mathrm{C} 2-\mathrm{P} 1-\mathrm{Ir} 1$ & $106.97(18)$ & $\mathrm{C} 303-\mathrm{C} 302-\mathrm{H} 302$ & 120.0 \\
\hline $\mathrm{C} 1-\mathrm{P} 2-\mathrm{C} 207$ & $111.2(3)$ & $\mathrm{C} 304-\mathrm{C} 303-\mathrm{C} 302$ & $118.5(10)$ \\
\hline $\mathrm{C} 1-\mathrm{P} 2-\mathrm{C} 2$ & $103.4(2)$ & $\mathrm{C} 304-\mathrm{C} 303-\mathrm{H} 303$ & 120.8 \\
\hline $\mathrm{C} 207-\mathrm{P} 2-\mathrm{C} 2$ & $112.2(3)$ & $\mathrm{C} 302-\mathrm{C} 303-\mathrm{H} 303$ & 120.8 \\
\hline $\mathrm{C} 1-\mathrm{P} 2-\mathrm{C} 201$ & $115.6(3)$ & $\mathrm{C} 305-\mathrm{C} 304-\mathrm{C} 303$ & $121.9(9)$ \\
\hline C207-P2-C201 & $106.5(3)$ & $\mathrm{C} 305-\mathrm{C} 304-\mathrm{H} 304$ & 119.0 \\
\hline $\mathrm{C} 2-\mathrm{P} 2-\mathrm{C} 201$ & $108.0(3)$ & $\mathrm{C} 303-\mathrm{C} 304-\mathrm{H} 304$ & 119.0 \\
\hline C301-P3-C307 & $101.4(3)$ & $\mathrm{C} 304-\mathrm{C} 305-\mathrm{C} 306$ & $121.2(9)$ \\
\hline $\mathrm{C} 301-\mathrm{P} 3-\mathrm{C} 3$ & $108.6(3)$ & $\mathrm{C} 304-\mathrm{C} 305-\mathrm{H} 305$ & 119.4 \\
\hline $\mathrm{C} 307-\mathrm{P} 3-\mathrm{C} 3$ & $103.9(3)$ & $\mathrm{C} 306-\mathrm{C} 305-\mathrm{H} 305$ & 119.4 \\
\hline C301-P3-Ir1 & $122.3(2)$ & $\mathrm{C} 305-\mathrm{C} 306-\mathrm{C} 301$ & $118.4(9)$ \\
\hline C307-P3-Ir1 & $124.5(2)$ & C305-C306-H306 & 120.8 \\
\hline $\mathrm{C} 3-\mathrm{P} 3-\mathrm{Ir} 1$ & $93.54(19)$ & $\mathrm{C} 301-\mathrm{C} 306-\mathrm{H} 306$ & 120.8 \\
\hline $\mathrm{C} 301-\mathrm{P} 3-\mathrm{P} 4$ & $138.8(2)$ & C312-C307-C308 & $118.3(6)$ \\
\hline C307-P3-P4 & $113.3(2)$ & C312-C307-P3 & $120.9(5)$ \\
\hline $\mathrm{C} 3-\mathrm{P} 3-\mathrm{P} 4$ & $43.06(19)$ & $\mathrm{C} 308-\mathrm{C} 307-\mathrm{P} 3$ & $120.7(5)$ \\
\hline $\mathrm{Ir} 1-\mathrm{P} 3-\mathrm{P} 4$ & $52.76(4)$ & $\mathrm{C} 309-\mathrm{C} 308-\mathrm{C} 307$ & $120.9(8)$ \\
\hline C401-P4-C407 & $101.7(3)$ & С $309-\mathrm{C} 308-\mathrm{H} 308$ & 119.5 \\
\hline $\mathrm{C} 401-\mathrm{P} 4-\mathrm{C} 3$ & $109.2(3)$ & С $307-\mathrm{C} 308-\mathrm{H} 308$ & 119.5 \\
\hline $\mathrm{C} 407-\mathrm{P} 4-\mathrm{C} 3$ & $107.9(3)$ & $\mathrm{C} 308-\mathrm{C} 309-\mathrm{C} 310$ & $119.3(8)$ \\
\hline $\mathrm{C} 401-\mathrm{P} 4-\mathrm{Ir} 1$ & $126.1(2)$ & C308-C309-H309 & 120.4 \\
\hline $\mathrm{C} 407-\mathrm{P} 4-\mathrm{Ir} 1$ & $113.5(2)$ & С $310-\mathrm{C} 309-\mathrm{H} 309$ & 120.4 \\
\hline C3-P4-Ir1 & $97.6(2)$ & C311-C310-C309 & $120.6(8)$ \\
\hline $\mathrm{C} 401-\mathrm{P} 4-\mathrm{P} 3$ & $143.7(2)$ & $\mathrm{C} 311-\mathrm{C} 310-\mathrm{H} 310$ & 119.7 \\
\hline $\mathrm{C} 407-\mathrm{P} 4-\mathrm{P} 3$ & $109.19(19)$ & $\mathrm{C} 309-\mathrm{C} 310-\mathrm{H} 310$ & 119.7 \\
\hline $\mathrm{C} 3-\mathrm{P} 4-\mathrm{P} 3$ & $43.4(2)$ & $\mathrm{C} 310-\mathrm{C} 311-\mathrm{C} 312$ & $119.6(9)$ \\
\hline $\mathrm{Ir} 1-\mathrm{P} 4-\mathrm{P} 3$ & $56.54(5)$ & $\mathrm{C} 310-\mathrm{C} 311-\mathrm{H} 311$ & 120.2 \\
\hline $\mathrm{N} 2-\mathrm{N} 1-\mathrm{C} 4$ & $111.5(4)$ & $\mathrm{C} 312-\mathrm{C} 311-\mathrm{H} 311$ & 120.2 \\
\hline $\mathrm{N} 2-\mathrm{N} 1-\mathrm{Ir} 1$ & $119.6(3)$ & $\mathrm{C} 307-\mathrm{C} 312-\mathrm{C} 311$ & $121.1(8)$ \\
\hline $\mathrm{C} 4-\mathrm{N} 1-\mathrm{Ir} 1$ & $128.9(4)$ & C307-C $312-\mathrm{H} 312$ & 119.4 \\
\hline $\mathrm{N} 3-\mathrm{N} 2-\mathrm{N} 1$ & $112.3(4)$ & C311-C312-H312 & 119.4 \\
\hline $\mathrm{N} 2-\mathrm{N} 3-\mathrm{C} 1$ & $115.2(4)$ & $\mathrm{C} 406-\mathrm{C} 401-\mathrm{C} 402$ & $119.2(6)$ \\
\hline $\mathrm{N} 3-\mathrm{C} 1-\mathrm{P} 2$ & $115.9(4)$ & $\mathrm{C} 406-\mathrm{C} 401-\mathrm{P} 4$ & $123.4(5)$ \\
\hline $\mathrm{N} 3-\mathrm{C} 1-\mathrm{Ir} 1$ & $118.5(4)$ & $\mathrm{C} 402-\mathrm{C} 401-\mathrm{P} 4$ & $117.4(5)$ \\
\hline $\mathrm{P} 2-\mathrm{C} 1-\mathrm{Ir} 1$ & $124.5(3)$ & $\mathrm{C} 403-\mathrm{C} 402-\mathrm{C} 401$ & $120.3(7)$ \\
\hline $\mathrm{P} 2-\mathrm{C} 2-\mathrm{P} 1$ & $109.6(3)$ & $\mathrm{C} 403-\mathrm{C} 402-\mathrm{H} 402$ & 119.8 \\
\hline $\mathrm{P} 2-\mathrm{C} 2-\mathrm{H} 2 \mathrm{~A}$ & 109.7 & $\mathrm{C} 401-\mathrm{C} 402-\mathrm{H} 402$ & 119.8 \\
\hline $\mathrm{P} 1-\mathrm{C} 2-\mathrm{H} 2 \mathrm{~A}$ & 109.7 & $\mathrm{C} 404-\mathrm{C} 403-\mathrm{C} 402$ & $119.8(8)$ \\
\hline
\end{tabular}




\begin{tabular}{|c|c|}
\hline $\mathrm{P} 2-\mathrm{C} 2-\mathrm{H} 2 \mathrm{~B}$ & 109.7 \\
\hline $\mathrm{P} 1-\mathrm{C} 2-\mathrm{H} 2 \mathrm{~B}$ & 109.7 \\
\hline $\mathrm{H} 2 \mathrm{~A}-\mathrm{C} 2-\mathrm{H} 2 \mathrm{~B}$ & 108.2 \\
\hline $\mathrm{P} 4-\mathrm{C} 3-\mathrm{P} 3$ & $93.5(3)$ \\
\hline $\mathrm{P} 4-\mathrm{C} 3-\mathrm{H} 3 \mathrm{~A}$ & 113.0 \\
\hline $\mathrm{P} 3-\mathrm{C} 3-\mathrm{H} 3 \mathrm{~A}$ & 113.0 \\
\hline $\mathrm{P} 4-\mathrm{C} 3-\mathrm{H} 3 \mathrm{~B}$ & 113.0 \\
\hline $\mathrm{P} 3-\mathrm{C} 3-\mathrm{H} 3 \mathrm{~B}$ & 113.0 \\
\hline $\mathrm{H} 3 \mathrm{~A}-\mathrm{C} 3-\mathrm{H} 3 \mathrm{~B}$ & 110.4 \\
\hline $\mathrm{C} 5-\mathrm{C} 4-\mathrm{C} 9$ & $119.7(5)$ \\
\hline $\mathrm{C} 5-\mathrm{C} 4-\mathrm{N} 1$ & $119.2(5)$ \\
\hline $\mathrm{C} 9-\mathrm{C} 4-\mathrm{N} 1$ & $121.1(5)$ \\
\hline $\mathrm{C} 4-\mathrm{C} 5-\mathrm{C} 6$ & $120.5(6)$ \\
\hline $\mathrm{C} 4-\mathrm{C} 5-\mathrm{H} 5$ & 119.7 \\
\hline $\mathrm{C} 6-\mathrm{C} 5-\mathrm{H} 5$ & 119.7 \\
\hline $\mathrm{C} 5-\mathrm{C} 6-\mathrm{C} 7$ & $118.4(6)$ \\
\hline $\mathrm{C} 5-\mathrm{C} 6-\mathrm{H} 6$ & 120.8 \\
\hline $\mathrm{C} 7-\mathrm{C} 6-\mathrm{H} 6$ & 120.8 \\
\hline $\mathrm{C} 8-\mathrm{C} 7-\mathrm{C} 6$ & $122.0(6)$ \\
\hline $\mathrm{C} 8-\mathrm{C} 7-\mathrm{Cl} 2$ & $119.0(6)$ \\
\hline $\mathrm{C} 6-\mathrm{C} 7-\mathrm{Cl} 2$ & $118.9(6)$ \\
\hline $\mathrm{C} 7-\mathrm{C} 8-\mathrm{C} 9$ & $118.9(6)$ \\
\hline $\mathrm{C} 7-\mathrm{C} 8-\mathrm{H} 8$ & 120.5 \\
\hline $\mathrm{C} 9-\mathrm{C} 8-\mathrm{H} 8$ & 120.5 \\
\hline $\mathrm{C} 4-\mathrm{C} 9-\mathrm{C} 8$ & $120.4(6)$ \\
\hline $\mathrm{C} 4-\mathrm{C} 9-\mathrm{H} 9$ & 119.8 \\
\hline $\mathrm{C} 8-\mathrm{C} 9-\mathrm{H} 9$ & 119.8 \\
\hline $\mathrm{C} 106-\mathrm{C} 101-\mathrm{C} 102$ & $118.6(6)$ \\
\hline $\mathrm{C} 106-\mathrm{C} 101-\mathrm{P} 1$ & $123.3(5)$ \\
\hline $\mathrm{C} 102-\mathrm{C} 101-\mathrm{P} 1$ & $117.9(5)$ \\
\hline $\mathrm{C} 101-\mathrm{C} 102-\mathrm{C} 103$ & $119.2(8)$ \\
\hline $\mathrm{C} 101-\mathrm{C} 102-\mathrm{H} 102$ & 120.4 \\
\hline $\mathrm{C} 103-\mathrm{C} 102-\mathrm{H} 102$ & 120.4 \\
\hline $\mathrm{C} 104-\mathrm{C} 103-\mathrm{C} 102$ & $119.7(7)$ \\
\hline $\mathrm{C} 104-\mathrm{C} 103-\mathrm{H} 103$ & 120.1 \\
\hline $\mathrm{C} 102-\mathrm{C} 103-\mathrm{H} 103$ & 120.1 \\
\hline $\mathrm{C} 105-\mathrm{C} 104-\mathrm{C} 103$ & $121.4(8)$ \\
\hline $\mathrm{C} 105-\mathrm{C} 104-\mathrm{H} 104$ & 119.3 \\
\hline $\mathrm{C} 103-\mathrm{C} 104-\mathrm{H} 104$ & 119.3 \\
\hline $\mathrm{C} 104-\mathrm{C} 105-\mathrm{C} 106$ & $119.2(9)$ \\
\hline $\mathrm{C} 104-\mathrm{C} 105-\mathrm{H} 105$ & 120.4 \\
\hline $\mathrm{C} 106-\mathrm{C} 105-\mathrm{H} 105$ & 120.4 \\
\hline $\mathrm{C} 101-\mathrm{C} 106-\mathrm{C} 105$ & $121.8(7)$ \\
\hline $\mathrm{C} 101-\mathrm{C} 106-\mathrm{H} 106$ & 119.1 \\
\hline $\mathrm{C} 105-\mathrm{C} 106-\mathrm{H} 106$ & 119.1 \\
\hline $\mathrm{C} 108-\mathrm{C} 107-\mathrm{C} 112$ & $118.0(6)$ \\
\hline $\mathrm{C} 108-\mathrm{C} 107-\mathrm{P} 1$ & $120.8(5)$ \\
\hline $\mathrm{C} 112-\mathrm{C} 107-\mathrm{P} 1$ & $121.1(6)$ \\
\hline
\end{tabular}

$\begin{array}{ll}\mathrm{C} 404-\mathrm{C} 403-\mathrm{H} 403 & 120.1 \\ \mathrm{C} 402-\mathrm{C} 403-\mathrm{H} 403 & 120.1 \\ \mathrm{C} 403-\mathrm{C} 404-\mathrm{C} 405 & 121.2(8) \\ \mathrm{C} 403-\mathrm{C} 404-\mathrm{H} 404 & 119.4 \\ \mathrm{C} 405-\mathrm{C} 404-\mathrm{H} 404 & 119.4 \\ \mathrm{C} 404-\mathrm{C} 405-\mathrm{C} 406 & 120.4(8) \\ \mathrm{C} 404-\mathrm{C} 405-\mathrm{H} 405 & 119.8 \\ \mathrm{C} 406-\mathrm{C} 405-\mathrm{H} 405 & 119.8 \\ \mathrm{C} 401-\mathrm{C} 406-\mathrm{C} 405 & 119.0(8) \\ \mathrm{C} 401-\mathrm{C} 406-\mathrm{H} 406 & 120.5 \\ \mathrm{C} 405-\mathrm{C} 406-\mathrm{H} 406 & 120.5 \\ \mathrm{C} 408-\mathrm{C} 407-\mathrm{C} 412 & 119.2(6) \\ \mathrm{C} 408-\mathrm{C} 407-\mathrm{P} 4 & 120.2(4) \\ \mathrm{C} 412-\mathrm{C} 407-\mathrm{P} 4 & 120.6(5) \\ \mathrm{C} 407-\mathrm{C} 408-\mathrm{C} 409 & 119.9(6) \\ \mathrm{C} 407-\mathrm{C} 408-\mathrm{H} 408 & 120.1\end{array}$

$\mathrm{C} 407-\mathrm{C} 408-\mathrm{H} 408 \quad 120.1$

$\mathrm{C} 409-\mathrm{C} 408-\mathrm{H} 408 \quad 120.1$

$\mathrm{C} 410-\mathrm{C} 409-\mathrm{C} 408 \quad 119.4$ (7)

$\mathrm{C} 410-\mathrm{C} 409-\mathrm{H} 409 \quad 120.3$

$\mathrm{C} 408-\mathrm{C} 409-\mathrm{H} 409 \quad 120.3$

$\mathrm{C} 409-\mathrm{C} 410-\mathrm{C} 411$

$\mathrm{C} 409-\mathrm{C} 410-\mathrm{H} 410 \quad 119.3$

$\mathrm{C} 411-\mathrm{C} 410-\mathrm{H} 410 \quad 119.3$

$\mathrm{C} 410-\mathrm{C} 411-\mathrm{C} 412 \quad 119.6$ (7)

$\mathrm{C} 410-\mathrm{C} 411-\mathrm{H} 411 \quad 120.2$

$\mathrm{C} 412-\mathrm{C} 411-\mathrm{H} 411 \quad 120.2$

$\mathrm{C} 411-\mathrm{C} 412-\mathrm{C} 407 \quad 120.4$ (7)

$\mathrm{C} 411-\mathrm{C} 412-\mathrm{H} 412 \quad 119.8$

$\mathrm{C} 407-\mathrm{C} 412-\mathrm{H} 412 \quad 119.8$

$\mathrm{C} 17-\mathrm{C} 12-\mathrm{C} 13 \quad 116.2(15)$

$\mathrm{C} 17-\mathrm{C} 12-\mathrm{C} 18 \quad 122.0(18)$

$\mathrm{C} 13-\mathrm{C} 12-\mathrm{C} 18 \quad 119.9(18)$

$\mathrm{C} 14-\mathrm{C} 13-\mathrm{C} 12 \quad 120.4(15)$

$\mathrm{C} 14-\mathrm{C} 13-\mathrm{H} 13 \quad 119.8$

$\mathrm{C} 12-\mathrm{C} 13-\mathrm{H} 13 \quad 119.8$

$\mathrm{C} 13-\mathrm{C} 14-\mathrm{C} 15 \quad 120.2$ (16)

$\mathrm{C} 13-\mathrm{C} 14-\mathrm{H} 14 \quad 119.9$

$\mathrm{C} 15-\mathrm{C} 14-\mathrm{H} 14 \quad 119.9$

$\mathrm{C} 14-\mathrm{C} 15-\mathrm{C} 16 \quad 118.2$ (16)

$\mathrm{C} 14-\mathrm{C} 15-\mathrm{H} 15 \quad 120.9$

$\mathrm{C} 16-\mathrm{C} 15-\mathrm{H} 15 \quad 120.9$

$\mathrm{C} 17-\mathrm{C} 16-\mathrm{C} 15 \quad 120.0(15)$

$\mathrm{C} 17-\mathrm{C} 16-\mathrm{H} 16 \quad 120.0$

$\mathrm{C} 15-\mathrm{C} 16-\mathrm{H} 16 \quad 120.0$

$\mathrm{C} 16-\mathrm{C} 17-\mathrm{C} 12$

$\mathrm{C} 16-\mathrm{C} 17-\mathrm{H} 17 \quad 119.1$

$\mathrm{C} 12-\mathrm{C} 17-\mathrm{H} 17 \quad 119.1$

$\mathrm{C} 12-\mathrm{C} 18-\mathrm{H} 18 \mathrm{~A} \quad 109.5$ 


\begin{tabular}{|c|c|c|c|}
\hline $\mathrm{C} 107-\mathrm{C} 108-\mathrm{C} 109$ & $120.7(8)$ & $\mathrm{C} 12-\mathrm{C} 18-\mathrm{H} 18 \mathrm{~B}$ & 109.5 \\
\hline C107-C108-H108 & 119.7 & $\mathrm{H} 18 \mathrm{~A}-\mathrm{C} 18-\mathrm{H} 18 \mathrm{~B}$ & 109.5 \\
\hline $\mathrm{C} 109-\mathrm{C} 108-\mathrm{H} 108$ & 119.7 & $\mathrm{C} 12-\mathrm{C} 18-\mathrm{H} 18 \mathrm{C}$ & 109.5 \\
\hline $\mathrm{C} 110-\mathrm{C} 109-\mathrm{C} 108$ & $120.3(10)$ & $\mathrm{H} 18 \mathrm{~A}-\mathrm{C} 18-\mathrm{H} 18 \mathrm{C}$ & 109.5 \\
\hline C110-C109-H109 & 119.8 & $\mathrm{H} 18 \mathrm{~B}-\mathrm{C} 18-\mathrm{H} 18 \mathrm{C}$ & 109.5 \\
\hline C108-C109-H109 & 119.8 & $\mathrm{C} 24-\mathrm{C} 19-\mathrm{C} 20$ & $115.5(14)$ \\
\hline $\mathrm{C} 109-\mathrm{C} 110-\mathrm{C} 111$ & $120.2(8)$ & $\mathrm{C} 24-\mathrm{C} 19-\mathrm{C} 25$ & $121.4(16)$ \\
\hline $\mathrm{C} 109-\mathrm{C} 110-\mathrm{H} 110$ & 119.9 & $\mathrm{C} 20-\mathrm{C} 19-\mathrm{C} 25$ & $122.5(16)$ \\
\hline $\mathrm{C} 111-\mathrm{C} 110-\mathrm{H} 110$ & 119.9 & $\mathrm{C} 21-\mathrm{C} 20-\mathrm{C} 19$ & $120.4(15)$ \\
\hline $\mathrm{C} 112-\mathrm{C} 111-\mathrm{C} 110$ & $119.8(9)$ & $\mathrm{C} 21-\mathrm{C} 20-\mathrm{H} 20$ & 119.8 \\
\hline $\mathrm{C} 112-\mathrm{C} 111-\mathrm{H} 111$ & 120.1 & $\mathrm{C} 19-\mathrm{C} 20-\mathrm{H} 20$ & 119.8 \\
\hline $\mathrm{C} 110-\mathrm{C} 111-\mathrm{H} 111$ & 120.1 & $\mathrm{C} 22-\mathrm{C} 21-\mathrm{C} 20$ & $120.5(15)$ \\
\hline $\mathrm{C} 111-\mathrm{C} 112-\mathrm{C} 107$ & $121.1(9)$ & $\mathrm{C} 22-\mathrm{C} 21-\mathrm{H} 21$ & 119.7 \\
\hline $\mathrm{C} 111-\mathrm{C} 112-\mathrm{H} 112$ & 119.5 & $\mathrm{C} 20-\mathrm{C} 21-\mathrm{H} 21$ & 119.7 \\
\hline $\mathrm{C} 107-\mathrm{C} 112-\mathrm{H} 112$ & 119.5 & $\mathrm{C} 21-\mathrm{C} 22-\mathrm{C} 23$ & $119.9(15)$ \\
\hline $\mathrm{C} 202-\mathrm{C} 201-\mathrm{C} 206$ & $119.9(6)$ & $\mathrm{C} 21-\mathrm{C} 22-\mathrm{H} 22$ & 120.0 \\
\hline $\mathrm{C} 202-\mathrm{C} 201-\mathrm{P} 2$ & $121.9(5)$ & $\mathrm{C} 23-\mathrm{C} 22-\mathrm{H} 22$ & 120.0 \\
\hline $\mathrm{C} 206-\mathrm{C} 201-\mathrm{P} 2$ & $118.3(5)$ & $\mathrm{C} 24-\mathrm{C} 23-\mathrm{C} 22$ & $119.6(15)$ \\
\hline $\mathrm{C} 201-\mathrm{C} 202-\mathrm{C} 203$ & $120.1(7)$ & $\mathrm{C} 24-\mathrm{C} 23-\mathrm{H} 23$ & 120.2 \\
\hline $\mathrm{C} 201-\mathrm{C} 202-\mathrm{H} 202$ & 120.0 & $\mathrm{C} 22-\mathrm{C} 23-\mathrm{H} 23$ & 120.2 \\
\hline $\mathrm{C} 203-\mathrm{C} 202-\mathrm{H} 202$ & 120.0 & $\mathrm{C} 23-\mathrm{C} 24-\mathrm{C} 19$ & $120.9(17)$ \\
\hline $\mathrm{C} 204-\mathrm{C} 203-\mathrm{C} 202$ & $120.3(7)$ & $\mathrm{C} 23-\mathrm{C} 24-\mathrm{H} 24$ & 119.5 \\
\hline $\mathrm{C} 204-\mathrm{C} 203-\mathrm{H} 203$ & 119.8 & $\mathrm{C} 19-\mathrm{C} 24-\mathrm{H} 24$ & 119.5 \\
\hline $\mathrm{C} 202-\mathrm{C} 203-\mathrm{H} 203$ & 119.8 & $\mathrm{C} 19-\mathrm{C} 25-\mathrm{H} 25 \mathrm{~A}$ & 109.5 \\
\hline C203-C204-C205 & $119.9(7)$ & $\mathrm{C} 19-\mathrm{C} 25-\mathrm{H} 25 \mathrm{~B}$ & 109.5 \\
\hline C203-C204-H204 & 120.0 & $\mathrm{H} 25 \mathrm{~A}-\mathrm{C} 25-\mathrm{H} 25 \mathrm{~B}$ & 109.5 \\
\hline C205-C204-H204 & 120.0 & $\mathrm{C} 19-\mathrm{C} 25-\mathrm{H} 25 \mathrm{C}$ & 109.5 \\
\hline $\mathrm{C} 206-\mathrm{C} 205-\mathrm{C} 204$ & $120.5(7)$ & $\mathrm{H} 25 \mathrm{~A}-\mathrm{C} 25-\mathrm{H} 25 \mathrm{C}$ & 109.5 \\
\hline $\mathrm{C} 206-\mathrm{C} 205-\mathrm{H} 205$ & 119.7 & $\mathrm{H} 25 \mathrm{~B}-\mathrm{C} 25-\mathrm{H} 25 \mathrm{C}$ & 109.5 \\
\hline $\mathrm{C} 204-\mathrm{C} 205-\mathrm{H} 205$ & 119.7 & $\mathrm{Cl} 4-\mathrm{C} 10-\mathrm{Cl} 3$ & $116.1(11)$ \\
\hline $\mathrm{C} 205-\mathrm{C} 206-\mathrm{C} 201$ & $119.2(7)$ & $\mathrm{Cl} 4-\mathrm{C} 10-\mathrm{H} 10 \mathrm{~A}$ & 108.3 \\
\hline $\mathrm{C} 205-\mathrm{C} 206-\mathrm{H} 206$ & 120.4 & $\mathrm{Cl} 3-\mathrm{C} 10-\mathrm{H} 10 \mathrm{~A}$ & 108.3 \\
\hline $\mathrm{C} 201-\mathrm{C} 206-\mathrm{H} 206$ & 120.4 & $\mathrm{Cl} 4-\mathrm{C} 10-\mathrm{H} 10 \mathrm{~B}$ & 108.3 \\
\hline $\mathrm{C} 208-\mathrm{C} 207-\mathrm{C} 212$ & $119.9(6)$ & $\mathrm{Cl} 3-\mathrm{C} 10-\mathrm{H} 10 \mathrm{~B}$ & 108.3 \\
\hline $\mathrm{C} 208-\mathrm{C} 207-\mathrm{P} 2$ & $117.1(5)$ & $\mathrm{H} 10 \mathrm{~A}-\mathrm{C} 10-\mathrm{H} 10 \mathrm{~B}$ & 107.4 \\
\hline $\mathrm{C} 212-\mathrm{C} 207-\mathrm{P} 2$ & $123.0(5)$ & $\mathrm{Cl} 5-\mathrm{C} 11-\mathrm{Cl} 6$ & $115.1(8)$ \\
\hline C207-C208-C209 & $119.8(7)$ & $\mathrm{Cl} 5-\mathrm{C} 11-\mathrm{H} 11 \mathrm{~A}$ & 108.5 \\
\hline C207-C208-H208 & 120.1 & $\mathrm{C} 16-\mathrm{C} 11-\mathrm{H} 11 \mathrm{~A}$ & 108.5 \\
\hline C209-C208-H208 & 120.1 & $\mathrm{Cl} 5-\mathrm{C} 11-\mathrm{H} 11 \mathrm{~B}$ & 108.5 \\
\hline $\mathrm{C} 210-\mathrm{C} 209-\mathrm{C} 208$ & $121.6(8)$ & $\mathrm{C} 16-\mathrm{C} 11-\mathrm{H} 11 \mathrm{~B}$ & 108.5 \\
\hline $\mathrm{C} 210-\mathrm{C} 209-\mathrm{H} 209$ & 119.2 & $\mathrm{H} 11 \mathrm{~A}-\mathrm{C} 11-\mathrm{H} 11 \mathrm{~B}$ & 107.5 \\
\hline $\mathrm{C} 4-\mathrm{N} 1-\mathrm{N} 2-\mathrm{N} 3$ & $-173.9(4)$ & $\mathrm{C} 207-\mathrm{C} 208-\mathrm{C} 209-\mathrm{C} 210$ & $2.8(12)$ \\
\hline $\mathrm{Ir} 1-\mathrm{N} 1-\mathrm{N} 2-\mathrm{N} 3$ & $8.1(6)$ & $\mathrm{C} 208-\mathrm{C} 209-\mathrm{C} 210-\mathrm{C} 211$ & $-3.8(13)$ \\
\hline $\mathrm{N} 1-\mathrm{N} 2-\mathrm{N} 3-\mathrm{C} 1$ & $-3.1(7)$ & $\mathrm{C} 209-\mathrm{C} 210-\mathrm{C} 211-\mathrm{C} 212$ & $1.5(13)$ \\
\hline $\mathrm{N} 2-\mathrm{N} 3-\mathrm{C} 1-\mathrm{P} 2$ & $165.7(4)$ & $\mathrm{C} 208-\mathrm{C} 207-\mathrm{C} 212-\mathrm{C} 211$ & $-2.7(11)$ \\
\hline $\mathrm{N} 2-\mathrm{N} 3-\mathrm{C} 1-\mathrm{Ir} 1$ & $-3.0(6)$ & $\mathrm{P} 2-\mathrm{C} 207-\mathrm{C} 212-\mathrm{C} 211$ & $175.1(6)$ \\
\hline $\mathrm{C} 207-\mathrm{P} 2-\mathrm{C} 1-\mathrm{N} 3$ & $-47.9(5)$ & $\mathrm{C} 210-\mathrm{C} 211-\mathrm{C} 212-\mathrm{C} 207$ & $1.7(12)$ \\
\hline
\end{tabular}




$\begin{array}{ll}\text { C2-P2-C1-N3 } & -168.5(4) \\ \text { C201-P2-C1-N3 } & 73.7(5) \\ \text { C207-P2-C1-Ir1 } & 120.0(4) \\ \text { C2-P2-C1-Ir1 } & -0.6(4) \\ \text { C201-P2-C1-Ir1 } & -118.4(3) \\ \text { C1-P2-C2-P1 } & 22.3(4) \\ \text { C207-P2-C2-P1 } & -97.7(3) \\ \text { C201-P2-C2-P1 } & 145.3(3) \\ \text { C107-P1-C2-P2 } & 88.6(3) \\ \text { C101-P1-C2-P2 } & -165.8(3) \\ \text { Ir1-P1-C2-P2 } & -33.4(3) \\ \text { C401-P4-C3-P3 } & -150.9(3) \\ \text { C407-P4-C3-P3 } & 99.3(3) \\ \text { Ir1-P4-C3-P3 } & -18.4(3) \\ \text { C301-P3-C3-P4 } & 143.3(3) \\ \text { C307-P3-C3-P4 } & -109.4(3) \\ \text { Ir1-P3-C3-P4 } & 17.4(2) \\ \text { N2-N1-C4-C5 } & 110.7(6) \\ \text { Ir1-N1-C4-C5 } & -71.5(7) \\ \text { N2-N1-C4-C9 } & -69.1(7) \\ \text { Ir1-N1-C4-C9 } & 108.6(6) \\ \text { C9-C4-C5-C6 } & -0.7(10) \\ \text { N1-C4-C5-C6 } & 179.4(6) \\ \text { C4-C5-C6-C7 } & -0.7(10) \\ \text { C5-C6-C7-C } & 2.0(11) \\ \text { C5-C6-C7-C12 } & -175.9(5) \\ \text { C6-C7-C8-C9 } & -1.9(10) \\ \text { C12-C7-C8-C9 } & 176.0(5) \\ \text { C5-C4-C9-C8 } & 0.8(9) \\ \text { N1-C4-C9-C } & -179.3(5) \\ \text { C7-C8-C9-C4 } & 0.5(10) \\ \text { C107-P1-C101-C106 } & 128.3(6) \\ \text { C2-P1-C101-C106 } & 22.1(6) \\ \text { Ir1-P1-C101-C106 } & -98.8(6) \\ \text { C107-P1-C101-C102 } & -55.4(5) \\ \text { C2-P1-C101-C102 } & -161.7(5) \\ \text { Ir1-P1-C101-C102 } & 77.5(5) \\ \text { C106-C101-C102-C103 } & 2.0(10) \\ \text { P1-C101-C102-C103 } & -174.4(6) \\ \text { C101-C102-C103-C104 } & -1.1(12) \\ \text { C102-C103-C104-C105 } & -1.0(14) \\ \text { C103-C104-C105-C106 } & 2.2(14) \\ \text { C102-C101-C106-C105 } & -0.8(11) \\ \text { P1-C101-C106-C105 } & 175.4(6) \\ \text { C104-C105-C106-C101 } & -1.3(13) \\ \text { C101-P1-C107-C108 } & 145.3(6) \\ \text { C2-P1-C107-C108 } & -108.9(6) \\ \text { Ir1-P1-C107-C108 } & 7.2(6) \\ & \end{array}$

$\begin{array}{ll}\text { C307-P3-C301-C302 } & 105.5(6) \\ \text { C3-P3-C301-C302 } & -145.5(6) \\ \text { Ir1-P3-C301-C302 } & -38.6(7) \\ \text { P4-P3-C301-C302 } & -107.2(6) \\ \text { C307-P3-C301-C306 } & -72.4(6) \\ \text { C3-P3-C301-C306 } & 36.6(6) \\ \text { Ir1-P3-C301-C306 } & 143.5(5) \\ \text { P4-P3-C301-C306 } & 74.9(7) \\ \text { C306-C301-C302-C303 } & -0.9(12) \\ \text { P3-C301-C302-C303 } & -178.8(7) \\ \text { C301-C302-C303-C304 } & 0.7(15) \\ \text { C302-C303-C304-C305 } & 0.3(17) \\ \text { C303-C304-C305-C306 } & -1.1(17) \\ \text { C304-C305-C306-C301 } & 0.8(14) \\ \text { C302-C301-C306-C305 } & 0.1(11) \\ \text { P3-C301-C306-C305 } & 178.1(6) \\ \text { C301-P3-C307-C312 } & 150.3(7) \\ \text { C3-P3-C307-C312 } & 37.6(7) \\ \text { Ir1-P3-C307-C312 } & -66.7(7) \\ \text { P4-P3-C307-C312 } & -6.9(7) \\ \text { C301-P3-C307-C308 } & -28.8(6) \\ \text { C3-P3-C307-C308 } & -141.4(6) \\ \text { Ir1-P3-C307-C308 } & 114.3(5) \\ \text { P4-P3-C307-C308 } & 174.1(5) \\ \text { C312-C307-C308-C309 } & -1.7(11) \\ \text { P3-C307-C308-C309 } & 177.4(6) \\ \text { C307-C308-C309-C310 } & 2.0(13) \\ \text { C308-C309-C310-C311 } & -1.6(15) \\ \text { C309-C310-C311-C312 } & 1.0(16) \\ \text { C308-C307-C312-C311 } & 1.0(13) \\ \text { P3-C307-C312-C311 } & -178.0(8) \\ \text { C310-C311-C312-C307 } & -0.7(16) \\ \text { C407-P4-C401-C406 } & 106.9(6) \\ \text { C3-P4-C401-C406 } & -7.0(7) \\ \text { Ir1-P4-C401-C406 } & -122.2(6) \\ \text { P3-P4-C401-C406 } & -41.4(8) \\ \text { C407-P4-C401-C402 } & -71.2(5) \\ \text { C3-P4-C401-C402 } & 175.0(5) \\ \text { Ir1-P4-C401-C402 } & 59.8(6) \\ \text { P3-P4-C401-C402 } & 140.6(4) \\ \text { C406-C401-C402-C403 } & 0.9(10) \\ \text { P4-C401-C402-C403 } & 179.0(6) \\ \text { C401-C402-C403-C404 } & 0.7(12) \\ \text { C402-C403-C404-C405 } & -1.7(14) \\ \text { C403-C404-C405-C406 } & 1.0(15) \\ \text { C402-C401-C406-C405 } & -1.6(11) \\ \text { P4-C401-C406-C405 } & -179.6(6) \\ \text { C404-C405-C406-C401 } & 0.7(13) \\ & \end{array}$




$\begin{array}{ll}\mathrm{C} 101-\mathrm{P} 1-\mathrm{C} 107-\mathrm{C} 112 & -39.5(6) \\ \mathrm{C} 2-\mathrm{P} 1-\mathrm{C} 107-\mathrm{C} 112 & 66.3(6) \\ \mathrm{I} 1-\mathrm{P} 1-\mathrm{C} 107-\mathrm{C} 112 & -177.6(5) \\ \mathrm{C} 112-\mathrm{C} 107-\mathrm{C} 108-\mathrm{C} 109 & 0.8(11) \\ \mathrm{P} 1-\mathrm{C} 107-\mathrm{C} 108-\mathrm{C} 109 & 176.1(7) \\ \mathrm{C} 107-\mathrm{C} 108-\mathrm{C} 109-\mathrm{C} 110 & -0.8(15) \\ \mathrm{C} 108-\mathrm{C} 109-\mathrm{C} 110-\mathrm{C} 111 & -0.2(17) \\ \mathrm{C} 109-\mathrm{C} 110-\mathrm{C} 111-\mathrm{C} 112 & 1.2(16) \\ \mathrm{C} 110-\mathrm{C} 111-\mathrm{C} 112-\mathrm{C} 107 & -1.2(13) \\ \mathrm{C} 108-\mathrm{C} 107-\mathrm{C} 112-\mathrm{C} 111 & 0.2(11) \\ \mathrm{P} 1-\mathrm{C} 107-\mathrm{C} 112-\mathrm{C} 111 & -175.1(6) \\ \mathrm{C} 1-\mathrm{P} 2-\mathrm{C} 201-\mathrm{C} 202 & -14.3(6) \\ \mathrm{C} 207-\mathrm{P} 2-\mathrm{C} 201-\mathrm{C} 202 & 109.8(6) \\ \mathrm{C} 2-\mathrm{P} 2-\mathrm{C} 201-\mathrm{C} 202 & -129.6(5) \\ \mathrm{C} 1-\mathrm{P} 2-\mathrm{C} 201-\mathrm{C} 206 & 164.5(5) \\ \mathrm{C} 207-\mathrm{P} 2-\mathrm{C} 201-\mathrm{C} 206 & -71.4(6) \\ \mathrm{C} 2-\mathrm{P} 2-\mathrm{C} 201-\mathrm{C} 206 & 49.2(6) \\ \mathrm{C} 206-\mathrm{C} 201-\mathrm{C} 202-\mathrm{C} 203 & 1.1(10) \\ \mathrm{P} 2-\mathrm{C} 201-\mathrm{C} 202-\mathrm{C} 203 & 179.8(6) \\ \mathrm{C} 201-\mathrm{C} 202-\mathrm{C} 203-\mathrm{C} 204 & -0.5(12) \\ \mathrm{C} 202-\mathrm{C} 203-\mathrm{C} 204-\mathrm{C} 205 & -0.5(13) \\ \mathrm{C} 203-\mathrm{C} 204-\mathrm{C} 205-\mathrm{C} 206 & 0.8(13) \\ \mathrm{C} 204-\mathrm{C} 205-\mathrm{C} 206-\mathrm{C} 201 & -0.2(12) \\ \mathrm{C} 202-\mathrm{C} 201-\mathrm{C} 206-\mathrm{C} 205 & -0.8(10) \\ \mathrm{P} 2-\mathrm{C} 201-\mathrm{C} 206-\mathrm{C} 205 & -179.6(6) \\ \mathrm{C} 1-\mathrm{P} 2-\mathrm{C} 207-\mathrm{C} 208 & 74.4(6) \\ \mathrm{C} 2-\mathrm{P} 2-\mathrm{C} 207-\mathrm{C} 208 & -170.3(5) \\ \mathrm{C} 201-\mathrm{P} 2-\mathrm{C} 207-\mathrm{C} 208 & -52.4(6) \\ \mathrm{C} 1-\mathrm{P} 2-\mathrm{C} 207-\mathrm{C} 212 & -103.4(6) \\ \mathrm{C} 2-\mathrm{P} 2-\mathrm{C} 207-\mathrm{C} 212 & 11.9(7) \\ \mathrm{C} 201-\mathrm{P} 2-\mathrm{C} 207-\mathrm{C} 212 & 129.8(6) \\ \mathrm{C} 212-\mathrm{C} 207-\mathrm{C} 208-\mathrm{C} 209 & 0.5(11) \\ \mathrm{P} 2-\mathrm{C} 207-\mathrm{C} 208-\mathrm{C} 209 & -177.4(6) \\ & \end{array}$

$\begin{array}{ll}\text { C401-P4-C407-C408 } & 123.5(5) \\ \text { C3-P4-C407-C408 } & -121.7(5) \\ \text { Ir1-P4-C407-C408 } & -14.8(6) \\ \text { P3-P4-C407-C408 } & -75.7(5) \\ \text { C401-P4-C407-C412 } & -58.2(6) \\ \text { C3-P4-C407-C412 } & 56.7(6) \\ \text { Ir1-P4-C407-C412 } & 163.6(4) \\ \text { P3-P4-C407-C412 } & 102.6(5) \\ \text { C412-C407-C408-C409 } & -2.7(10) \\ \text { P4-C407-C408-C409 } & 175.7(5) \\ \text { C407-C408-C409-C410 } & 0.5(11) \\ \text { C408-C409-C410-C411 } & 2.0(12) \\ \text { C409-C410-C411-C412 } & -2.3(12) \\ \text { C410-C411-C412-C407 } & 0.1(11) \\ \text { C408-C407-C412-C411 } & 2.4(10) \\ \text { P4-C407-C412-C411 } & -175.9(5) \\ \text { C17-C12-C13-C14 } & -11(10) \\ \text { C18-C12-C13-C14 } & -175(7) \\ \text { C12-C13-C14-C15 } & -4(10) \\ \text { C13-C14-C15-C16 } & 17(9) \\ \text { C14-C15-C16-C17 } & -16(8) \\ \text { C15-C16-C17-C12 } & 1(12) \\ \text { C13-C12-C17-C16 } & 13(12) \\ \text { C18-C12-C17-C16 } & 177(8) \\ \text { C24-C19-C20-C21 } & 14(9) \\ \text { C25-C19-C20-C21 } & -175(6) \\ \text { C19-C20-C21-C22 } & 1(9) \\ \text { C20-C21-C22-C23 } & -11(8) \\ \text { C21-C22-C23-C24 } & 4(9) \\ \text { C22-C23-C24-C19 } & 12(9) \\ \text { C20-C19-C24-C23 } & -20(9) \\ \text { C25-C19-C24-C23 } & 168(6) \\ & \end{array}$

Hydrogen-bond geometry $\left(\AA,{ }^{\circ}\right)$

\begin{tabular}{lllll}
\hline$D-\mathrm{H} \cdots A$ & $D-\mathrm{H}$ & $\mathrm{H} \cdots A$ & $D \cdots A$ & $D-\mathrm{H} \cdots A$ \\
\hline $\mathrm{C} 2-\mathrm{H} 2 B \cdots \mathrm{C} 11$ & 0.98 & 2.62 & $3.5215(1)$ & 153 \\
$\mathrm{C} 11-\mathrm{H} 11 B^{\cdots} \cdots \mathrm{C} 11^{\mathrm{i}}$ & 0.98 & 2.49 & $3.4594(1)$ & 170 \\
$\mathrm{C} 209-\mathrm{H} 209 \cdots \mathrm{N} 3^{\mathrm{ii}}$ & 0.94 & 2.60 & $3.3722(1)$ & 140 \\
$\mathrm{C} 306-\mathrm{H} 306 \cdots \mathrm{C} 11 A^{\mathrm{iii}}$ & 0.94 & 2.81 & $3.7037(1)$ & 159 \\
\hline
\end{tabular}

Symmetry codes: (i) $-x+1 / 2, y+1 / 2, z$; (ii) $-x+1, y,-z-1 / 2$; (iii) $-x+1 / 2,-y+3 / 2, z+1 / 2$. 
\{4-[3-(4-Chlorophenyl)triazenido- $\left.\kappa N^{3}\right]$-1,1,3,3-tetraphenyl-1,3 $\lambda^{5}$-diphospha- $\kappa P^{1}$-but-2-en-4-

yl\}cyanido[methylenebis(diphenylphosphine) $-\kappa^{2} P, P^{\prime}$ ]iridium(III) methanol disolvate (3)

Crystal data

$\left[\operatorname{Ir}(\mathrm{CN})\left(\mathrm{C}_{23} \mathrm{H}_{22} \mathrm{P}_{2}\right)\left(\mathrm{C}_{34} \mathrm{H}_{26} \mathrm{ClN}_{3} \mathrm{P}_{2}\right)\right] \cdot 2 \mathrm{CH}_{4} \mathrm{O}$

$M_{r}=1216.61$

Triclinic, $P \overline{1}$

$a=11.1683(1) \AA$

$b=12.7805(2) \AA$

$c=20.0591(3) \AA$

$\alpha=98.475(1)^{\circ}$

$\beta=93.122(1)^{\circ}$

$\gamma=109.336(1)^{\circ}$

$V=2655.75(6) \AA^{3}$

Data collection

Nonius KappaCCD

diffractometer

phi- and $\omega$-scans

20072 measured reflections

10396 independent reflections

9895 reflections with $I>2 \sigma(I)$

Refinement

Refinement on $F^{2}$

Least-squares matrix: full

$R\left[F^{2}>2 \sigma\left(F^{2}\right)\right]=0.023$

$w R\left(F^{2}\right)=0.056$

$S=1.05$

10396 reflections

661 parameters

0 restraints
$Z=2$
$F(000)=1228$
$D_{\mathrm{x}}=1.521 \mathrm{Mg} \mathrm{m}^{-3}$

Mo $K \alpha$ radiation, $\lambda=0.71073 \AA$

Cell parameters from 31262 reflections

$\theta=1.0-27.5^{\circ}$

$\mu=2.73 \mathrm{~mm}^{-1}$

$T=223 \mathrm{~K}$

Prism, colourless

$0.31 \times 0.30 \times 0.12 \mathrm{~mm}$

Special details

Experimental. All data sets were measured with several scans to increase the number of redundant reflections. In our experience this method of averaging redundant reflections replaces in a good approximation semi-empirical absorption methods (absorption correction programs like SORTAV lead to no better data sets).

Geometry. All esds (except the esd in the dihedral angle between two 1.s. planes) are estimated using the full covariance matrix. The cell esds are taken into account individually in the estimation of esds in distances, angles and torsion angles; correlations between esds in cell parameters are only used when they are defined by crystal symmetry. An approximate (isotropic) treatment of cell esds is used for estimating esds involving l.s. planes.

Refinement. Hydrogen atoms at $\mathrm{C} 1$ and $\mathrm{C} 2$ were localized and refined with isotropic displacement parameters.

Fractional atomic coordinates and isotropic or equivalent isotropic displacement parameters $\left(\AA^{2}\right)$

\begin{tabular}{lllll}
\hline & $x$ & $y$ & $z$ & $U_{\text {iso }} * U_{\text {eq }}$ \\
\hline Ir1 & $0.35118(2)$ & $0.22849(2)$ & $0.74840(2)$ & $0.01977(4)$ \\
C11 & $-0.33530(7)$ & $-0.10915(7)$ & $0.67497(5)$ & $0.0557(2)$ \\
P1 & $0.37841(6)$ & $0.34073(5)$ & $0.85641(3)$ & $0.02148(13)$ \\
P2 & $0.46189(6)$ & $0.51310(5)$ & $0.77072(3)$ & $0.02315(13)$ \\
P3 & $0.36652(6)$ & $0.12853(6)$ & $0.64246(3)$ & $0.02637(14)$ \\
P4 & $0.54330(6)$ & $0.19402(5)$ & $0.75590(3)$ & $0.02464(14)$
\end{tabular}




\begin{tabular}{|c|c|c|c|c|}
\hline $\mathrm{N} 1$ & $0.17849(19)$ & $0.24596(18)$ & $0.71335(11)$ & $0.0256(5)$ \\
\hline $\mathrm{N} 2$ & $0.1886(2)$ & $0.31929(18)$ & $0.67036(11)$ & $0.0294(5)$ \\
\hline N3 & $0.2975(2)$ & $0.38693(18)$ & $0.66488(11)$ & $0.0298(5)$ \\
\hline $\mathrm{C} 1$ & $0.4080(2)$ & $0.3808(2)$ & $0.70859(13)$ & $0.0238(5)$ \\
\hline H1 & $0.473(3)$ & $0.388(3)$ & $0.6813(16)$ & $0.040(8)^{*}$ \\
\hline $\mathrm{C} 2$ & $0.4452(2)$ & $0.4807(2)$ & $0.84909(14)$ & $0.0280(6)$ \\
\hline $\mathrm{H} 2$ & $0.455(3)$ & $0.539(2)$ & $0.8856(15)$ & $0.033(8)^{*}$ \\
\hline $\mathrm{C} 3$ & $0.5057(3)$ & $0.0974(2)$ & $0.67372(14)$ & $0.0319(6)$ \\
\hline $\mathrm{H} 3 \mathrm{~A}$ & 0.5750 & 0.1169 & 0.6448 & $0.038^{*}$ \\
\hline H3B & 0.4840 & 0.0185 & 0.6791 & $0.038^{*}$ \\
\hline $\mathrm{C} 4$ & $0.0537(2)$ & $0.1642(2)$ & $0.70456(14)$ & $0.0273(5)$ \\
\hline $\mathrm{C} 5$ & $0.0021(2)$ & $0.1141(2)$ & $0.75831(14)$ & $0.0310(6)$ \\
\hline H5 & 0.0487 & 0.1373 & 0.8015 & $0.037^{*}$ \\
\hline C6 & $-0.1164(3)$ & $0.0310(2)$ & $0.74920(16)$ & $0.0359(6)$ \\
\hline H6 & -0.1502 & -0.0022 & 0.7860 & $0.043^{*}$ \\
\hline $\mathrm{C} 7$ & $-0.1853(3)$ & $-0.0034(2)$ & $0.68569(17)$ & $0.0384(7)$ \\
\hline $\mathrm{C} 8$ & $-0.1381(3)$ & $0.0446(3)$ & $0.63178(16)$ & $0.0403(7)$ \\
\hline $\mathrm{H} 8$ & -0.1864 & 0.0212 & 0.5890 & $0.048^{*}$ \\
\hline C9 & $-0.0181(3)$ & $0.1284(2)$ & $0.64053(15)$ & $0.0359(6)$ \\
\hline H9 & 0.0149 & 0.1611 & 0.6034 & $0.043^{*}$ \\
\hline C101 & $0.2366(2)$ & $0.3316(2)$ & $0.90152(13)$ & $0.0260(5)$ \\
\hline $\mathrm{C} 102$ & $0.1406(2)$ & $0.3617(2)$ & $0.87082(14)$ & $0.0319(6)$ \\
\hline $\mathrm{H} 102$ & 0.1458 & 0.3780 & 0.8267 & $0.038^{*}$ \\
\hline C103 & $0.0374(3)$ & $0.3679(3)$ & $0.90492(16)$ & $0.0404(7)$ \\
\hline $\mathrm{H} 103$ & -0.0265 & 0.3886 & 0.8838 & $0.048^{*}$ \\
\hline $\mathrm{C} 104$ & $0.0282(3)$ & $0.3438(3)$ & $0.96972(18)$ & $0.0487(8)$ \\
\hline $\mathrm{H} 104$ & -0.0413 & 0.3486 & 0.9928 & $0.058^{*}$ \\
\hline C105 & $0.1213(3)$ & $0.3129(3)$ & $1.00026(17)$ & $0.0511(8)$ \\
\hline H105 & 0.1146 & 0.2956 & 1.0442 & $0.061^{*}$ \\
\hline C106 & $0.2251(3)$ & $0.3070(3)$ & $0.96686(15)$ & $0.0381(7)$ \\
\hline H106 & 0.2883 & 0.2861 & 0.9885 & $0.046^{*}$ \\
\hline $\mathrm{C} 107$ & $0.4795(2)$ & $0.3081(2)$ & $0.91953(13)$ & $0.0261(5)$ \\
\hline $\mathrm{C} 108$ & $0.5897(3)$ & $0.3915(2)$ & $0.95340(14)$ & $0.0333(6)$ \\
\hline H108 & 0.6125 & 0.4648 & 0.9435 & $0.040^{*}$ \\
\hline C109 & $0.6661(3)$ & $0.3677(3)$ & $1.00168(15)$ & $0.0425(7)$ \\
\hline H109 & 0.7408 & 0.4245 & 1.0238 & $0.051^{*}$ \\
\hline $\mathrm{C} 110$ & $0.6325(3)$ & $0.2609(3)$ & $1.01710(16)$ & $0.0462(8)$ \\
\hline H110 & 0.6839 & 0.2447 & 1.0499 & $0.055^{*}$ \\
\hline C111 & $0.5228(3)$ & $0.1775(3)$ & $0.98416(15)$ & $0.0405(7)$ \\
\hline H111 & 0.4995 & 0.1048 & 0.9950 & $0.049^{*}$ \\
\hline $\mathrm{C} 112$ & $0.4471(3)$ & $0.2003(2)$ & $0.93538(14)$ & $0.0321(6)$ \\
\hline H112 & 0.3734 & 0.1427 & 0.9128 & $0.038^{*}$ \\
\hline C201 & $0.3767(2)$ & $0.6072(2)$ & $0.75281(14)$ & $0.0266(5)$ \\
\hline C202 & $0.2929(3)$ & $0.6294(2)$ & $0.79717(16)$ & $0.0365(6)$ \\
\hline H202 & 0.2773 & 0.5932 & 0.8350 & $0.044^{*}$ \\
\hline C203 & $0.2323(3)$ & $0.7053(3)$ & 0.78555 (19) & $0.0472(8)$ \\
\hline H203 & 0.1770 & 0.7213 & 0.8160 & $0.057^{*}$ \\
\hline C204 & $0.2527(3)$ & $0.7568(3)$ & $0.7300(2)$ & $0.0506(9)$ \\
\hline
\end{tabular}




\begin{tabular}{|c|c|c|c|c|}
\hline H204 & 0.2104 & 0.8071 & 0.7222 & $0.061 *$ \\
\hline C205 & 0.3354 (3) & $0.7352(3)$ & $0.68530(19)$ & $0.0482(8)$ \\
\hline H205 & 0.3487 & 0.7703 & 0.6470 & $0.058 *$ \\
\hline C206 & $0.3984(3)$ & $0.6617(2)$ & $0.69712(16)$ & $0.0370(7)$ \\
\hline H206 & 0.4561 & 0.6485 & 0.6674 & $0.044 *$ \\
\hline C207 & $0.6230(2)$ & $0.5994(2)$ & $0.75804(15)$ & $0.0315(6)$ \\
\hline C208 & $0.6933(3)$ & $0.6839(2)$ & $0.81107(18)$ & $0.0424(7)$ \\
\hline H208 & 0.6584 & 0.6933 & 0.8522 & $0.051^{*}$ \\
\hline C209 & $0.8150(3)$ & $0.7547(3)$ & $0.8039(2)$ & $0.0545(10)$ \\
\hline H209 & 0.8617 & 0.8120 & 0.8401 & $0.065^{*}$ \\
\hline $\mathrm{C} 210$ & $0.8674(3)$ & $0.7416(3)$ & $0.7443(2)$ & $0.0600(11)$ \\
\hline $\mathrm{H} 210$ & 0.9498 & 0.7896 & 0.7397 & $0.072^{*}$ \\
\hline C211 & $0.7991(3)$ & $0.6580(3)$ & $0.6913(2)$ & $0.0559(9)$ \\
\hline $\mathrm{H} 211$ & 0.8356 & 0.6485 & 0.6507 & $0.067^{*}$ \\
\hline $\mathrm{C} 212$ & $0.6765(3)$ & $0.5873(3)$ & $0.69726(17)$ & $0.0418(7)$ \\
\hline $\mathrm{H} 212$ & 0.6296 & 0.5315 & 0.6604 & $0.050^{*}$ \\
\hline C301 & $0.2520(3)$ & $-0.0063(2)$ & $0.59993(14)$ & $0.0329(6)$ \\
\hline C302 & 0.1351 (3) & $-0.0519(2)$ & $0.62255(16)$ & $0.0400(7)$ \\
\hline H302 & 0.1138 & -0.0139 & 0.6613 & $0.048^{*}$ \\
\hline C303 & $0.0481(4)$ & $-0.1541(3)$ & $0.58850(19)$ & $0.0555(9)$ \\
\hline H303 & -0.0323 & -0.1836 & 0.6038 & $0.067^{*}$ \\
\hline C304 & $0.0787(4)$ & $-0.2118(3)$ & 0.53297 (19) & $0.0611(10)$ \\
\hline H304 & 0.0193 & -0.2802 & 0.5098 & $0.073^{*}$ \\
\hline C305 & 0.1971 (4) & $-0.1687(3)$ & $0.51126(19)$ & $0.0647(11)$ \\
\hline H305 & 0.2195 & -0.2094 & 0.4740 & $0.078^{*}$ \\
\hline C306 & $0.2839(4)$ & $-0.0658(3)$ & $0.54393(17)$ & $0.0510(8)$ \\
\hline H306 & 0.3640 & -0.0364 & 0.5282 & $0.061^{*}$ \\
\hline C307 & $0.4113(3)$ & $0.2083(2)$ & $0.57471(14)$ & $0.0356(6)$ \\
\hline C308 & $0.3161(4)$ & $0.2147(3)$ & $0.52929(16)$ & $0.0523(9)$ \\
\hline H308 & 0.2305 & 0.1703 & 0.5305 & $0.063^{*}$ \\
\hline C309 & $0.3469(5)$ & $0.2860(4)$ & 0.48258 (19) & $0.0697(12)$ \\
\hline H309 & 0.2820 & 0.2906 & 0.4525 & $0.084^{*}$ \\
\hline $\mathrm{C} 310$ & $0.4706(6)$ & $0.3496(4)$ & $0.4798(2)$ & $0.0771(14)$ \\
\hline H310 & 0.4903 & 0.3979 & 0.4477 & $0.092 *$ \\
\hline C311 & $0.5669(5)$ & $0.3443(4)$ & $0.5230(2)$ & $0.0754(13)$ \\
\hline H311 & 0.6522 & 0.3879 & 0.5204 & $0.091^{*}$ \\
\hline C312 & 0.5367 (4) & $0.2732(3)$ & $0.57100(19)$ & $0.0544(9)$ \\
\hline H312 & 0.6022 & 0.2694 & 0.6010 & $0.065^{*}$ \\
\hline $\mathrm{C} 401$ & $0.5894(2)$ & $0.1128(2)$ & $0.81428(13)$ & $0.0273(5)$ \\
\hline $\mathrm{C} 402$ & $0.5127(3)$ & $0.0026(2)$ & $0.81453(15)$ & $0.0346(6)$ \\
\hline $\mathrm{H} 402$ & 0.4341 & -0.0282 & 0.7871 & $0.041^{*}$ \\
\hline C403 & $0.5503(3)$ & $-0.0625(3)$ & $0.85466(17)$ & $0.0419(7)$ \\
\hline H403 & 0.4980 & -0.1374 & 0.8541 & $0.050^{*}$ \\
\hline $\mathrm{C} 404$ & $0.6653(3)$ & $-0.0170(3)$ & $0.89567(17)$ & $0.0447(8)$ \\
\hline H404 & 0.6916 & -0.0612 & 0.9228 & $0.054^{*}$ \\
\hline C405 & $0.7413(3)$ & 0.0928 (3) & $0.89676(16)$ & $0.0426(7)$ \\
\hline H405 & 0.8186 & 0.1237 & 0.9253 & $0.051^{*}$ \\
\hline C406 & $0.7048(2)$ & $0.1581(2)$ & $0.85609(15)$ & $0.0335(6)$ \\
\hline
\end{tabular}




$\begin{array}{lllll}\text { H406 } & 0.7577 & 0.2329 & 0.8567 & 0.040^{*} \\ \text { C407 } & 0.6942(2) & 0.3041(2) & 0.75248(15) & 0.0314(6) \\ \text { C408 } & 0.7255(2) & 0.4061(2) & 0.79592(15) & 0.0335(6) \\ \text { H408 } & 0.6652 & 0.4208 & 0.8233 & 0.040^{*} \\ \text { C409 } & 0.8465(3) & 0.4872(3) & 0.79924(19) & 0.0458(8) \\ \text { H409 } & 0.8689 & 0.5557 & 0.8297 & 0.055^{*} \\ \text { C410 } & 0.9334(3) & 0.4660(3) & 0.7573(2) & 0.0532(9) \\ \text { H410 } & 1.0137 & 0.5217 & 0.7581 & 0.064^{*} \\ \text { C411 } & 0.9036(3) & 0.3646(3) & 0.7146(2) & 0.0526(9) \\ \text { H411 } & 0.9636 & 0.3506 & 0.6867 & 0.063^{*} \\ \text { C412 } & 0.7852(3) & 0.2833(3) & 0.71273(18) & 0.0429(7) \\ \text { H412 } & 0.7657 & 0.2131 & 0.6843 & 0.051^{*} \\ \text { N4 } & 0.2110(2) & 0.0011(2) & 0.80197(13) & 0.0404(6) \\ \text { C10 } & 0.2640(2) & 0.0841(2) & 0.78323(13) & 0.0263(5) \\ \text { O2 } & 0.1944(4) & 0.4619(3) & 0.54949(16) & 0.0904(10) \\ \text { H2A } & 0.2429 & 0.4526 & 0.5791 & 0.136^{*} \\ \text { C12 } & 0.1072(5) & 0.5034(4) & 0.5799(3) & 0.0897(16) \\ \text { H12A } & 0.1330 & 0.5260 & 0.6283 & 0.135^{*} \\ \text { H12B } & 0.0234 & 0.4453 & 0.5719 & 0.135^{*} \\ \text { H12C } & 0.1041 & 0.5680 & 0.5608 & 0.135^{*} \\ \text { O1 } & 0.1200(6) & -0.1236(4) & 0.9047(3) & 0.1429(19) \\ \text { H1A } & 0.1484 & -0.0999 & 0.8703 & 0.214^{*} \\ \text { C11 } & 0.0930(7) & -0.0440(5) & 0.9440(3) & 0.120(2) \\ \text { H11A } & 0.0488 & -0.0747 & 0.9808 & 0.180^{*} \\ \text { H11B } & 0.0390 & -0.0157 & 0.9176 & 0.180^{*} \\ \text { H11C } & 0.1716 & 0.0170 & 0.9624 & 0.180^{*} \\ & & & & \end{array}$

Atomic displacement parameters $\left(\AA^{2}\right)$

\begin{tabular}{lllllll}
\hline & $U^{11}$ & $U^{22}$ & $U^{33}$ & $U^{12}$ & $U^{13}$ & $U^{23}$ \\
\hline Ir1 & $0.02111(5)$ & $0.01730(6)$ & $0.02081(6)$ & $0.00605(4)$ & $0.00309(4)$ & $0.00405(4)$ \\
C11 & $0.0292(4)$ & $0.0364(4)$ & $0.0900(7)$ & $0.0023(3)$ & $-0.0012(4)$ & $-0.0010(4)$ \\
P1 & $0.0230(3)$ & $0.0193(3)$ & $0.0215(3)$ & $0.0062(2)$ & $0.0027(2)$ & $0.0037(2)$ \\
P2 & $0.0222(3)$ & $0.0183(3)$ & $0.0288(3)$ & $0.0060(2)$ & $0.0034(2)$ & $0.0059(3)$ \\
P3 & $0.0339(3)$ & $0.0246(3)$ & $0.0225(3)$ & $0.0125(3)$ & $0.0043(3)$ & $0.0038(3)$ \\
P4 & $0.0233(3)$ & $0.0224(3)$ & $0.0292(4)$ & $0.0087(3)$ & $0.0040(3)$ & $0.0053(3)$ \\
N1 & $0.0226(10)$ & $0.0280(11)$ & $0.0267(11)$ & $0.0092(9)$ & $-0.0002(8)$ & $0.0058(9)$ \\
N2 & $0.0331(12)$ & $0.0272(12)$ & $0.0285(12)$ & $0.0120(10)$ & $-0.0019(9)$ & $0.0049(9)$ \\
N3 & $0.0378(12)$ & $0.0251(12)$ & $0.0262(12)$ & $0.0105(10)$ & $0.0011(9)$ & $0.0058(9)$ \\
C1 & $0.0250(12)$ & $0.0229(13)$ & $0.0234(13)$ & $0.0065(10)$ & $0.0068(10)$ & $0.0063(10)$ \\
C2 & $0.0341(14)$ & $0.0190(13)$ & $0.0286(14)$ & $0.0061(10)$ & $0.0046(11)$ & $0.0043(11)$ \\
C3 & $0.0355(14)$ & $0.0324(15)$ & $0.0326(15)$ & $0.0187(12)$ & $0.0060(11)$ & $0.0027(12)$ \\
C4 & $0.0216(12)$ & $0.0269(14)$ & $0.0337(14)$ & $0.0099(10)$ & $0.0014(10)$ & $0.0028(11)$ \\
C5 & $0.0321(13)$ & $0.0269(14)$ & $0.0324(15)$ & $0.0098(11)$ & $0.0024(11)$ & $0.0017(11)$ \\
C6 & $0.0317(14)$ & $0.0300(15)$ & $0.0445(17)$ & $0.0094(12)$ & $0.0092(12)$ & $0.0031(13)$ \\
C7 & $0.0252(13)$ & $0.0257(14)$ & $0.058(2)$ & $0.0056(11)$ & $-0.0009(13)$ & $-0.0025(13)$ \\
C8 & $0.0339(15)$ & $0.0384(17)$ & $0.0422(18)$ & $0.0112(13)$ & $-0.0099(13)$ & $-0.0044(14)$ \\
C9 & $0.0319(14)$ & $0.0399(16)$ & $0.0306(15)$ & $0.0079(12)$ & $-0.0014(11)$ & $0.0026(12)$
\end{tabular}




\begin{tabular}{|c|c|c|c|c|c|c|}
\hline $\mathrm{C} 101$ & $0.0264(12)$ & $0.0198(12)$ & $0.0292(14)$ & $0.0051(10)$ & $0.0062(10)$ & $0.0016(10)$ \\
\hline $\mathrm{C} 102$ & $0.0285(13)$ & $0.0315(15)$ & $0.0317(15)$ & $0.0073(11)$ & $0.0005(11)$ & $0.0016(12)$ \\
\hline C103 & $0.0251(13)$ & $0.0425(17)$ & $0.0504(19)$ & $0.0096(12)$ & $0.0041(12)$ & $0.0036(14)$ \\
\hline C104 & $0.0331(16)$ & $0.057(2)$ & $0.055(2)$ & $0.0131(14)$ & $0.0196(14)$ & $0.0068(17)$ \\
\hline C105 & $0.055(2)$ & $0.066(2)$ & $0.0386(18)$ & $0.0221(17)$ & $0.0223(15)$ & $0.0181(17)$ \\
\hline C106 & $0.0401(15)$ & $0.0479(18)$ & $0.0336(16)$ & $0.0201(14)$ & $0.0127(12)$ & $0.0150(13)$ \\
\hline C107 & $0.0291(12)$ & $0.0287(14)$ & $0.0215(12)$ & $0.0121(11)$ & $0.0015(10)$ & $0.0028(10)$ \\
\hline C108 & $0.0345(14)$ & $0.0343(15)$ & $0.0286(14)$ & $0.0110(12)$ & $-0.0006(11)$ & $0.0015(12)$ \\
\hline C109 & $0.0392(16)$ & $0.0499(19)$ & $0.0328(16)$ & $0.0152(14)$ & $-0.0096(13)$ & $-0.0051(14)$ \\
\hline C110 & $0.0558(19)$ & $0.060(2)$ & $0.0303(16)$ & $0.0327(17)$ & $-0.0058(14)$ & $0.0049(15)$ \\
\hline C111 & $0.0593(19)$ & $0.0426(18)$ & $0.0296(15)$ & $0.0277(15)$ & $0.0075(14)$ & $0.0120(13)$ \\
\hline $\mathrm{C} 112$ & $0.0391(15)$ & $0.0305(15)$ & $0.0287(14)$ & $0.0136(12)$ & $0.0054(11)$ & $0.0073(11)$ \\
\hline C201 & $0.0244(12)$ & $0.0161(12)$ & $0.0360(15)$ & $0.0040(9)$ & $-0.0020(10)$ & $0.0030(10)$ \\
\hline $\mathrm{C} 202$ & $0.0319(14)$ & $0.0294(15)$ & $0.0452(17)$ & $0.0098(12)$ & $0.0014(12)$ & $0.0003(13)$ \\
\hline $\mathrm{C} 203$ & $0.0357(16)$ & $0.0344(17)$ & $0.071(2)$ & $0.0174(13)$ & $-0.0012(15)$ & $-0.0034(16)$ \\
\hline C204 & $0.0395(17)$ & $0.0250(15)$ & $0.085(3)$ & $0.0164(13)$ & $-0.0196(17)$ & $-0.0007(16)$ \\
\hline C205 & 0.0517 (19) & $0.0301(16)$ & $0.062(2)$ & $0.0135(14)$ & $-0.0126(16)$ & $0.0145(15)$ \\
\hline C206 & $0.0376(15)$ & $0.0305(15)$ & $0.0429(17)$ & $0.0114(12)$ & $-0.0004(13)$ & $0.0090(13)$ \\
\hline C207 & $0.0234(12)$ & $0.0283(14)$ & $0.0448(17)$ & $0.0075(11)$ & $0.0012(11)$ & $0.0170(12)$ \\
\hline C208 & $0.0326(15)$ & $0.0324(16)$ & $0.057(2)$ & $0.0040(12)$ & $-0.0038(14)$ & $0.0126(14)$ \\
\hline C209 & $0.0367(17)$ & $0.0373(18)$ & $0.078(3)$ & $-0.0021(14)$ & $-0.0114(17)$ & $0.0171(17)$ \\
\hline $\mathrm{C} 210$ & $0.0294(16)$ & $0.057(2)$ & $0.093(3)$ & $0.0015(15)$ & $0.0052(18)$ & $0.043(2)$ \\
\hline $\mathrm{C} 211$ & $0.0370(17)$ & $0.064(2)$ & $0.074(3)$ & $0.0126(16)$ & $0.0211(17)$ & $0.040(2)$ \\
\hline $\mathrm{C} 212$ & $0.0318(15)$ & $0.0429(18)$ & $0.0527(19)$ & $0.0091(13)$ & $0.0087(13)$ & $0.0223(15)$ \\
\hline C301 & $0.0474(16)$ & $0.0239(14)$ & $0.0274(14)$ & $0.0144(12)$ & $-0.0041(12)$ & $0.0019(11)$ \\
\hline C302 & $0.0468(17)$ & $0.0289(15)$ & $0.0388(17)$ & $0.0082(13)$ & $0.0025(13)$ & $0.0008(13)$ \\
\hline C303 & $0.061(2)$ & $0.0341(18)$ & $0.058(2)$ & $0.0005(15)$ & $-0.0018(17)$ & $0.0047(16)$ \\
\hline C304 & $0.088(3)$ & $0.0293(17)$ & $0.050(2)$ & $0.0053(18)$ & $-0.010(2)$ & $-0.0013(16)$ \\
\hline C305 & $0.104(3)$ & $0.042(2)$ & $0.042(2)$ & $0.029(2)$ & $0.000(2)$ & $-0.0146(16)$ \\
\hline C306 & $0.069(2)$ & $0.0428(19)$ & $0.0401(18)$ & $0.0213(17)$ & $0.0081(16)$ & $-0.0024(15)$ \\
\hline C307 & $0.0569(18)$ & $0.0309(15)$ & $0.0254(14)$ & $0.0215(13)$ & $0.0124(13)$ & $0.0065(12)$ \\
\hline C308 & $0.077(2)$ & $0.056(2)$ & $0.0317(17)$ & $0.0309(19)$ & $0.0040(16)$ & $0.0107(15)$ \\
\hline C309 & $0.118(4)$ & $0.069(3)$ & $0.038(2)$ & $0.048(3)$ & $0.010(2)$ & $0.0218(19)$ \\
\hline C310 & $0.151(5)$ & $0.059(3)$ & $0.040(2)$ & $0.049(3)$ & $0.037(3)$ & $0.0278(19)$ \\
\hline C311 & $0.106(3)$ & $0.058(3)$ & $0.064(3)$ & $0.018(2)$ & $0.047(3)$ & $0.027(2)$ \\
\hline C312 & $0.063(2)$ & $0.052(2)$ & $0.052(2)$ & $0.0183(17)$ & $0.0219(17)$ & $0.0175(17)$ \\
\hline $\mathrm{C} 401$ & $0.0283(13)$ & $0.0273(14)$ & $0.0289(14)$ & $0.0123(11)$ & $0.0049(10)$ & $0.0059(11)$ \\
\hline C402 & $0.0331(14)$ & $0.0269(14)$ & $0.0402(16)$ & $0.0068(11)$ & $-0.0016(12)$ & $0.0053(12)$ \\
\hline $\mathrm{C} 403$ & $0.0465(17)$ & $0.0283(15)$ & 0.0509 (19) & $0.0107(13)$ & $0.0012(14)$ & $0.0136(14)$ \\
\hline $\mathrm{C} 404$ & $0.0505(18)$ & $0.0455(19)$ & $0.0488(19)$ & $0.0249(15)$ & $0.0039(15)$ & $0.0219(15)$ \\
\hline $\mathrm{C} 405$ & $0.0335(15)$ & $0.0462(18)$ & $0.0483(19)$ & $0.0136(13)$ & $-0.0067(13)$ & $0.0134(15)$ \\
\hline C406 & $0.0270(13)$ & $0.0297(15)$ & $0.0423(16)$ & $0.0072(11)$ & $0.0000(11)$ & $0.0089(12)$ \\
\hline $\mathrm{C} 407$ & $0.0239(12)$ & $0.0308(14)$ & $0.0452(17)$ & $0.0118(11)$ & $0.0074(11)$ & $0.0171(12)$ \\
\hline $\mathrm{C} 408$ & $0.0256(13)$ & $0.0300(15)$ & $0.0466(17)$ & $0.0103(11)$ & $0.0015(12)$ & $0.0105(13)$ \\
\hline C409 & $0.0302(15)$ & $0.0337(17)$ & $0.070(2)$ & $0.0068(12)$ & $-0.0053(14)$ & $0.0124(15)$ \\
\hline $\mathrm{C} 410$ & $0.0257(15)$ & $0.0431(19)$ & $0.094(3)$ & $0.0092(13)$ & $0.0066(16)$ & $0.0294(19)$ \\
\hline $\mathrm{C} 411$ & $0.0331(16)$ & $0.053(2)$ & 0.085 & $0.0204(15)$ & $0.0261(16)$ & $0.0310(19)$ \\
\hline C412 & $0.0344(15)$ & $0.0371(17)$ & $0.063(2)$ & $0.0162(13)$ & $0.0164(14)$ & $0.0134(15)$ \\
\hline
\end{tabular}




$\begin{array}{lllllll}\mathrm{N} 4 & 0.0439(14) & 0.0306(13) & 0.0466(15) & 0.0086(11) & 0.0101(12) & 0.0148(12) \\ \mathrm{C} 10 & 0.0292(13) & 0.0269(14) & 0.0247(13) & 0.0118(11) & 0.0030(10) & 0.0052(11) \\ \text { O2 } & 0.118(3) & 0.096(2) & 0.069(2) & 0.041(2) & 0.011(2) & 0.0424(19) \\ \text { C12 } & 0.096(4) & 0.070(3) & 0.096(4) & 0.029(3) & -0.034(3) & 0.009(3) \\ \text { O1 } & 0.227(5) & 0.106(3) & 0.138(4) & 0.076(3) & 0.103(4) & 0.078(3) \\ \text { C11 } & 0.146(6) & 0.107(5) & 0.080(4) & 0.004(4) & 0.039(4) & 0.018(3)\end{array}$

Geometric parameters $\left(\AA,{ }^{o}\right)$

\begin{tabular}{|c|c|c|c|}
\hline Ir1—P1 & $2.3595(6)$ & $\mathrm{C} 205-\mathrm{H} 205$ & 0.9400 \\
\hline Ir1-P3 & $2.3584(7)$ & $\mathrm{C} 206-\mathrm{H} 206$ & 0.9400 \\
\hline Ir1-P4 & $2.3304(6)$ & C207-C208 & $1.387(4)$ \\
\hline Ir1-N1 & $2.1090(19)$ & $\mathrm{C} 207-\mathrm{C} 212$ & $1.397(4)$ \\
\hline $\mathrm{Ir} 1-\mathrm{C} 1$ & $2.127(3)$ & C208-C209 & $1.390(4)$ \\
\hline $\operatorname{Ir} 1-\mathrm{C} 10$ & $2.027(3)$ & $\mathrm{C} 208-\mathrm{H} 208$ & 0.9400 \\
\hline $\mathrm{Cl1}-\mathrm{C} 7$ & $1.747(3)$ & $\mathrm{C} 209-\mathrm{C} 210$ & $1.373(5)$ \\
\hline $\mathrm{P} 1-\mathrm{C} 2$ & $1.727(3)$ & С209-H209 & 0.9400 \\
\hline $\mathrm{P} 1-\mathrm{C} 107$ & $1.834(2)$ & $\mathrm{C} 210-\mathrm{C} 211$ & $1.374(5)$ \\
\hline $\mathrm{P} 1-\mathrm{C} 101$ & $1.846(3)$ & $\mathrm{C} 210-\mathrm{H} 210$ & 0.9400 \\
\hline $\mathrm{P} 2-\mathrm{C} 2$ & $1.688(3)$ & $\mathrm{C} 211-\mathrm{C} 212$ & $1.392(4)$ \\
\hline $\mathrm{P} 2-\mathrm{C} 201$ & $1.824(3)$ & $\mathrm{C} 211-\mathrm{H} 211$ & 0.9400 \\
\hline $\mathrm{P} 2-\mathrm{C} 207$ & $1.827(3)$ & $\mathrm{C} 212-\mathrm{H} 212$ & 0.9400 \\
\hline $\mathrm{P} 2-\mathrm{C} 1$ & $1.843(3)$ & $\mathrm{C} 301-\mathrm{C} 302$ & $1.374(4)$ \\
\hline P3- C307 & $1.811(3)$ & $\mathrm{C} 301-\mathrm{C} 306$ & $1.392(4)$ \\
\hline P3-C301 & $1.823(3)$ & $\mathrm{C} 302-\mathrm{C} 303$ & $1.393(4)$ \\
\hline $\mathrm{P} 3-\mathrm{C} 3$ & $1.829(3)$ & C302-H302 & 0.9400 \\
\hline P4-C407 & $1.815(3)$ & $\mathrm{C} 303-\mathrm{C} 304$ & $1.366(5)$ \\
\hline $\mathrm{P} 4-\mathrm{C} 401$ & $1.833(3)$ & С $303-\mathrm{H} 303$ & 0.9400 \\
\hline $\mathrm{P} 4-\mathrm{C} 3$ & $1.844(3)$ & C304-C305 & $1.376(6)$ \\
\hline $\mathrm{N} 1-\mathrm{N} 2$ & $1.347(3)$ & С $304-\mathrm{H} 304$ & 0.9400 \\
\hline $\mathrm{N} 1-\mathrm{C} 4$ & $1.421(3)$ & $\mathrm{C} 305-\mathrm{C} 306$ & $1.390(5)$ \\
\hline $\mathrm{N} 2-\mathrm{N} 3$ & $1.259(3)$ & $\mathrm{C} 305-\mathrm{H} 305$ & 0.9400 \\
\hline $\mathrm{N} 3-\mathrm{C} 1$ & $1.504(3)$ & С $306-\mathrm{H} 306$ & 0.9400 \\
\hline $\mathrm{C} 1-\mathrm{H} 1$ & $0.92(3)$ & C $307-\mathrm{C} 312$ & $1.384(5)$ \\
\hline $\mathrm{C} 2-\mathrm{H} 2$ & $0.93(3)$ & C $307-\mathrm{C} 308$ & $1.393(4)$ \\
\hline $\mathrm{C} 3-\mathrm{H} 3 \mathrm{~A}$ & 0.9800 & C308-C309 & $1.381(5)$ \\
\hline C $3-\mathrm{H} 3 \mathrm{~B}$ & 0.9800 & С $308-\mathrm{H} 308$ & 0.9400 \\
\hline $\mathrm{C} 4-\mathrm{C} 5$ & $1.390(4)$ & C $309-\mathrm{C} 310$ & $1.360(7)$ \\
\hline $\mathrm{C} 4-\mathrm{C} 9$ & $1.405(4)$ & С309-H309 & 0.9400 \\
\hline $\mathrm{C} 5-\mathrm{C} 6$ & $1.378(4)$ & $\mathrm{C} 310-\mathrm{C} 311$ & $1.370(7)$ \\
\hline $\mathrm{C} 5-\mathrm{H} 5$ & 0.9400 & $\mathrm{C} 310-\mathrm{H} 310$ & 0.9400 \\
\hline $\mathrm{C} 6-\mathrm{C} 7$ & $1.383(4)$ & $\mathrm{C} 311-\mathrm{C} 312$ & $1.399(5)$ \\
\hline $\mathrm{C} 6-\mathrm{H} 6$ & 0.9400 & $\mathrm{C} 311-\mathrm{H} 311$ & 0.9400 \\
\hline $\mathrm{C} 7-\mathrm{C} 8$ & $1.366(5)$ & $\mathrm{C} 312-\mathrm{H} 312$ & 0.9400 \\
\hline $\mathrm{C} 8-\mathrm{C} 9$ & $1.394(4)$ & $\mathrm{C} 401-\mathrm{C} 402$ & $1.387(4)$ \\
\hline $\mathrm{C} 8-\mathrm{H} 8$ & 0.9400 & $\mathrm{C} 401-\mathrm{C} 406$ & $1.394(4)$ \\
\hline C9-H9 & 0.9400 & $\mathrm{C} 402-\mathrm{C} 403$ & $1.382(4)$ \\
\hline C101-C106 & $1.395(4)$ & $\mathrm{C} 402-\mathrm{H} 402$ & 0.9400 \\
\hline
\end{tabular}




\begin{tabular}{|c|c|c|c|}
\hline $\mathrm{C} 101-\mathrm{C} 102$ & $1.395(4)$ & $\mathrm{C} 403-\mathrm{C} 404$ & $1.384(4)$ \\
\hline $\mathrm{C} 102-\mathrm{C} 103$ & $1.390(4)$ & $\mathrm{C} 403-\mathrm{H} 403$ & 0.9400 \\
\hline $\mathrm{C} 102-\mathrm{H} 102$ & 0.9400 & $\mathrm{C} 404-\mathrm{C} 405$ & $1.374(4)$ \\
\hline $\mathrm{C} 103-\mathrm{C} 104$ & $1.381(5)$ & C404-H404 & 0.9400 \\
\hline C103-H103 & 0.9400 & $\mathrm{C} 405-\mathrm{C} 406$ & $1.386(4)$ \\
\hline $\mathrm{C} 104-\mathrm{C} 105$ & $1.373(5)$ & $\mathrm{C} 405-\mathrm{H} 405$ & 0.9400 \\
\hline C104-H104 & 0.9400 & $\mathrm{C} 406-\mathrm{H} 406$ & 0.9400 \\
\hline $\mathrm{C} 105-\mathrm{C} 106$ & $1.388(4)$ & C407-C408 & $1.382(4)$ \\
\hline C105-H105 & 0.9400 & C407-C412 & $1.395(4)$ \\
\hline C106-H106 & 0.9400 & $\mathrm{C} 408-\mathrm{C} 409$ & $1.395(4)$ \\
\hline $\mathrm{C} 107-\mathrm{C} 108$ & $1.393(4)$ & $\mathrm{C} 408-\mathrm{H} 408$ & 0.9400 \\
\hline C107-C112 & $1.394(4)$ & $\mathrm{C} 409-\mathrm{C} 410$ & $1.386(5)$ \\
\hline $\mathrm{C} 108-\mathrm{C} 109$ & $1.389(4)$ & C409-H409 & 0.9400 \\
\hline C108-H108 & 0.9400 & $\mathrm{C} 410-\mathrm{C} 411$ & $1.370(5)$ \\
\hline $\mathrm{C} 109-\mathrm{C} 110$ & $1.378(5)$ & $\mathrm{C} 410-\mathrm{H} 410$ & 0.9400 \\
\hline C109-H109 & 0.9400 & $\mathrm{C} 411-\mathrm{C} 412$ & $1.379(4)$ \\
\hline $\mathrm{C} 110-\mathrm{C} 111$ & $1.383(5)$ & $\mathrm{C} 411-\mathrm{H} 411$ & 0.9400 \\
\hline $\mathrm{C} 110-\mathrm{H} 110$ & 0.9400 & $\mathrm{C} 412-\mathrm{H} 412$ & 0.9400 \\
\hline $\mathrm{C} 111-\mathrm{C} 112$ & $1.383(4)$ & $\mathrm{N} 4-\mathrm{C} 10$ & $1.158(3)$ \\
\hline C111-H111 & 0.9400 & $\mathrm{O} 2-\mathrm{C} 12$ & $1.383(6)$ \\
\hline $\mathrm{C} 112-\mathrm{H} 112$ & 0.9400 & $\mathrm{O} 2-\mathrm{H} 2 \mathrm{~A}$ & 0.8300 \\
\hline $\mathrm{C} 201-\mathrm{C} 206$ & $1.392(4)$ & $\mathrm{C} 12-\mathrm{H} 12 \mathrm{~A}$ & 0.9700 \\
\hline $\mathrm{C} 201-\mathrm{C} 202$ & $1.393(4)$ & $\mathrm{C} 12-\mathrm{H} 12 \mathrm{~B}$ & 0.9700 \\
\hline $\mathrm{C} 202-\mathrm{C} 203$ & $1.392(4)$ & $\mathrm{C} 12-\mathrm{H} 12 \mathrm{C}$ & 0.9700 \\
\hline $\mathrm{C} 202-\mathrm{H} 202$ & 0.9400 & $\mathrm{O} 1-\mathrm{C} 11$ & $1.318(7)$ \\
\hline $\mathrm{C} 203-\mathrm{C} 204$ & $1.367(5)$ & $\mathrm{O} 1-\mathrm{H} 1 \mathrm{~A}$ & 0.8300 \\
\hline $\mathrm{C} 203-\mathrm{H} 203$ & 0.9400 & $\mathrm{C} 11-\mathrm{H} 11 \mathrm{~A}$ & 0.9700 \\
\hline $\mathrm{C} 204-\mathrm{C} 205$ & $1.387(5)$ & $\mathrm{C} 11-\mathrm{H} 11 \mathrm{~B}$ & 0.9700 \\
\hline $\mathrm{C} 204-\mathrm{H} 204$ & 0.9400 & $\mathrm{C} 11-\mathrm{H} 11 \mathrm{C}$ & 0.9700 \\
\hline $\mathrm{C} 205-\mathrm{C} 206$ & $1.385(4)$ & & \\
\hline $\mathrm{C} 10-\mathrm{Ir} 1-\mathrm{N} 1$ & $94.15(9)$ & $\mathrm{C} 201-\mathrm{C} 202-\mathrm{H} 202$ & 120.0 \\
\hline $\mathrm{C} 10-\mathrm{Ir} 1-\mathrm{C} 1$ & $169.38(9)$ & $\mathrm{C} 204-\mathrm{C} 203-\mathrm{C} 202$ & $120.4(3)$ \\
\hline $\mathrm{N} 1-\mathrm{Ir} 1-\mathrm{C} 1$ & $75.31(9)$ & $\mathrm{C} 204-\mathrm{C} 203-\mathrm{H} 203$ & 119.8 \\
\hline $\mathrm{C} 10-\mathrm{Ir} 1-\mathrm{P} 4$ & $89.88(7)$ & $\mathrm{C} 202-\mathrm{C} 203-\mathrm{H} 203$ & 119.8 \\
\hline $\mathrm{N} 1-\mathrm{Ir} 1-\mathrm{P} 4$ & $164.45(6)$ & $\mathrm{C} 203-\mathrm{C} 204-\mathrm{C} 205$ & $120.3(3)$ \\
\hline $\mathrm{C} 1-\mathrm{Ir} 1-\mathrm{P} 4$ & $100.63(7)$ & $\mathrm{C} 203-\mathrm{C} 204-\mathrm{H} 204$ & 119.9 \\
\hline $\mathrm{C} 10-\mathrm{Ir} 1-\mathrm{P} 3$ & $92.00(7)$ & $\mathrm{C} 205-\mathrm{C} 204-\mathrm{H} 204$ & 119.9 \\
\hline $\mathrm{N} 1-\mathrm{Ir} 1-\mathrm{P} 3$ & $92.64(6)$ & $\mathrm{C} 206-\mathrm{C} 205-\mathrm{C} 204$ & $119.9(3)$ \\
\hline $\mathrm{C} 1-\mathrm{Ir} 1-\mathrm{P} 3$ & $89.80(7)$ & $\mathrm{C} 206-\mathrm{C} 205-\mathrm{H} 205$ & 120.1 \\
\hline P4-Ir1-P3 & $72.19(2)$ & $\mathrm{C} 204-\mathrm{C} 205-\mathrm{H} 205$ & 120.1 \\
\hline $\mathrm{C} 10-\mathrm{Ir} 1-\mathrm{P} 1$ & $92.84(7)$ & $\mathrm{C} 205-\mathrm{C} 206-\mathrm{C} 201$ & $120.3(3)$ \\
\hline $\mathrm{N} 1-\mathrm{Ir} 1-\mathrm{P} 1$ & $96.69(6)$ & $\mathrm{C} 205-\mathrm{C} 206-\mathrm{H} 206$ & 119.8 \\
\hline $\mathrm{C} 1-\mathrm{Ir} 1-\mathrm{P} 1$ & $87.23(7)$ & $\mathrm{C} 201-\mathrm{C} 206-\mathrm{H} 206$ & 119.8 \\
\hline P4-Ir1-P1 & $98.11(2)$ & $\mathrm{C} 208-\mathrm{C} 207-\mathrm{C} 212$ & $118.8(3)$ \\
\hline $\mathrm{P} 3-\mathrm{Ir} 1-\mathrm{P} 1$ & $169.15(2)$ & $\mathrm{C} 208-\mathrm{C} 207-\mathrm{P} 2$ & $117.7(2)$ \\
\hline $\mathrm{C} 2-\mathrm{P} 1-\mathrm{C} 107$ & $109.18(12)$ & $\mathrm{C} 212-\mathrm{C} 207-\mathrm{P} 2$ & $123.5(2)$ \\
\hline $\mathrm{C} 2-\mathrm{P} 1-\mathrm{C} 101$ & $105.42(12)$ & C207-C208-C209 & $120.5(3)$ \\
\hline
\end{tabular}




\begin{tabular}{|c|c|}
\hline $\mathrm{C} 107-\mathrm{P} 1-\mathrm{C} 101$ & $100.87(12)$ \\
\hline $\mathrm{C} 2-\mathrm{P} 1-\mathrm{Ir} 1$ & $108.81(10)$ \\
\hline $\mathrm{C} 107-\mathrm{P} 1-\mathrm{Ir} 1$ & $113.21(8)$ \\
\hline $\mathrm{C} 101-\mathrm{P} 1-\mathrm{Ir} 1$ & $118.72(8)$ \\
\hline $\mathrm{C} 2-\mathrm{P} 2-\mathrm{C} 201$ & $112.23(13)$ \\
\hline $\mathrm{C} 2-\mathrm{P} 2-\mathrm{C} 207$ & $115.04(13)$ \\
\hline $\mathrm{C} 201-\mathrm{P} 2-\mathrm{C} 207$ & $99.28(12)$ \\
\hline $\mathrm{C} 2-\mathrm{P} 2-\mathrm{C} 1$ & $108.32(13)$ \\
\hline $\mathrm{C} 201-\mathrm{P} 2-\mathrm{C} 1$ & $111.83(11)$ \\
\hline $\mathrm{C} 207-\mathrm{P} 2-\mathrm{C} 1$ & $110.00(13)$ \\
\hline C307-P3-C301 & $104.16(13)$ \\
\hline $\mathrm{C} 307-\mathrm{P} 3-\mathrm{C} 3$ & $107.86(14)$ \\
\hline $\mathrm{C} 301-\mathrm{P} 3-\mathrm{C} 3$ & $105.55(12)$ \\
\hline C307-P3-Ir1 & $117.31(9)$ \\
\hline $\mathrm{C} 301-\mathrm{P} 3-\mathrm{Ir} 1$ & $125.75(10)$ \\
\hline C3-P3-Ir1 & $93.86(9)$ \\
\hline C407-P4-C401 & $102.00(12)$ \\
\hline $\mathrm{C} 407-\mathrm{P} 4-\mathrm{C} 3$ & $106.82(14)$ \\
\hline $\mathrm{C} 401-\mathrm{P} 4-\mathrm{C} 3$ & $103.06(12)$ \\
\hline $\mathrm{C} 407-\mathrm{P} 4-\mathrm{Ir} 1$ & $121.19(9)$ \\
\hline $\mathrm{C} 401-\mathrm{P} 4-\mathrm{Ir} 1$ & $125.97(9)$ \\
\hline $\mathrm{C} 3-\mathrm{P} 4-\mathrm{Ir} 1$ & $94.38(8)$ \\
\hline $\mathrm{N} 2-\mathrm{N} 1-\mathrm{C} 4$ & $111.0(2)$ \\
\hline $\mathrm{N} 2-\mathrm{N} 1-\mathrm{Ir} 1$ & $115.61(15)$ \\
\hline $\mathrm{C} 4-\mathrm{N} 1-\mathrm{Ir} 1$ & $127.86(17)$ \\
\hline $\mathrm{N} 3-\mathrm{N} 2-\mathrm{N} 1$ & $118.7(2)$ \\
\hline $\mathrm{N} 2-\mathrm{N} 3-\mathrm{C} 1$ & $116.7(2)$ \\
\hline $\mathrm{N} 3-\mathrm{C} 1-\mathrm{P} 2$ & $104.86(16)$ \\
\hline $\mathrm{N} 3-\mathrm{C} 1-\mathrm{Ir} 1$ & $109.85(16)$ \\
\hline $\mathrm{P} 2-\mathrm{C} 1-\mathrm{Ir} 1$ & $116.66(13)$ \\
\hline $\mathrm{N} 3-\mathrm{C} 1-\mathrm{H} 1$ & 105.7 (19) \\
\hline $\mathrm{P} 2-\mathrm{C} 1-\mathrm{H} 1$ & 104.7 (19) \\
\hline $\mathrm{Ir} 1-\mathrm{C} 1-\mathrm{H} 1$ & $114.1(19)$ \\
\hline $\mathrm{P} 2-\mathrm{C} 2-\mathrm{P} 1$ & $118.42(16)$ \\
\hline $\mathrm{P} 2-\mathrm{C} 2-\mathrm{H} 2$ & $118.6(18)$ \\
\hline $\mathrm{P} 1-\mathrm{C} 2-\mathrm{H} 2$ & $121.5(19)$ \\
\hline $\mathrm{P} 3-\mathrm{C} 3-\mathrm{P} 4$ & 97.54 (12) \\
\hline $\mathrm{P} 3-\mathrm{C} 3-\mathrm{H} 3 \mathrm{~A}$ & 112.3 \\
\hline $\mathrm{P} 4-\mathrm{C} 3-\mathrm{H} 3 \mathrm{~A}$ & 112.3 \\
\hline $\mathrm{P} 3-\mathrm{C} 3-\mathrm{H} 3 \mathrm{~B}$ & 112.3 \\
\hline $\mathrm{P} 4-\mathrm{C} 3-\mathrm{H} 3 \mathrm{~B}$ & 112.3 \\
\hline $\mathrm{H} 3 \mathrm{~A}-\mathrm{C} 3-\mathrm{H} 3 \mathrm{~B}$ & 109.9 \\
\hline $\mathrm{C} 5-\mathrm{C} 4-\mathrm{C} 9$ & $118.4(2)$ \\
\hline $\mathrm{C} 5-\mathrm{C} 4-\mathrm{N} 1$ & $120.9(2)$ \\
\hline $\mathrm{C} 9-\mathrm{C} 4-\mathrm{N} 1$ & $120.7(2)$ \\
\hline $\mathrm{C} 6-\mathrm{C} 5-\mathrm{C} 4$ & $121.0(3)$ \\
\hline $\mathrm{C} 6-\mathrm{C} 5-\mathrm{H} 5$ & 119.5 \\
\hline $\mathrm{C} 4-\mathrm{C} 5$ & \\
\hline
\end{tabular}

$\mathrm{C} 107-\mathrm{P} 1-\mathrm{C} 101$

$2-\mathrm{P} 1-\mathrm{Ir} 1$

C107-P1-Ir 1

$\mathrm{C} 2-\mathrm{P} 2-\mathrm{C} 207$

C201-P2-C207

$\mathrm{C} 2-\mathrm{P} 2-\mathrm{C}$

$\mathrm{C} 201-\mathrm{P} 2-\mathrm{C} 1$

$\mathrm{C} 207-\mathrm{P} 2-\mathrm{C} 1$

C307-P3-C301

$\mathrm{C} 307-\mathrm{P} 3-\mathrm{C} 3$

C301-P3- Ir 1

$\mathrm{C} 3-\mathrm{P} 3-\mathrm{Ir} 1$

C407-P4-C401

$\mathrm{C} 407-\mathrm{P} 4-\mathrm{C} 3$

$\mathrm{C} 401-\mathrm{P} 4-\mathrm{C} 3$

C407-P4-Ir1

C401-P4-Ir1

$\mathrm{C} 3-\mathrm{P} 4-\mathrm{Ir} 1$

$\mathrm{N} 2-\mathrm{N} 1-\mathrm{Ir}$

$\mathrm{C} 4-\mathrm{N} 1-\mathrm{Ir} 1$

$\mathrm{N} 3-\mathrm{N} 2-\mathrm{N} 1$

N3- 1 - Ir 1

$\mathrm{P} 2-\mathrm{C} 1-\mathrm{Ir} 1$

$\mathrm{Ir} 1-\mathrm{C} 1-\mathrm{H} 1$

$\mathrm{P} 2-\mathrm{C} 2-\mathrm{P} 1$

$-\mathrm{C} 2-\mathrm{H} 2$

$\mathrm{P} 3-\mathrm{C} 3-\mathrm{P} 4$

$\mathrm{P} 3-\mathrm{C} 3-\mathrm{H} 3 \mathrm{~A}$

P4- $\mathrm{C} 3-\mathrm{H} 3 \mathrm{~B}$

$\mathrm{H} 3 \mathrm{~A}-\mathrm{C} 3-\mathrm{H} 3 \mathrm{~B}$
$100.87(12)$
$113.21(8)$
$118.72(8)$
$112.23(13)$
$115.04(13)$
99.28 (12)
$108.32(13)$
111.83 (11)
$110.00(13)$
$104.16(13)$
105.55 (12)
117.31 (9)
93.86 (9)
$102.00(12)$
$106.82(14)$
103.06 (12)
121.19 (9)
125.97 (9)
$94.38(8)$
$115.61(15)$
$127.86(17)$
116.7 (2)
$104.86(16)$
$109.85(16)$
$116.66(13)$
105.7 (19)
104.7 (19)
114.1 (19)
118.42 (16)
118.6 (18)
97.54 (12)
112.3
112.3
112.3
109.9
118.4 (2)
120.9 (2)
120.7 (2)
119.5
119.5

\begin{tabular}{|c|c|}
\hline $\mathrm{C} 207-\mathrm{C} 208-\mathrm{H} 208$ & 119.7 \\
\hline $\mathrm{C} 209-\mathrm{C} 208-\mathrm{H} 208$ & 119.7 \\
\hline $\mathrm{C} 210-\mathrm{C} 209-\mathrm{C} 208$ & $120.4(3)$ \\
\hline $\mathrm{C} 210-\mathrm{C} 209-\mathrm{H} 209$ & 119.8 \\
\hline C208-C209-H209 & 119.8 \\
\hline $\mathrm{C} 209-\mathrm{C} 210-\mathrm{C} 211$ & $119.8(3)$ \\
\hline $\mathrm{C} 209-\mathrm{C} 210-\mathrm{H} 210$ & 120.1 \\
\hline $\mathrm{C} 211-\mathrm{C} 210-\mathrm{H} 210$ & 120.1 \\
\hline $\mathrm{C} 210-\mathrm{C} 211-\mathrm{C} 212$ & $120.6(4)$ \\
\hline $\mathrm{C} 210-\mathrm{C} 211-\mathrm{H} 211$ & 119.7 \\
\hline $\mathrm{C} 212-\mathrm{C} 211-\mathrm{H} 211$ & 119.7 \\
\hline $\mathrm{C} 211-\mathrm{C} 212-\mathrm{C} 207$ & $119.9(3)$ \\
\hline $\mathrm{C} 211-\mathrm{C} 212-\mathrm{H} 212$ & 120.1 \\
\hline $\mathrm{C} 207-\mathrm{C} 212-\mathrm{H} 212$ & 120.1 \\
\hline C302-C301-C306 & $118.9(3)$ \\
\hline $\mathrm{C} 302-\mathrm{C} 301-\mathrm{P} 3$ & $121.5(2)$ \\
\hline $\mathrm{C} 306-\mathrm{C} 301-\mathrm{P} 3$ & $119.5(2)$ \\
\hline $\mathrm{C} 301-\mathrm{C} 302-\mathrm{C} 303$ & $120.5(3)$ \\
\hline $\mathrm{C} 301-\mathrm{C} 302-\mathrm{H} 302$ & 119.7 \\
\hline $\mathrm{C} 303-\mathrm{C} 302-\mathrm{H} 302$ & 119.7 \\
\hline $\mathrm{C} 304-\mathrm{C} 303-\mathrm{C} 302$ & $120.5(4)$ \\
\hline C $304-\mathrm{C} 303-\mathrm{H} 303$ & 119.7 \\
\hline $\mathrm{C} 302-\mathrm{C} 303-\mathrm{H} 303$ & 119.7 \\
\hline $\mathrm{C} 303-\mathrm{C} 304-\mathrm{C} 305$ & $119.4(3)$ \\
\hline $\mathrm{C} 303-\mathrm{C} 304-\mathrm{H} 304$ & 120.3 \\
\hline $\mathrm{C} 305-\mathrm{C} 304-\mathrm{H} 304$ & 120.3 \\
\hline $\mathrm{C} 304-\mathrm{C} 305-\mathrm{C} 306$ & $120.6(3)$ \\
\hline $\mathrm{C} 304-\mathrm{C} 305-\mathrm{H} 305$ & 119.7 \\
\hline $\mathrm{C} 306-\mathrm{C} 305-\mathrm{H} 305$ & 119.7 \\
\hline $\mathrm{C} 305-\mathrm{C} 306-\mathrm{C} 301$ & $119.9(4)$ \\
\hline $\mathrm{C} 305-\mathrm{C} 306-\mathrm{H} 306$ & 120.1 \\
\hline $\mathrm{C} 301-\mathrm{C} 306-\mathrm{H} 306$ & 120.1 \\
\hline $\mathrm{C} 312-\mathrm{C} 307-\mathrm{C} 308$ & $118.6(3)$ \\
\hline $\mathrm{C} 312-\mathrm{C} 307-\mathrm{P} 3$ & $121.8(2)$ \\
\hline $\mathrm{C} 308-\mathrm{C} 307-\mathrm{P} 3$ & $119.3(3)$ \\
\hline $\mathrm{C} 309-\mathrm{C} 308-\mathrm{C} 307$ & $120.3(4)$ \\
\hline $\mathrm{C} 309-\mathrm{C} 308-\mathrm{H} 308$ & 119.9 \\
\hline C307-C308-H308 & 119.9 \\
\hline $\mathrm{C} 310-\mathrm{C} 309-\mathrm{C} 308$ & $120.5(4)$ \\
\hline C310-C309- & 119.8 \\
\hline С $308-\mathrm{C} 309-\mathrm{H} 309$ & 119.8 \\
\hline $\mathrm{C} 309-\mathrm{C} 310-\mathrm{C} 311$ & $120.9(4)$ \\
\hline $\mathrm{C} 309-\mathrm{C} 310-\mathrm{H} 310$ & 119.6 \\
\hline $\mathrm{C} 311-\mathrm{C} 310-\mathrm{H} 310$ & 119.6 \\
\hline $\mathrm{C} 310-\mathrm{C} 311-\mathrm{C} 312$ & $119.2(4)$ \\
\hline $\mathrm{C} 310-\mathrm{C} 311-\mathrm{H} 311$ & 120.4 \\
\hline $\mathrm{C} 312-\mathrm{C} 311-\mathrm{H} 311$ & 120.4 \\
\hline $\mathrm{C} 307-\mathrm{C} 312-\mathrm{C} 311$ & $120.6(4)$ \\
\hline
\end{tabular}




\begin{tabular}{|c|c|}
\hline $\mathrm{C} 5-\mathrm{C} 6-\mathrm{C} 7$ & $119.7(3)$ \\
\hline $\mathrm{C} 5-\mathrm{C} 6-\mathrm{H} 6$ & 120.1 \\
\hline $\mathrm{C} 7-\mathrm{C} 6-\mathrm{H} 6$ & 120.1 \\
\hline $\mathrm{C} 8-\mathrm{C} 7-\mathrm{C} 6$ & $120.9(3)$ \\
\hline $\mathrm{C} 8-\mathrm{C} 7-\mathrm{Cl1}$ & $120.0(2)$ \\
\hline $\mathrm{C} 6-\mathrm{C} 7-\mathrm{Cl1}$ & $119.1(3)$ \\
\hline $\mathrm{C} 7-\mathrm{C} 8-\mathrm{C} 9$ & $119.7(3)$ \\
\hline $\mathrm{C} 7-\mathrm{C} 8-\mathrm{H} 8$ & 120.1 \\
\hline $\mathrm{C} 9-\mathrm{C} 8-\mathrm{H} 8$ & 120.1 \\
\hline $\mathrm{C} 8-\mathrm{C} 9-\mathrm{C} 4$ & $120.2(3)$ \\
\hline $\mathrm{C} 8-\mathrm{C} 9-\mathrm{H} 9$ & 119.9 \\
\hline $\mathrm{C} 4-\mathrm{C} 9-\mathrm{H} 9$ & 119.9 \\
\hline $\mathrm{C} 106-\mathrm{C} 101-\mathrm{C} 102$ & $118.1(2)$ \\
\hline $\mathrm{C} 106-\mathrm{C} 101-\mathrm{P} 1$ & $124.0(2)$ \\
\hline $\mathrm{C} 102-\mathrm{C} 101-\mathrm{P} 1$ & $117.6(2)$ \\
\hline $\mathrm{C} 103-\mathrm{C} 102-\mathrm{C} 101$ & $120.6(3)$ \\
\hline $\mathrm{C} 103-\mathrm{C} 102-\mathrm{H} 102$ & 119.7 \\
\hline $\mathrm{C} 101-\mathrm{C} 102-\mathrm{H} 102$ & 119.7 \\
\hline $\mathrm{C} 104-\mathrm{C} 103-\mathrm{C} 102$ & $120.3(3)$ \\
\hline $\mathrm{C} 104-\mathrm{C} 103-\mathrm{H} 103$ & 119.8 \\
\hline $\mathrm{C} 102-\mathrm{C} 103-\mathrm{H} 103$ & 119.8 \\
\hline $\mathrm{C} 105-\mathrm{C} 104-\mathrm{C} 103$ & $119.6(3)$ \\
\hline $\mathrm{C} 105-\mathrm{C} 104-\mathrm{H} 104$ & 120.2 \\
\hline $\mathrm{C} 103-\mathrm{C} 104-\mathrm{H} 104$ & 120.2 \\
\hline $\mathrm{C} 104-\mathrm{C} 105-\mathrm{C} 106$ & $120.6(3)$ \\
\hline $\mathrm{C} 104-\mathrm{C} 105-\mathrm{H} 105$ & 119.7 \\
\hline $\mathrm{C} 106-\mathrm{C} 105-\mathrm{H} 105$ & 119.7 \\
\hline $\mathrm{C} 105-\mathrm{C} 106-\mathrm{C} 101$ & $120.7(3)$ \\
\hline $\mathrm{C} 105-\mathrm{C} 106-\mathrm{H} 106$ & 119.6 \\
\hline $\mathrm{C} 101-\mathrm{C} 106-\mathrm{H} 106$ & 119.6 \\
\hline $\mathrm{C} 108-\mathrm{C} 107-\mathrm{C} 112$ & $118.6(2)$ \\
\hline $\mathrm{C} 108-\mathrm{C} 107-\mathrm{P} 1$ & $120.4(2)$ \\
\hline $\mathrm{C} 112-\mathrm{C} 107-\mathrm{P} 1$ & $120.99(19)$ \\
\hline $\mathrm{C} 109-\mathrm{C} 108-\mathrm{C} 107$ & $120.7(3)$ \\
\hline $\mathrm{C} 109-\mathrm{C} 108-\mathrm{H} 108$ & 119.6 \\
\hline $\mathrm{C} 107-\mathrm{C} 108-\mathrm{H} 108$ & 119.6 \\
\hline $\mathrm{C} 110-\mathrm{C} 109-\mathrm{C} 108$ & $120.0(3)$ \\
\hline $\mathrm{C} 110-\mathrm{C} 109-\mathrm{H} 109$ & 120.0 \\
\hline $\mathrm{C} 108-\mathrm{C} 109-\mathrm{H} 109$ & 120.0 \\
\hline $\mathrm{C} 109-\mathrm{C} 110-\mathrm{C} 111$ & 119.8 \\
\hline $\mathrm{C} 109-\mathrm{C} 110-\mathrm{H} 110$ & 120.1 \\
\hline $\mathrm{C} 111-\mathrm{C} 110-\mathrm{H} 110$ & 120.1 \\
\hline $\mathrm{C} 110-\mathrm{C} 111-\mathrm{C} 112$ & $120.5(3)$ \\
\hline $\mathrm{C} 110-\mathrm{C} 111-\mathrm{H} 111$ & 119.8 \\
\hline $\mathrm{C} 112-\mathrm{C} 111-\mathrm{H} 111$ & 119.8 \\
\hline $\mathrm{C} 111-\mathrm{C} 112-\mathrm{C} 107$ & $120.4(3)$ \\
\hline $\mathrm{C} 111-\mathrm{C} 112-\mathrm{H} 112$ & 119.8 \\
\hline $\mathrm{C} 107-\mathrm{C} 112-\mathrm{H} 112$ & 119.8 \\
\hline
\end{tabular}

C307-C312-H312

C311-C312-H312

$\mathrm{C} 402-\mathrm{C} 401-\mathrm{C} 406$

C402-C401-P4

$\mathrm{C} 406-\mathrm{C} 401-\mathrm{P} 4$

$\mathrm{C} 403-\mathrm{C} 402-\mathrm{C} 401$

$\mathrm{C} 403-\mathrm{C} 402-\mathrm{H} 402$

C401-C402-H402

$\mathrm{C} 402-\mathrm{C} 403-\mathrm{C} 404$

$\mathrm{C} 402-\mathrm{C} 403-\mathrm{H} 403$

C404-C403-H403

$\mathrm{C} 405-\mathrm{C} 404-\mathrm{C} 403$

$\mathrm{C} 405-\mathrm{C} 404-\mathrm{H} 404$

C403-C404-H404

$\mathrm{C} 404-\mathrm{C} 405-\mathrm{C} 406$

C404-C405-H405

$\mathrm{C} 406-\mathrm{C} 405-\mathrm{H} 405$

$\mathrm{C} 405-\mathrm{C} 406-\mathrm{C} 401$

$\mathrm{C} 405-\mathrm{C} 406-\mathrm{H} 406$

$\mathrm{C} 401-\mathrm{C} 406-\mathrm{H} 406$

$\mathrm{C} 408-\mathrm{C} 407-\mathrm{C} 412$

C408-C407-P4

C412-C407-P4

C407-C408-C409

C407-C408-H408

$\mathrm{C} 409-\mathrm{C} 408-\mathrm{H} 408$

$\mathrm{C} 410-\mathrm{C} 409-\mathrm{C} 408$

$\mathrm{C} 410-\mathrm{C} 409-\mathrm{H} 409$

$\mathrm{C} 408-\mathrm{C} 409-\mathrm{H} 409$

C411-C410-C409

$\mathrm{C} 411-\mathrm{C} 410-\mathrm{H} 410$

$\mathrm{C} 409-\mathrm{C} 410-\mathrm{H} 410$

$\mathrm{C} 410-\mathrm{C} 411-\mathrm{C} 412$

C410-C411-H411

C412-C411-H411

$\mathrm{C} 411-\mathrm{C} 412-\mathrm{C} 407$

$\mathrm{C} 411-\mathrm{C} 412-\mathrm{H} 412$

C407-C412-H412

$\mathrm{N} 4-\mathrm{C} 10-\mathrm{Ir} 1$

$\mathrm{C} 12-\mathrm{O} 2-\mathrm{H} 2 \mathrm{~A}$

$\mathrm{O} 2-\mathrm{C} 12-\mathrm{H} 12 \mathrm{~A}$

$\mathrm{O} 2-\mathrm{C} 12-\mathrm{H} 12 \mathrm{~B}$

$\mathrm{H} 12 \mathrm{~A}-\mathrm{C} 12-\mathrm{H} 12 \mathrm{~B}$

$\mathrm{O} 2-\mathrm{C} 12-\mathrm{H} 12 \mathrm{C}$

$\mathrm{H} 12 \mathrm{~A}-\mathrm{C} 12-\mathrm{H} 12 \mathrm{C}$

$\mathrm{H} 12 \mathrm{~B}-\mathrm{C} 12-\mathrm{H} 12 \mathrm{C}$

$\mathrm{C} 11-\mathrm{O} 1-\mathrm{H} 1 \mathrm{~A}$

$\mathrm{O} 1-\mathrm{C} 11-\mathrm{H} 11 \mathrm{~A}$
119.7

119.7

119.1 (2)

119.99 (19)

120.9 (2)

120.8 (3)

119.6

119.6

119.7 (3)

120.1

120.1

120.0 (3)

120.0

120.0

120.6 (3)

119.7

119.7

119.8 (3)

120.1

120.1

119.1 (3)

119.3 (2)

121.1 (2)

120.1 (3)

119.9

119.9

119.5 (3)

120.3

120.3

120.8 (3)

119.6

119.6

119.7 (3)

120.2

120.2

120.8 (3)

119.6

119.6

178.1 (2)

109.5

109.5

109.5

109.5

109.5

109.5

109.5

109.5

109.5 


\begin{tabular}{|c|c|c|c|}
\hline $\mathrm{C} 206-\mathrm{C} 201-\mathrm{C} 202$ & $119.1(3)$ & $\mathrm{O} 1-\mathrm{C} 11-\mathrm{H} 11 \mathrm{~B}$ & 109.5 \\
\hline $\mathrm{C} 206-\mathrm{C} 201-\mathrm{P} 2$ & $121.1(2)$ & $\mathrm{H} 11 \mathrm{~A}-\mathrm{C} 11-\mathrm{H} 11 \mathrm{~B}$ & 109.5 \\
\hline $\mathrm{C} 202-\mathrm{C} 201-\mathrm{P} 2$ & $119.7(2)$ & $\mathrm{O} 1-\mathrm{C} 11-\mathrm{H} 11 \mathrm{C}$ & 109.5 \\
\hline $\mathrm{C} 203-\mathrm{C} 202-\mathrm{C} 201$ & $120.0(3)$ & $\mathrm{H} 11 \mathrm{~A}-\mathrm{C} 11-\mathrm{H} 11 \mathrm{C}$ & 109.5 \\
\hline $\mathrm{C} 203-\mathrm{C} 202-\mathrm{H} 202$ & 120.0 & $\mathrm{H} 11 \mathrm{~B}-\mathrm{C} 11-\mathrm{H} 11 \mathrm{C}$ & 109.5 \\
\hline $\mathrm{C} 4-\mathrm{N} 1-\mathrm{N} 2-\mathrm{N} 3$ & $-169.9(2)$ & $\mathrm{C} 203-\mathrm{C} 204-\mathrm{C} 205-\mathrm{C} 206$ & $-0.5(5)$ \\
\hline $\mathrm{Ir} 1-\mathrm{N} 1-\mathrm{N} 2-\mathrm{N} 3$ & $-14.0(3)$ & $\mathrm{C} 204-\mathrm{C} 205-\mathrm{C} 206-\mathrm{C} 201$ & $1.6(4)$ \\
\hline $\mathrm{N} 1-\mathrm{N} 2-\mathrm{N} 3-\mathrm{C} 1$ & $-1.1(3)$ & $\mathrm{C} 202-\mathrm{C} 201-\mathrm{C} 206-\mathrm{C} 205$ & $-1.3(4)$ \\
\hline $\mathrm{N} 2-\mathrm{N} 3-\mathrm{C} 1-\mathrm{P} 2$ & $-111.2(2)$ & $\mathrm{P} 2-\mathrm{C} 201-\mathrm{C} 206-\mathrm{C} 205$ & $-178.3(2)$ \\
\hline $\mathrm{N} 2-\mathrm{N} 3-\mathrm{C} 1-\mathrm{Ir} 1$ & $14.9(3)$ & $\mathrm{C} 2-\mathrm{P} 2-\mathrm{C} 207-\mathrm{C} 208$ & $-38.4(3)$ \\
\hline $\mathrm{C} 2-\mathrm{P} 2-\mathrm{C} 1-\mathrm{N} 3$ & $118.60(17)$ & $\mathrm{C} 201-\mathrm{P} 2-\mathrm{C} 207-\mathrm{C} 208$ & $81.5(2)$ \\
\hline $\mathrm{C} 201-\mathrm{P} 2-\mathrm{C} 1-\mathrm{N} 3$ & $-5.6(2)$ & $\mathrm{C} 1-\mathrm{P} 2-\mathrm{C} 207-\mathrm{C} 208$ & $-161.0(2)$ \\
\hline $\mathrm{C} 207-\mathrm{P} 2-\mathrm{C} 1-\mathrm{N} 3$ & $-114.89(17)$ & $\mathrm{C} 2-\mathrm{P} 2-\mathrm{C} 207-\mathrm{C} 212$ & $144.0(2)$ \\
\hline $\mathrm{C} 2-\mathrm{P} 2-\mathrm{C} 1-\mathrm{Ir} 1$ & $-3.16(17)$ & $\mathrm{C} 201-\mathrm{P} 2-\mathrm{C} 207-\mathrm{C} 212$ & $-96.0(2)$ \\
\hline $\mathrm{C} 201-\mathrm{P} 2-\mathrm{C} 1-\mathrm{Ir} 1$ & $-127.35(14)$ & $\mathrm{C} 1-\mathrm{P} 2-\mathrm{C} 207-\mathrm{C} 212$ & $21.4(3)$ \\
\hline $\mathrm{C} 207-\mathrm{P} 2-\mathrm{C} 1-\mathrm{Ir} 1$ & $123.34(13)$ & $\mathrm{C} 212-\mathrm{C} 207-\mathrm{C} 208-\mathrm{C} 209$ & $-0.4(4)$ \\
\hline $\mathrm{C} 201-\mathrm{P} 2-\mathrm{C} 2-\mathrm{P} 1$ & $120.99(16)$ & $\mathrm{P} 2-\mathrm{C} 207-\mathrm{C} 208-\mathrm{C} 209$ & $-178.0(2)$ \\
\hline $\mathrm{C} 207-\mathrm{P} 2-\mathrm{C} 2-\mathrm{P} 1$ & $-126.49(16)$ & $\mathrm{C} 207-\mathrm{C} 208-\mathrm{C} 209-\mathrm{C} 210$ & $-0.3(5)$ \\
\hline $\mathrm{C} 1-\mathrm{P} 2-\mathrm{C} 2-\mathrm{P} 1$ & $-3.0(2)$ & $\mathrm{C} 208-\mathrm{C} 209-\mathrm{C} 210-\mathrm{C} 211$ & $0.1(5)$ \\
\hline $\mathrm{C} 107-\mathrm{P} 1-\mathrm{C} 2-\mathrm{P} 2$ & $131.02(16)$ & $\mathrm{C} 209-\mathrm{C} 210-\mathrm{C} 211-\mathrm{C} 212$ & $0.8(5)$ \\
\hline $\mathrm{C} 101-\mathrm{P} 1-\mathrm{C} 2-\mathrm{P} 2$ & $-121.34(17)$ & $\mathrm{C} 210-\mathrm{C} 211-\mathrm{C} 212-\mathrm{C} 207$ & $-1.5(5)$ \\
\hline $\mathrm{Ir} 1-\mathrm{P} 1-\mathrm{C} 2-\mathrm{P} 2$ & $7.02(18)$ & $\mathrm{C} 208-\mathrm{C} 207-\mathrm{C} 212-\mathrm{C} 211$ & $1.2(4)$ \\
\hline $\mathrm{C} 307-\mathrm{P} 3-\mathrm{C} 3-\mathrm{P} 4$ & $108.45(14)$ & $\mathrm{P} 2-\mathrm{C} 207-\mathrm{C} 212-\mathrm{C} 211$ & $178.8(2)$ \\
\hline $\mathrm{C} 301-\mathrm{P} 3-\mathrm{C} 3-\mathrm{P} 4$ & $-140.67(13)$ & $\mathrm{C} 307-\mathrm{P} 3-\mathrm{C} 301-\mathrm{C} 302$ & $-129.3(2)$ \\
\hline $\mathrm{Ir} 1-\mathrm{P} 3-\mathrm{C} 3-\mathrm{P} 4$ & $-11.87(12)$ & $\mathrm{C} 3-\mathrm{P} 3-\mathrm{C} 301-\mathrm{C} 302$ & $117.2(2)$ \\
\hline $\mathrm{C} 407-\mathrm{P} 4-\mathrm{C} 3-\mathrm{P} 3$ & $-112.44(13)$ & $\mathrm{Ir} 1-\mathrm{P} 3-\mathrm{C} 301-\mathrm{C} 302$ & $10.6(3)$ \\
\hline $\mathrm{C} 401-\mathrm{P} 4-\mathrm{C} 3-\mathrm{P} 3$ & $140.52(13)$ & $\mathrm{C} 307-\mathrm{P} 3-\mathrm{C} 301-\mathrm{C} 306$ & $51.9(3)$ \\
\hline $\mathrm{Ir} 1-\mathrm{P} 4-\mathrm{C} 3-\mathrm{P} 3$ & $12.02(12)$ & $\mathrm{C} 3-\mathrm{P} 3-\mathrm{C} 301-\mathrm{C} 306$ & $-61.6(3)$ \\
\hline $\mathrm{N} 2-\mathrm{N} 1-\mathrm{C} 4-\mathrm{C} 5$ & $-151.0(2)$ & $\mathrm{Ir} 1-\mathrm{P} 3-\mathrm{C} 301-\mathrm{C} 306$ & $-168.3(2)$ \\
\hline $\mathrm{Ir} 1-\mathrm{N} 1-\mathrm{C} 4-\mathrm{C} 5$ & $56.8(3)$ & $\mathrm{C} 306-\mathrm{C} 301-\mathrm{C} 302-\mathrm{C} 303$ & $-2.4(5)$ \\
\hline $\mathrm{N} 2-\mathrm{N} 1-\mathrm{C} 4-\mathrm{C} 9$ & $31.9(3)$ & $\mathrm{P} 3-\mathrm{C} 301-\mathrm{C} 302-\mathrm{C} 303$ & $178.8(3)$ \\
\hline $\mathrm{Ir} 1-\mathrm{N} 1-\mathrm{C} 4-\mathrm{C} 9$ & $-120.4(2)$ & $\mathrm{C} 301-\mathrm{C} 302-\mathrm{C} 303-\mathrm{C} 304$ & $1.5(5)$ \\
\hline $\mathrm{C} 9-\mathrm{C} 4-\mathrm{C} 5-\mathrm{C} 6$ & $0.3(4)$ & $\mathrm{C} 302-\mathrm{C} 303-\mathrm{C} 304-\mathrm{C} 305$ & $0.8(6)$ \\
\hline $\mathrm{N} 1-\mathrm{C} 4-\mathrm{C} 5-\mathrm{C} 6$ & $-176.9(2)$ & $\mathrm{C} 303-\mathrm{C} 304-\mathrm{C} 305-\mathrm{C} 306$ & $-2.1(6)$ \\
\hline $\mathrm{C} 4-\mathrm{C} 5-\mathrm{C} 6-\mathrm{C} 7$ & $0.0(4)$ & $\mathrm{C} 304-\mathrm{C} 305-\mathrm{C} 306-\mathrm{C} 301$ & $1.2(6)$ \\
\hline $\mathrm{C} 5-\mathrm{C} 6-\mathrm{C} 7-\mathrm{C} 8$ & $-0.6(4)$ & $\mathrm{C} 302-\mathrm{C} 301-\mathrm{C} 306-\mathrm{C} 305$ & $1.0(5)$ \\
\hline $\mathrm{C} 5-\mathrm{C} 6-\mathrm{C} 7-\mathrm{Cl}$ & $179.9(2)$ & $\mathrm{P} 3-\mathrm{C} 301-\mathrm{C} 306-\mathrm{C} 305$ & $179.9(3)$ \\
\hline $\mathrm{C} 6-\mathrm{C} 7-\mathrm{C} 8-\mathrm{C} 9$ & $0.8(5)$ & $\mathrm{C} 301-\mathrm{P} 3-\mathrm{C} 307-\mathrm{C} 312$ & $-137.7(3)$ \\
\hline $\mathrm{C} 11-\mathrm{C} 7-\mathrm{C} 8-\mathrm{C} 9$ & $-179.6(2)$ & $\mathrm{C} 3-\mathrm{P} 3-\mathrm{C} 307-\mathrm{C} 312$ & $-25.8(3)$ \\
\hline $\mathrm{C} 7-\mathrm{C} 8-\mathrm{C} 9-\mathrm{C} 4$ & $-0.5(4)$ & $\mathrm{Ir} 1-\mathrm{P} 3-\mathrm{C} 307-\mathrm{C} 312$ & $78.4(3)$ \\
\hline $\mathrm{C} 5-\mathrm{C} 4-\mathrm{C} 9-\mathrm{C} 8$ & $0.0(4)$ & $\mathrm{C} 301-\mathrm{P} 3-\mathrm{C} 307-\mathrm{C} 308$ & $49.5(3)$ \\
\hline $\mathrm{N} 1-\mathrm{C} 4-\mathrm{C} 9-\mathrm{C} 8$ & $177.2(3)$ & $\mathrm{C} 3-\mathrm{P} 3-\mathrm{C} 307-\mathrm{C} 308$ & $161.3(2)$ \\
\hline $\mathrm{C} 2-\mathrm{P} 1-\mathrm{C} 101-\mathrm{C} 106$ & $-112.8(2)$ & $\mathrm{Ir} 1-\mathrm{P} 3-\mathrm{C} 307-\mathrm{C} 308$ & $-94.4(2)$ \\
\hline $\mathrm{C} 107-\mathrm{P} 1-\mathrm{C} 101-\mathrm{C} 106$ & $0.8(3)$ & C $312-\mathrm{C} 307-\mathrm{C} 308-\mathrm{C} 309$ & $-1.1(5)$ \\
\hline $\mathrm{Ir} 1-\mathrm{P} 1-\mathrm{C} 101-\mathrm{C} 106$ & $125.1(2)$ & P3-C307-C308-C309 & $171.9(3)$ \\
\hline $\mathrm{C} 2-\mathrm{P} 1-\mathrm{C} 101-\mathrm{C} 102$ & $60.8(2)$ & $\mathrm{C} 307-\mathrm{C} 308-\mathrm{C} 309-\mathrm{C} 310$ & $0.8(6)$ \\
\hline $\mathrm{C} 107-\mathrm{P} 1-\mathrm{C} 101-\mathrm{C} 102$ & $174.4(2)$ & $\mathrm{C} 308-\mathrm{C} 309-\mathrm{C} 310-\mathrm{C} 311$ & $0.1(6)$ \\
\hline
\end{tabular}




$\begin{array}{ll}\text { Ir1-P1-C101-C102 } & -61.4(2) \\ \text { C106-C101-C102-C103 } & 0.6(4) \\ \text { P1-C101-C102-C103 } & -173.4(2) \\ \text { C101-C102-C103-C104 } & -0.2(4) \\ \text { C102-C103-C104-C105 } & -0.5(5) \\ \text { C103-C104-C105-C106 } & 0.8(5) \\ \text { C104-C105-C106-C101 } & -0.4(5) \\ \text { C102-C101-C106-C105 } & -0.3(4) \\ \text { P1-C101-C106-C105 } & 173.3(3) \\ \text { C2-P1-C107-C108 } & 0.8(3) \\ \text { C101-P1-C107-C108 } & -109.9(2) \\ \text { Ir1-P1-C107-C108 } & 122.2(2) \\ \text { C2-P1-C107-C112 } & 179.9(2) \\ \text { C101-P1-C107-C112 } & 69.2(2) \\ \text { Ir1-P1-C107-C112 } & -58.7(2) \\ \text { C112-C107-C108-C109 } & 0.4(4) \\ \text { P1-C107-C108-C109 } & 179.4(2) \\ \text { C107-C108-C109-C110 } & -0.8(5) \\ \text { C108-C109-C110-C111 } & 0.3(5) \\ \text { C109-C110-C111-C112 } & 0.6(5) \\ \text { C110-C111-C112-C107 } & -1.0(5) \\ \text { C108-C107-C112-C111 } & 0.5(4) \\ \text { P1-C107-C112-C111 } & -178.6(2) \\ \text { C2-P2-C201-C206 } & 167.1(2) \\ \text { C207-P2-C201-C206 } & 45.1(2) \\ \text { C1-P2-C201-C206 } & -71.0(2) \\ \text { C2-P2-C201-C202 } & -9.8(2) \\ \text { C207-P2-C201-C202 } & -131.8(2) \\ \text { C1-P2-C201-C202 } & 112.1(2) \\ \text { C206-C201-C202-C203 } & -0.1(4) \\ \text { P2-C201-C202-C203 } & 176.9(2) \\ \text { C201-C202-C203-C204 } & 1.2(4) \\ \text { C202-C203-C204-C205 } & -0.9(5) \\ & \end{array}$

\begin{tabular}{|c|c|}
\hline $\mathrm{C} 309-\mathrm{C} 310-\mathrm{C} 311-\mathrm{C} 312$ & $-0.7(6)$ \\
\hline $\mathrm{C} 308-\mathrm{C} 307-\mathrm{C} 312-\mathrm{C} 311$ & $0.5(5)$ \\
\hline $\mathrm{P} 3-\mathrm{C} 307-\mathrm{C} 312-\mathrm{C} 311$ & $-172.4(3)$ \\
\hline $\mathrm{C} 310-\mathrm{C} 311-\mathrm{C} 312-\mathrm{C} 307$ & $0.4(6)$ \\
\hline $\mathrm{C} 407-\mathrm{P} 4-\mathrm{C} 401-\mathrm{C} 402$ & $-159.6(2)$ \\
\hline $\mathrm{C} 3-\mathrm{P} 4-\mathrm{C} 401-\mathrm{C} 402$ & $-49.0(3)$ \\
\hline $\mathrm{Ir} 1-\mathrm{P} 4-\mathrm{C} 401-\mathrm{C} 402$ & $56.4(3)$ \\
\hline $\mathrm{C} 407-\mathrm{P} 4-\mathrm{C} 401-\mathrm{C} 406$ & $16.7(3)$ \\
\hline $\mathrm{C} 3-\mathrm{P} 4-\mathrm{C} 401-\mathrm{C} 406$ & $127.4(2)$ \\
\hline $\mathrm{Ir} 1-\mathrm{P} 4-\mathrm{C} 401-\mathrm{C} 406$ & $-127.2(2)$ \\
\hline $\mathrm{C} 406-\mathrm{C} 401-\mathrm{C} 402-\mathrm{C} 403$ & $-1.1(4)$ \\
\hline $\mathrm{P} 4-\mathrm{C} 401-\mathrm{C} 402-\mathrm{C} 403$ & $175.3(2)$ \\
\hline $\mathrm{C} 401-\mathrm{C} 402-\mathrm{C} 403-\mathrm{C} 404$ & $0.7(5)$ \\
\hline $\mathrm{C} 402-\mathrm{C} 403-\mathrm{C} 404-\mathrm{C} 405$ & $0.5(5)$ \\
\hline $\mathrm{C} 403-\mathrm{C} 404-\mathrm{C} 405-\mathrm{C} 406$ & $-1.2(5)$ \\
\hline $\mathrm{C} 404-\mathrm{C} 405-\mathrm{C} 406-\mathrm{C} 401$ & $0.8(5)$ \\
\hline $\mathrm{C} 402-\mathrm{C} 401-\mathrm{C} 406-\mathrm{C} 405$ & $0.3(4)$ \\
\hline $\mathrm{P} 4-\mathrm{C} 401-\mathrm{C} 406-\mathrm{C} 405$ & $-176.1(2)$ \\
\hline $\mathrm{C} 401-\mathrm{P} 4-\mathrm{C} 407-\mathrm{C} 408$ & $-93.6(2)$ \\
\hline $\mathrm{C} 3-\mathrm{P} 4-\mathrm{C} 407-\mathrm{C} 408$ & $158.6(2)$ \\
\hline $\mathrm{Ir} 1-\mathrm{P} 4-\mathrm{C} 407-\mathrm{C} 408$ & $52.6(3)$ \\
\hline $\mathrm{C} 401-\mathrm{P} 4-\mathrm{C} 407-\mathrm{C} 412$ & $78.8(3)$ \\
\hline $\mathrm{C} 3-\mathrm{P} 4-\mathrm{C} 407-\mathrm{C} 412$ & $-29.0(3)$ \\
\hline $\mathrm{Ir} 1-\mathrm{P} 4-\mathrm{C} 407-\mathrm{C} 412$ & $-135.1(2)$ \\
\hline $\mathrm{C} 412-\mathrm{C} 407-\mathrm{C} 408-\mathrm{C} 409$ & $0.6(4)$ \\
\hline P4-C407-C408-C409 & $173.1(2)$ \\
\hline $\mathrm{C} 407-\mathrm{C} 408-\mathrm{C} 409-\mathrm{C} 410$ & $1.9(5)$ \\
\hline $\mathrm{C} 408-\mathrm{C} 409-\mathrm{C} 410-\mathrm{C} 411$ & $-2.6(5)$ \\
\hline $\mathrm{C} 409-\mathrm{C} 410-\mathrm{C} 411-\mathrm{C} 412$ & $0.8(5)$ \\
\hline $\mathrm{C} 410-\mathrm{C} 411-\mathrm{C} 412-\mathrm{C} 407$ & $1.7(5)$ \\
\hline $\mathrm{C} 408-\mathrm{C} 407-\mathrm{C} 412-\mathrm{C} 411$ & $-2.4(5)$ \\
\hline $\mathrm{P} 4-\mathrm{C} 407-\mathrm{C} 412-\mathrm{C} 411$ & $-174.8(3)$ \\
\hline
\end{tabular}

Hydrogen-bond geometry $\left(\AA,{ }^{\circ}\right)$

\begin{tabular}{lllll}
\hline$D-\mathrm{H} \cdots A$ & $D-\mathrm{H}$ & $\mathrm{H} \cdots A$ & $D \cdots A$ & $D-\mathrm{H} \cdots A$ \\
\hline $\mathrm{O} 1-\mathrm{H} 1 A \cdots \mathrm{N} 4$ & 0.83 & 2.02 & $2.8181(1)$ & 162 \\
$\mathrm{O} 2-\mathrm{H} 2 A \cdots \mathrm{N} 3$ & 0.83 & 2.16 & $2.9486(1)$ & 158 \\
\hline
\end{tabular}

